\title{
Absolutely Continuous Invariant Measures for One- Parameter Families of One-Dimensional Maps
}

\author{
M. V. Jakobson \\ Central Scientific-Research Economic Institute, Smolenskii Boulevard, Moscow G-117, USSR
}

\begin{abstract}
Given a one-parameter family $f_{\lambda}(x)$ of maps of the interval $[0,1]$, we consider the set of parameter values $\lambda$ for which $f_{\lambda}$ has an invariant measure absolutely continuous with respect to Lebesgue measure. We show that this set has positive measure, for two classes of maps: i) $f_{\lambda}(x)=\lambda f(x)$ where $0<\lambda \leqq 4$ and $f(x)$ is a function $C^{3}$-near the quadratic map $x(1-x)$, and ii) $f_{\lambda}(x)=\lambda f(x)$ $(\bmod 1)$ where $f$ is $C^{3}, f(0)=f(1)=0$ and $f$ has a unique nondegenerate critical point in $[0,1]$.
\end{abstract}

\section{Introduction}

Dynamical systems generated by noninvertible maps of an interval into itself have been intensely studied recently. The most widely considered was the family $f_{\lambda}: x \rightarrow \lambda x(1-x), x \in[0,1], 0 \leqq \lambda \leqq 4$.

It is well-known that if $f_{\lambda}$ has an attracting periodic orbit $\bar{\alpha}=\left(\alpha_{1}, \ldots, \alpha_{n}\right)$ then all probabilisitic $f_{\lambda}$-invariant measures are singular with respect to a Lebesgue measure $d x$, and the iterations $f_{\lambda *}^{n} d x$ converge in the weak *-topology to the discrete invariant measure supported by $\bar{\alpha}$.

It is probable (but not proved) that this situation is typical from the topological point of view, i.e. for a general one-parameter family of smooth mappings $f_{\lambda}: I \rightarrow I$, $\lambda \in \Lambda$, there is an open and dense subset $\Lambda_{0}$ of $\Lambda$ such that for $\lambda \in \Lambda_{0}$, the set of limit points for $f_{\lambda *}^{n} d x$ consists of a finite number of measures supported by periodic attracting orbits.

We show in the present paper that this is not so from the metric point of view. Namely we prove for a certain class of one-parameter families $f_{\lambda}$ that the set $\Lambda_{1}=\left\{\lambda: f_{\lambda}\right.$ has an invariant finite measure $\mu_{\lambda}$ absolutely continuous with respect to $\left.d x\left(\mu_{\lambda}<d x\right)\right\}$

has a positive measure in $\Lambda$.

In the classical case $x \rightarrow 4 x(1-x)$ considered by Ulam and von Neumann in [1], the invariant measure $\mu(d x)$ has density $\varrho(x)=\frac{1}{\pi \sqrt{x(1-x)}}$. In [2] Bunimovič 
constructed absolutely continuous measures for the piecewise smooth mappings $x \rightarrow n \sin \pi x(\bmod 1), n \in \mathbb{Z}$. Ruelle in [3] considered $f_{\lambda}: x \rightarrow \lambda x(1-x)$ and proved that an invariant measure $\mu_{\lambda}<d x$ exists for $\lambda=3,678 \ldots$ - chosen in such a way that the third iterate of the critical point, $f_{\lambda}^{3}\left(\frac{1}{2}\right)$, falls into the unstable fixed point $x=1-\frac{1}{\lambda}$. Bowen in [4] found sufficient conditions for the existence of an invariant measure $\mu_{\lambda}<d x$ for $f_{\lambda}(x)=\lambda x(1-x)$, when $\frac{1}{2}$ is a preimage of a periodic unstable point. In [5] it was shown that the cardinality of $\left\{\lambda: f_{\lambda}\right.$ has an invariant measure $\left.\mu_{\lambda}<d x\right\}$ is that of the continuum for the family $x \rightarrow \lambda x(1-x)$ and any $C^{2}$-family $f_{\lambda}$ sufficiently close to $\lambda x(1-x)$. Similar results were obtained by Misiurewicz [6] and Szlenk [7] for a class of mappings with negative Schwarzian derivative. Ognev in [8] proved for $x \rightarrow \lambda x(1-x)$ that if $\frac{1}{2}$ is a preimage of a periodic unstable point, then the density of the invariant measure is analytic. Ito, Tanaka, Nakada in [9] studied the space of parameters of unimodal linear transformations and found explicitly the densities of the invariant measures.

Collet and Eckmann in [10] proved for a particular family $f_{\delta}(x)$ that $f_{\delta}$ has sensitive dependence with respect to initial conditions in the sense of Guckenheimer [11] for a set of $\delta$ of positive measure. The mappings $f_{\gamma}$ obtained with our construction are also sensitive dependent. It is unknown whether sensitive dependence implies existence of absolutely continuous invariant measure.

We shall consider two kinds of one-parameter families $f_{\lambda}(x)$.

1. Piecewise smooth families $x \mapsto \lambda f(x)(\bmod 1)$, where $f(x):[0,1] \rightarrow[0,1]$ is a $C^{3}$-map with a single nondegenerate critical point, $f(0)=f(1)=0$, and $\lambda$ is a big parameter.

2. Smooth families $x \mapsto \lambda x(1-x) 0 \leqq \lambda \leqq 4$, and $\lambda \cdot f(x)$ with $f(x)$ sufficiently close to $x(1-x)$ in $C^{3}([0,1],[0,1])$.

We formulate now our main results.

Theorem A. Let $f_{\lambda}: x \rightarrow \lambda f(x)(\bmod 1)$ be a piecewise smooth family. There exists $T_{0}>0$, such that for any $\varepsilon>0$ there is an $L(\varepsilon)$, so that if $L \geqq L(\varepsilon)$ then the interval $[L$, $\left.L+T_{0}\right]$ on the $\lambda$-axis contains a set $\mathscr{M}$ satisfying

i) $\operatorname{mes} \mathscr{M}>T_{0}-\varepsilon$;

ii) $\forall \lambda \in \mathscr{M} f_{\lambda}$ admits an invariant measure $\mu_{\lambda}<d x$.

Theorem B. Let $f_{\lambda}(x)$ be one of the smooth families mentioned above. Then there is a set of positive measure $\Lambda_{1}$ so that for $\lambda \in \Lambda_{1} f_{\lambda}$ admits an invariant measure $\mu_{\lambda}<d x$.

Remark. The parameter values $\lambda_{1}$ such that the critical point of $f_{\lambda_{1}}$ is contained in the preimage of an unstable periodic orbit (e.g. $\lambda_{1}=4$ for $\lambda \cdot x(1-x)$, or in the preimage of a certain invariant unstable Cantor set (see [5])) turn out to be onesided Lebesgue points of $\Lambda_{1}$, i.e. $\forall \varepsilon>0 \exists \delta>0$, such that

$$
\operatorname{mes}\left\{\lambda \in \Lambda_{1}: \lambda_{1} \geqq \lambda \geqq \lambda_{1}-\delta\right\}>\delta(1-\varepsilon) \text {. }
$$

In Sects. $1-12$ we prove Theorem A for the family $x \rightarrow \lambda x(1-x)(\bmod 1)$. In Sect. 13 we point out modifications concerning the case of an arbitrary family $x \rightarrow \lambda \cdot f(x)$ $(\bmod 1)$ and show how to reduce the proof of Theorem B to the proof of Theorem A. 


\section{Idea of Proof}

The number $T_{0}$ for the family $f_{\lambda}: x \rightarrow \lambda x(1-x)(\bmod 1)$ equals $4:$ as $\lambda$ varies from $L$ to $L+4$, the image of the critical point $f_{\lambda}\left(\frac{1}{2}\right)=\frac{\lambda}{4}(\bmod 1)$ passes over the entire interval $[0,1])$. In order to prove Theorem $A$ we must find for a given $\varepsilon>0$ an $L(\varepsilon)$ such that, if $L \geqq L(\varepsilon)$ then the interval $[L, L+4]$ contains a set $\mathscr{M}$ so that mes $\mathscr{M}>4$ $-\varepsilon$ and for any $\lambda \in \mathscr{M} f_{\lambda}$ has an invariant measure $\mu_{\lambda}<d x$. Without loss of generality we can assume that $\lambda$ varies from $N_{0}=4 k_{0}$ to $N_{0}+4, k_{0} \in \mathbb{Z}_{+}$. For a smooth map $g(\lambda, x)$ we shall use the notation $D g_{\partial} D^{2} g$ for $\frac{\partial g(\lambda, x)}{\partial x}, \frac{\partial^{2} g(\lambda, x)}{\partial x^{2}}$.

The central part of the proof of Theorem $\mathrm{A}$ is the construction for $\lambda \in \mathscr{M}$ of a special partition $\xi_{\lambda}$ of $[0,1]$. The elements of $\xi_{\lambda}$ are intervals $\Delta_{i}(\lambda), i \in \mathbb{Z}_{+}$, which satisfy the following conditions:

i) int $\left.\Delta_{i}(\lambda) \cap \operatorname{int} \Delta_{j}(\lambda)\right)=\emptyset$.

ii) $\forall i \exists n_{i} \in \mathbb{Z}_{+}$such that $f_{\lambda}^{n_{i}}$ maps $\Delta_{i}(\lambda)$ diffeomorphically onto $[0,1]$.

iii) $\inf _{\Delta_{i} \in \xi_{\lambda}} \min _{x \in \Delta_{i}}\left|D f_{\lambda}^{n_{2}}(x)\right|>\lambda^{c_{0}}$ for some $c_{0}>0(\lambda$ is a big parameter here, so $\lambda \gg 0)$.

iv) $\sup _{\Delta_{i} \in \xi_{\lambda}} \max _{x \in \Delta_{i}}\left|\frac{D^{2} f_{\lambda}^{n_{2}}(x)}{D f_{\lambda}^{n_{i}}(x)}\right| \cdot\left|\Delta_{i}(\lambda)\right|<1+\lambda^{-t_{1}}$, for some $t_{1}>0$.

Let $\mathscr{X}(\lambda)$ be the union of all elements $\Delta_{i}(\lambda)$ of $\xi_{\lambda}$. Then $\mathscr{X}(\lambda)=[0,1](\bmod 0)$.

The set $\mathscr{M}$ and the sets $\mathscr{X}(\lambda)$ for $\lambda \in \mathscr{M}$ are constructed by induction. $\mathscr{M}$ is obtained as an intersection $\mathscr{M}=\bigcap_{n=0}^{\infty} \mathscr{M}_{n}$, where

$$
\begin{gathered}
\mathscr{M}_{0}=\left[N_{0}, N_{0}+4\right], \quad \mathscr{M}_{n+1} \subset \mathscr{M}_{n}, \\
\text { mes } \mathscr{M}_{n+1}>\left(1-\varepsilon_{n+1}\right) \operatorname{mes} \mathscr{M}_{n}, \quad \sum_{n=1}^{\infty} \varepsilon_{n}=O\left(\lambda^{-t_{2}}\right), \quad t_{2}>0 .
\end{gathered}
$$

At the $n$th induction step, we define for any $\lambda \in \mathscr{M}_{n-1}$ a set $\mathscr{X}_{n}(\lambda) \subset[0,1]$ which is the union of a countable number of intervals $\Delta_{i}^{(k)}(\lambda), k=1, \ldots, n$. The intervals constructed at step $k$ do not change at the next steps. The sets $\mathscr{X}_{n}(\lambda)$ satisfy the following properties :

$$
\mathscr{X}_{n}(\lambda) \subset \mathscr{X}_{n+1}(\lambda) ; \quad \operatorname{mes} \mathscr{X}_{n}(\lambda)>1-\lambda^{-t_{3} n}, \quad t_{3}>0 .
$$

Finally we set $\mathscr{X}(\lambda)=\bigcup_{n=1}^{\infty} \mathscr{X}_{n}(\lambda)$. Any element $\Delta_{i}(\lambda)$ of $\xi_{\lambda}$ coincides with one of $\Delta_{i}^{(n)}(\lambda)$.

Let us define the map $T_{\lambda}: \mathscr{X}(\lambda) \rightarrow[0,1]$ by $T_{\lambda} \mid \Delta_{i}(\lambda)=f_{\lambda}^{n_{2}}$. The results of Adler [12] and Walters [13] imply the existence and the uniqueness of a $T_{\lambda}$-invariant measure $v_{\lambda}<d x$. The endomorphism $\left([0,1], T_{\lambda}, v_{\lambda}\right)$ is exact, and its natural extension is a Bernoulli shift. The $f_{\lambda}$-invariant measure $\mu_{\lambda}$ is constructed from $v_{\lambda}$.

\section{First Steps of the Inductive Construction}

The graph of the map $f_{\lambda}$ consists of a lot of monotone branches which we denote by $f(\lambda, x)$ and the middle parabola denoted by $h(\lambda, x)$. The domains of $f(\lambda, x)$ and 
$h(\lambda, x)$ depend continuously on $\lambda$. When $\lambda=4 k_{0}$, a new middle branch is born, which exists for $\lambda \leqq 4\left(k_{0}+1\right)$ and then breaks up into two monotone branches.

We shall denote by $\Delta f(\lambda, x)$ the domain of $f(\lambda, x)$, by $x_{\min }(\lambda)$ the endpoint nearest to $\frac{1}{2}$ of the interval $\Delta f(\lambda, x)$, and by $x_{\max }(\lambda)$ the other endpoint of $\Delta f(\lambda, x)$. We shall distinguish $[a, b]$ from $[b, a]$ according to its position relative to $\frac{1}{2}$ and not according to its orientation.

We fix a positive number $s<\frac{1}{13}$.

\section{Step 1}

Pick the branch $f^{\prime}(\lambda, x)$ of $f_{\lambda}$ whose domain $\Delta f^{\prime}(\lambda, x)=\Delta^{\prime}(\lambda)=\left[x_{\min }^{\prime}(\lambda), x_{\max }^{\prime}(\lambda)\right]$ is contained in $\left[0, \frac{1}{2}\right]$ and is closest to $\frac{1}{2}$, subject to the condition

$$
\left|x_{\min }^{\prime}(\lambda)-\frac{1}{2}\right|>\lambda^{-s} \text { for all } \lambda \in \mathscr{M}_{0} \text {. }
$$

Denote by $\Delta^{\prime \prime}(\lambda)=\Delta f^{\prime \prime}(\lambda, x)$ the analogous interval in $\left[\frac{1}{2}, 1\right]$. Define $\delta_{1}(\lambda)=\left[x_{\min }^{\prime}(\lambda)\right.$, $\left.x_{\min }^{\prime \prime}(\lambda)\right]$, noting that $\delta_{1}(\lambda)$ has the form

$$
\delta_{1}(\lambda)=\left[\frac{1}{2}-r_{1}(\lambda), \frac{1}{2}+r_{1}(\lambda)\right], \quad r_{1}(\lambda)>\lambda^{-s}
$$

and let $\mathscr{X}_{1}(\lambda)=[0,1] \backslash \delta_{1}(\lambda)$. Thus,

$$
[0,1]=\mathscr{X}_{1}(\lambda) \bigcup \delta_{1}(\lambda) \text {. }
$$

Both $\mathscr{X}_{1}(\lambda)$ and $\delta_{1}(\lambda)$ are the union of several domains of branches, $\Delta f(\lambda, x)$, varying continuously with $\lambda$.

Since

$$
|D f(\lambda, x)|=2 \lambda\left|x-\frac{1}{2}\right|,
$$

we have

$$
\left|\Delta^{\prime}(\lambda)\right|<\frac{1}{2} \lambda^{-1+s}
$$

and

$$
\left|\frac{d x_{\min }^{\prime}(\lambda)}{d \lambda}\right|=\left|\frac{\partial f(\lambda, x) / \partial \lambda}{\partial f(\lambda, x) / \partial x}\right|_{x=x_{\min }^{\prime}(\lambda)}<\frac{1}{8 \lambda^{1-s}}
$$

This implies

$$
\frac{1}{\lambda^{s}}<r_{1}(\lambda)<\frac{1}{\lambda^{s}}+\frac{1}{\lambda^{1-s}}=\frac{1}{\lambda^{s}}\left[1+\frac{1}{\lambda^{1-2 s}}\right] .
$$

In order to construct the set $\mathscr{M}_{1}$ we consider the domains $\Delta f(\lambda, x)=\left[x_{\min }(\lambda)\right.$, $\left.x_{\max }(\lambda)\right]$ satisfying

$$
\left|x_{\min }(\lambda)-\frac{1}{2}\right|>1 / \lambda^{s / 2}
$$

We obtain as above that for any such domain

$$
\begin{aligned}
& |\Delta f(\lambda, x)|<\frac{1}{2} \lambda^{-1+s / 2} \\
& \left|\frac{d x_{\min }(\lambda)}{d \lambda}\right|<\frac{1}{8} \lambda^{-1+s / 2} .
\end{aligned}
$$


The top of the graph, $h\left(\lambda, \frac{1}{2}\right)$, moves with velocity

$$
\frac{d h\left(\lambda, \frac{1}{2}\right)}{d \lambda}=\frac{1}{4}
$$

A comparison of velocities shows that to each branch $f_{i}(\lambda, x)$ with domain $\Delta_{i}(\lambda)$ there corresponds a uniquely defined interval $\mathscr{J}_{i}=\mathscr{J}\left(\Delta_{i}\right)$ of $\lambda$-values such that, as $\lambda$ ranges over $\mathscr{J}_{i}$, the top $h\left(\lambda, \frac{1}{2}\right)$ ranges over $\Delta_{i}(\lambda)$ and its image $f_{i}\left(\lambda, h\left(\lambda, \frac{1}{2}\right)\right)$ ranges over $[0,1]$.

So we define $\mathscr{M}_{1}$ as the union of these $\mathscr{J}_{i}$ :

$$
\mathscr{M}_{1}=\bigcup\left\{\mathscr{J}_{i}=\mathscr{J}\left(\Delta_{i}\right)\left|\left(\forall \lambda \in \mathscr{M}_{0}\right)\right| x_{\min }(\lambda)-\frac{1}{2} \mid>1 / \lambda^{s / 2}\right\} .
$$

It follows from the estimates (2.1), (2.1a) and (2.1b) that

$$
\operatorname{mes} \mathscr{M}_{1}>4\left[1-\max _{N_{0} \leqq \lambda \leqq N_{0}+4} \operatorname{mes} \mathscr{X}_{1}(\lambda)\right]>4\left[1-\frac{2\left(1+\gamma_{1}\right)}{\mathrm{N}_{0}^{s / 2}}\right] \text {, }
$$

where

$$
\gamma_{1}<1 / N_{0}^{1-s}
$$

Step 2. Construction of $\mathscr{X}_{2}(\lambda)$

Let us denote by $f_{1}$ the branches $f(\lambda, x)$ such that $\Delta f \subset \mathscr{X}_{1}(\lambda)$ and by $g$ the branches with $\Delta g \subset \delta_{1}(\lambda)$. Let us consider compositions $f_{1}^{\circ} g$. Any domain $\Delta g$ can be represented in the form

$$
\Delta g=\bigcup \Delta\left(f_{1} \circ g\right) \cup \bigcup g^{-1}\left(\delta_{1}\right)
$$

Choose an interval

$$
\delta_{2}(\lambda)=\left[\frac{1}{2}-\frac{c_{21}}{\lambda^{2 s}}, \frac{1}{2}+\frac{c_{22}}{\lambda^{2 s}}\right], \quad 1<c_{21}, c_{22}<1+O\left(1 / \lambda^{1-3 s}\right)
$$

which is a union of domains $\Delta\left(f_{1} \circ g\right)$ and $g^{-1} \delta_{1}$. We shall use $g_{1}$ to denote $g \mid \delta_{1} \backslash \delta_{2}$ and $f_{21}$ to denote $f_{1} \circ g_{1}$. Then (2.3) implies

$$
\delta_{1}=\bigcup \Delta f_{21} \cup \bigcup g_{1}^{-1} \delta_{1} \cup \delta_{2} .
$$

For any particular branch $\tilde{g}_{1}$ we have

$$
\tilde{g}_{1}^{-1}\left(\delta_{1}\right)=\bigcup \tilde{g}_{1}^{-1}\left(\Delta f_{21}\right) \cup \bigcup \tilde{g}_{1}^{-1} \circ g_{1}^{-1}\left(\delta_{1}\right) \cup \tilde{g}_{1}^{-1} \delta_{2},
$$

where the large unions are over all $f_{21}$ and $g_{1}$ respectively. Denote the branches $f_{21} \circ g_{1}$ by $f_{22}$. Since $\Delta\left(f_{21} \circ g_{1}\right)=g_{1}^{-1}\left(\Delta f_{21}\right)$, we can rewrite $(2.4)$ as

$$
\delta_{1}=\bigcup \Delta f_{21} \cup \bigcup \Delta f_{22} \cup \bigcup g_{1}^{-2}\left(\delta_{1}\right) \cup \bigcup g_{1}^{-1}\left(\delta_{2}\right) \cup \delta_{2},
$$

where $g_{1}^{-2}$ denotes any composition of the form $\tilde{g}_{1}^{-1} \circ \tilde{g}_{1}^{-1}$. Proceeding in the same way we obtain the representation

$$
\begin{aligned}
\delta_{1}= & \cup \Delta f_{21} \cup \bigcup \Delta f_{22} \cup \ldots \cup \bigcup \Delta f_{2 k} \cup \bigcup g_{1}^{-(k-1)}\left(\delta_{2}\right) \\
& \cdot \cup \ldots \cup \bigcup g_{1}^{-1}\left(\delta_{2}\right) \cup \delta_{2} \cup \bigcup g_{1}^{-k} \delta_{1}
\end{aligned}
$$


where

$$
\begin{gathered}
f_{2 \ell}=f_{21} \circ g_{1 i_{1}} \circ \ldots \circ g_{1 i_{\ell-1}} \\
g_{1}^{-r}=g_{1 i_{r}}^{-1} \circ \ldots \circ g_{1 i_{1}}^{-1} .
\end{gathered}
$$

Any branch $g_{1}$ satisfies

$$
\begin{gathered}
\left|D g_{1}\right|>2 \lambda^{1-2 s} \\
\left|D^{2} g_{1}\right|=2 \lambda
\end{gathered}
$$

from which it follows (see for example [11]) that

$$
\lim _{k \rightarrow \infty} \operatorname{mes}\left[\bigcup g_{1}^{-k}\left(\delta_{1}\right)\right]=0 \text {. }
$$

Therefore, we can write

$$
\delta_{1}=\bigcup_{k=1}^{\infty}\left(\Delta f_{2 k}\right) \cup \bigcup_{k=1}^{\infty} g_{1}^{-k}\left(\delta_{2}\right) \cup \delta_{2}(\bmod 0)
$$

where mod 0 means we neglect sets with zero Lebesgue measure. (Hereafter, in analogous equalities, "mod 0 " will be understood.) Using the notation $f_{2}$ for all the $f_{2 k}, k=1,2, \ldots$, we obtain

$$
[0,1]=\bigcup \Delta f_{1} \cup \bigcup \Delta f_{2} \cup \bigcup_{k=1}^{\infty} g_{1}^{-k}\left(\delta_{2}\right) \cup \delta_{2}
$$

or

$$
[0,1]=\mathscr{X}_{2}(\lambda) \cup \bigcup_{k=1}^{\infty} g_{1}^{-k}\left(\delta_{2}\right) \cup \delta_{2},
$$

where by construction $\mathscr{X}_{2}(\lambda)$ is partitioned by the various domains $\Delta f_{1}$ and $\Delta f_{2}$ constructed in steps 1 and 2 . These domains will be elements of the partition $\xi_{\lambda}$.

Now (2.3) and (2.8) induce an analogous structure inside $\delta_{2}$ :

$$
\delta_{2}=\bigcup \Delta\left(f_{1} \circ g\right) \cup \bigcup_{k=1}^{\infty} \Delta\left(f_{2 k} \circ g\right) \cup \bigcup_{n=0}^{\infty} g^{-1} \circ g_{1}^{-n}\left(\delta_{2}\right) \text {. }
$$

Notice that one of the $g$ 's in (2.11) stands for $h$. Suppose $h\left(\frac{1}{2}\right) \in \Delta \tilde{f}_{1}$. Then for any other branch $f_{1} \neq \tilde{f}_{1}$ either $f_{1}$ oh has two monotone branches or none; similarly $h^{-1}$ on $\delta_{2}$ has two or no monotone branches. The only branch of parabolic type in (2.11) is $\tilde{f}_{1} \circ h$.

We see from $(2.10)$ that $\mathscr{X}_{2}(\lambda)$ is the complement (mod 0$)$ of the preimages of $\delta_{2}$ under the various branches $g_{1}^{k}(k \geqq 0)$. At the end of the next section, we will see that $\mathscr{M}_{2}$ is the set of those $\lambda \in \mathscr{M}_{1}$ for which the appropriate branch $f_{1}$ takes the critical value $h\left(\lambda, \frac{1}{2}\right)$ into the complement of the $g_{1}^{k}$-preimages of an interval $\hat{\delta}_{2}$ which is also small but much larger than $\delta_{2}$.

\section{Step $n+1$. Geometrical Part}

We assume after step $n$ that the set $\mathscr{M}_{n}$ has been defined and for every $\lambda \in \mathscr{M}_{n}$ the set $\mathscr{X}_{n}(\lambda)$ has been constructed. Every $\mathscr{X}_{n}(\lambda)$ is a countable union of domains $\Delta f_{k}(\lambda, x)$, 
$k=1,2, \ldots, n$, where we use $f_{k}$ to denote a branch constructed at step $k$. The interval $[0,1]$ can be represented $(\bmod 0)$ in the following form:

$$
[0,1]=\left[\bigcup_{k=1}^{n}\left(\bigcup \Delta f_{k}\right)\right] \cup\left[\bigcup_{m=1}^{\infty}\left(\bigcup \delta_{n}^{-m}\right)\right] \cup \delta_{n} .
$$

Here the interval

$$
\delta_{n}=\delta_{n}(\lambda)=\left[\frac{1}{2}-\frac{c_{n 1}}{\lambda^{s n}}, \frac{1}{2}+\frac{c_{n 2}}{\lambda^{s n}}\right], \quad 1 \leqq c_{n 1}, c_{n 2} \leqq 1+0\left(\frac{1}{\lambda^{t n}}\right), \quad t=\frac{\alpha}{10},
$$

and $\delta_{n}^{-m}$ are various diffeomorphic preimages of $\delta_{n}$. We shall denote by $G_{n}: \delta_{n}^{-m} \rightarrow \delta_{n}$ the corresponding diffeomorphisms without pointing out their dependence on $m$; if $m=0, G_{n}=$ Id.

In order to describe the representation of $\delta_{n}$ analogous to (3.1) we need some additional notation. Let $F_{n-1}$ be a composition of maps $f_{k}$ constructed at the previous steps:

$$
F_{n-1}=f_{i_{n-1}} \circ f_{i_{n-2}} \circ \ldots \circ f_{i_{2}} \circ f_{i_{1}}, \quad i_{1}=1, \quad i_{2} \in[1,2], \ldots, i_{n-1} \in[1, n-1] .
$$

We shall distinguish two kinds of branches for various powers of $f$ with domains inside $\delta_{n}$ : the first have the form $F_{n-1} \circ g(\lambda, x)\left(F_{n-1} \circ h(\lambda, x)\right.$ for the central branch) where $g$ denotes the initial map $x \rightarrow \lambda x(1-x)$ : and the second kind are all the remaining branches, mapping their domains diffeomorphically onto $[0,1]$, and denoted by $\hat{f}_{n}(\lambda, x)$. So we assume $\delta_{n}$ has the following representation after Step $n$ :

$$
\delta_{n}=\left(\bigcup \Delta F_{n-1} \circ g\right) \cup\left(\bigcup \Delta \hat{f}_{n}\right) \cup\left[\bigcup_{m=m_{n}}^{\infty}\left(\bigcup \delta_{n}^{-m}\right)\right] .
$$

Now for any $\lambda \in \mathscr{M}_{n}$ we describe the construction of $\mathscr{X}_{n+1}(\lambda)$. The estimates which allow us to realize this construction are adduced in subsequent sections.

a) We consider the compositions $f_{k} \circ F_{n-1} \circ g$ and $f_{k} \circ \hat{f}_{n}$ for all $f_{k}(k \in[1, n])$, $F_{n-1} \circ \mathrm{g}$, and $\hat{f}_{n}$. Then the domains $\Delta F_{n-1} \circ g$ and $\Delta \hat{f}_{n}$ have the following representations

$$
\left.\begin{array}{rl}
\Delta F_{n-1} \circ g & =\left[\bigcup_{k=1}^{n}\left(\bigcup \Delta f_{k} \circ F_{n-1} \circ g\right)\right] \cup\left[\bigcup_{m=0}^{\infty}\left(\bigcup\left(F_{n-1} \circ g\right)^{-1}\left(\delta_{n}^{-m}\right)\right)\right] \\
\Delta \hat{f}_{n} & =\left[\bigcup_{k=1}^{n}\left(\bigcup \Delta f_{k} \circ \hat{f}_{n}\right)\right] \cup\left[\bigcup_{m=0}^{\infty}\left(\bigcup \hat{f}_{n}^{-1}\left(\delta_{n}^{-m}\right)\right)\right]
\end{array}\right\} .
$$

Notice that the representation (3.3) for $\Delta F_{n-1} \circ$ contains only the members corresponding to $\Delta f_{k}$ and $\delta_{n}^{-m}$ which lie in the image of $F_{n-1} \circ h$.

b) In (3.3) some new preimages of $\delta_{n}$ arose, namely $\left(F_{n-1} \circ g\right)^{-1} \delta_{n}^{-m}$ and $\hat{f}_{n}^{-1} \delta_{n}^{-m}$. We still denote them $\delta_{n}^{-m}$, but the corresponding diffeomorphisms $G_{n} \circ F_{n-1} \circ g$ and $G_{n} \circ \hat{f}_{n}$ will be denoted by $G_{n}^{\prime}$. Let us rewrite (3.3) in the form

$$
\left.\begin{array}{rl}
\Delta F_{n-1} \circ g & =\left(\bigcup \Delta f_{k} \circ F_{n-1} \circ g\right) \cup\left(\bigcup \delta_{n}^{-m}\right) \\
\Delta \hat{f}_{n} & =\left(\bigcup \Delta f_{k} \circ \hat{f}_{n}\right) \cup\left(\bigcup \delta_{n}^{-m}\right)
\end{array}\right\} .
$$


Now we choose an interval $\delta_{n+1}(\lambda)$ composed of whole elements of the partition generated in (3.2) and (3.4):

$$
\delta_{n+1}(\lambda)=\left[\frac{1}{2}-\frac{c_{n+1,1}(\lambda)}{\lambda^{s(n+1)}}, \frac{1}{2}+\frac{c_{n+1,2}(\lambda)}{\lambda^{s(n+1)}}\right], \quad 1 \leqq c_{n+1, i} \leqq 1+0\left(\frac{1}{\lambda^{t(n+1)}}\right)
$$

c) We shall distinguish the maps with domains in $\delta_{n} \backslash \delta_{n+1}$, thus we use some additional notation.

Let $g_{n}=\lambda x(1-x)(\bmod 1) \mid \delta_{n} \backslash \delta_{n+1}$. We shall use $f_{n+11}$ to denote the branches $f_{k} \circ F_{n-1} \circ g_{n}$ and $f_{k} \circ \hat{f}_{n} \mid \delta_{n} \backslash \delta_{n+1}$. Finally, we shall use $\tilde{G}_{n}$ to denote the $G_{n}$ or $G_{n}^{\prime}$ with domain inside $\delta_{n} \backslash \delta_{n+1}$. Using (3.2) and (3.4) we obtain the following representation of $\delta_{n}$ :

$$
\delta_{n}=\left(\bigcup \Delta f_{n+11}\right) \cup\left(\bigcup_{m=m_{n}}^{\infty} \delta_{n}^{-m}\right) \cup \delta_{n+1} .
$$

Let us define recurrently the branches $f_{n+1 k}, k=2,3, \ldots$ If $\Delta f_{n+1 k-1} \subset \delta_{n} \backslash \delta_{n+1}$ and $\tilde{G}_{n}: \delta_{n}^{-m} \rightarrow \delta_{n}$, then $f_{n+1 k}=f_{n+1 k-1} \circ \sigma_{n}$. Any branch $f_{n+1 k}$ maps $\tilde{G}_{n}^{-1}\left(\Delta f_{n+1 k-1}\right)$ onto $[0,1]$. For any given $N \in \mathbb{Z}_{+}$we can rewrite (3.6) proceeding as in Sect. 2:

$$
\delta_{n}=\left[\bigcup_{k=1}^{N}\left(\bigcup \Delta f_{n+1 k}\right)\right] \cup\left(\bigcup_{m=m_{n}}^{\infty} \delta_{n+1}^{-m}\right) \cup\left(\bigcup_{m=N \cdot m_{n}}^{\infty} \delta_{n}^{-m}\right) \cup \delta_{n+1}
$$

The preimages $\delta_{n+1}^{-m}$ and $\delta_{n}^{-m}$ in (3.7) have the form $\left(\tilde{G}_{n_{1}} \circ \tilde{G}_{n_{2}}{ }^{\circ} \ldots \circ \tilde{G}_{n_{p}}\right)^{-1} \delta_{n+1}$ (respectively $\delta_{n}$ ) and the branches $f_{n+1 k}$ have the form

$$
f_{n+1 k}=f_{n+11} \circ \tilde{G}_{n_{1}} \circ \tilde{G}_{n_{2}} \circ \ldots \circ \tilde{G}_{n_{p}} .
$$

If $n>I$, there is an infinite number of $\tilde{G}_{n}$, and there is no uniform estimate $\left|D^{2} \tilde{G}_{n}\right|$ $<$ const. However using a generalization of one result of [14] (see Lemma 1 below) we obtain

$$
\lim _{N \rightarrow \infty} \operatorname{mes}\left(\bigcup_{m=N \cdot m_{n}}^{\infty} \delta_{n}^{-m}\right)=0
$$

This implies

$$
\delta_{n}=\left[\bigcup_{k=1}^{\infty}\left(\bigcup \Delta f_{n+1 k}\right)\right] \cup\left[\bigcup_{m=m_{n}}^{\infty}\left(\bigcup \delta_{n+1}^{-m}\right)\right] \cup \delta_{n+1} .
$$

Apart from $\delta_{n}^{-m} \subset \delta_{n} \backslash \delta_{n+1}$ we have $\delta_{n}^{-m} \subset[0,1] \backslash \delta_{n}$ and $\delta_{n}^{-m} \subset \delta_{n+1}$ (domains of $G_{n}$ and $G_{n}^{\prime}$ from (3.1), (3.2), (3.4)). Then (3.9) induces in any such domain $\delta_{n}^{-m}=G_{n}^{-1} \delta_{n}$ the corresponding decomposition

$$
\delta_{n}^{-m}=\left(\bigcup \Delta f_{n+1 k} \circ G_{n}\right) \cup\left(\bigcup \delta_{n+1}^{-m}\right),
$$

where $\delta_{n+1}^{-m}=\mathrm{G}_{n}^{-1} \circ \tilde{\mathrm{G}}_{n_{p}}^{-1} \circ \ldots \circ \tilde{\mathrm{G}}_{n_{1}}^{-1} \delta_{n+1}$.

We shall use $f_{n+1 k}$ to denote $f_{n+1 k-1}{ }^{\circ} G_{n}$ for any $G_{n}$ with domain $\delta_{n}^{-m} \subset[0,1] \backslash \delta_{n} ; f_{n+1}$ to denote $f_{n+1 k}$ for any $k ; F_{n}$ to denote $f_{k} \circ F_{n-1} ; \hat{f}_{n+1}$ to denote $f_{k} \circ \hat{f}_{n}$ for $\hat{f}_{n}$ such that $\Delta \hat{f}_{n} \subset \delta_{n+1}$, and also $\hat{f}_{n+1}$ to denote $f_{n+1}{ }^{\circ} G_{n}$ and 
$f_{n+1} \circ G_{n}^{\prime}$ with $\underset{\sim}{\Delta} G_{n}$ (respectively $\left.\Delta G_{n}^{\prime}\right) \subset \delta_{n+1} ; G_{n+1}$ to denote any composition of the form $\tilde{G}_{n_{1}} \circ \tilde{G}_{n_{2}}{ }^{\circ} \ldots \circ \tilde{G}_{n_{p}} \mid \delta_{n+1}^{-m}$, or $\tilde{G}_{n_{1}} \circ \ldots \circ \tilde{G}_{n_{p}} \circ G_{n} \mid \delta_{n+1}^{-m}$ or $\tilde{G}_{n_{1}} \circ \ldots \circ \tilde{G}_{n_{p}}{ }^{\circ} G_{n}^{\prime} \mid \delta_{n+1}^{-m}$.

With these notations we have:

$$
[0,1]=\left[\bigcup_{k=1}^{n+1}\left(\bigcup \Delta f_{k}\right)\right] \cup\left[\bigcup_{m=1}^{\infty}\left(\bigcup \delta_{n+1}^{-m}\right)\right] \cup \delta_{n+1}
$$

and

$$
\delta_{n+1}=\left(\bigcup \Delta F_{n} \circ g\right) \cup\left(\bigcup \Delta \hat{f}_{n+1}\right) \cup\left[\bigcup_{m=m_{n+1}}^{\infty}\left(\bigcup \delta_{n+1}^{-m}\right)\right] .
$$

(3.11) and (3.12) correspond to (3.1) and (3.2) with $n$ replaced by $n+1$. So we have described Step $n+1$ on the $x$-axis for any $\lambda \in \mathscr{M}_{n}$.

d) According to the induction hypothesis $\mathscr{M}_{n}$ is the union of a countable set of closed intervals with disjoint interiors and some set $\mathscr{F}_{n}$ consisting of limit points of such intervals.

$$
\mathscr{M}_{n}=\left(\bigcup \mathscr{J}_{n}\right) \bigcup \mathscr{F}_{n} .
$$

We assume inductively that $\mathscr{F}_{n} \subset \mathscr{M}$, and define $\mathscr{M}_{n+1} \cap \mathscr{J}_{n}$ for all $\mathscr{J}_{n}$. We fix some positive $\alpha \leqq s / 4$. As $\lambda$ varies over $\mathscr{J}_{n}$, the top of the central branch $F_{n-1} \circ h\left(\lambda, \frac{1}{2}\right)$ varies over some $\Delta f_{k_{0}}$ and $f_{k_{0}} \circ F_{n-1} \circ h\left(\lambda, \frac{1}{2}\right)$ varies over [0,1]. Moreover when $\lambda$ varies in $\mathscr{J}_{n}$ all the maps $F, G, f, \hat{f}$ constructed at previous steps vary continuously. Let $\mathscr{J}_{n}^{\prime}$ be one of these components of $\mathscr{M}_{n}$. In order to construct the set $\mathscr{M}_{n+1} \cap \mathscr{J}_{n}^{\prime}$ we shall point out the admissible positions for the top $f_{k_{0}} \circ F_{n-1} \circ h\left(\lambda, \frac{1}{2}\right)$. Let $\mathscr{J}_{n}^{\prime}$ $=\left[a_{n}, b_{n}\right]$. When constructing $\delta_{n+1}(\lambda)$, we shall choose it varying continuously when $\lambda \in \mathscr{J}_{n}^{\prime}$ and still satisfying (3.5). Then we shall expand $\delta_{n+1}(\lambda)$ almost homothetically and obtain an interval $\hat{\delta}_{n+1}(\lambda)$ varying continuously with $\lambda \in \mathscr{J}_{n}^{\prime}$, composed of whole domains $\Delta f_{k}$ and $\delta_{n+1}^{-m}$ and satisfying for $\lambda \in \mathscr{J}_{n}^{\prime}$ the following

$$
\lambda^{\alpha(n+1)}\left|\delta_{n+1}(\lambda)\right| \leqq\left|\hat{\delta}_{n+2}(\lambda)\right| \leqq \lambda^{\alpha(n+1)}\left(1+0\left(\frac{1}{\lambda^{t(n+1)}}\right)\right)\left|\delta_{n+1}(\lambda)\right|
$$

For any preimage $\delta_{n+1}^{-m}=\mathrm{G}_{n+1}^{-1} \delta_{n+1} C[0,1] \backslash \delta_{n+1}$ the corresponding domain $\hat{\delta}_{n+1}^{-m}$ $=\mathrm{G}_{n+1}^{-1} \hat{\delta}_{n+1}$ turns out to be defined and the lengths of $\delta_{n+1}^{-m}$ and $\hat{\delta}_{n+1}^{-m}$ are still related by (3.13). Then we define

$$
\mathscr{M}_{n+1} \cap \mathscr{J}_{n}=\left\{\lambda: f_{k_{0}} \circ F_{n-1} \circ h\left(\lambda, \frac{1}{2}\right) \in[0,1] \backslash \bigcup_{m} \bigcup \hat{\delta}_{n+1}^{-m}(\lambda)\right\}
$$

The condition $f_{k_{0}} \circ F_{n-1} \circ h\left(\lambda, \frac{1}{2}\right) \in \hat{\delta}_{n+1}^{-m}$ defines an interval in $\mathscr{J}_{n}$. Thus $\mathscr{M}_{n+1} \cap \mathscr{J}_{n}$ is the complement of the union of these intervals. $\mathscr{M}_{n+1} \cap \mathscr{J}_{n}$ consists of intervals $\mathscr{J}_{n k}^{\prime}=\left\{\lambda: f_{k_{0}} \circ F_{n-1} \circ h\left(\lambda, \frac{1}{2}\right) \in \Delta f_{k}(\lambda)\right\}$ and of a limit set $\mathscr{F}_{n+1}\left(\mathscr{J}_{n}\right)$. As $\lambda$ varies over $\mathscr{J}_{n k}^{\prime}$, $f_{k} \circ f_{k_{0}} \circ F_{n-1} \circ h\left(\lambda, \frac{1}{2}\right)$ varies over $[0,1]$.

So we have

$$
\mathscr{M}_{n+1} \cap \mathscr{J}_{n}=\left(\bigcup_{k} \mathscr{J}_{n k}^{\prime}\right) \cup \mathscr{F}_{n+1}\left(\mathscr{J}_{n}\right)
$$

and finally

$$
\mathscr{M}_{n+1}=\left(\bigcup_{\mathscr{I}_{n}}\left(\mathscr{M}_{n+1} \cap \mathscr{J}_{n}\right)\right) \cup \mathscr{F}_{n} .
$$




\section{Estimates for Fluctuation of Derivative}

Let $f: \Delta \rightarrow I$ be a $C^{2}$-diffeomorphism of some closed interval. Then by differentiating $\log |D f(z)|$, we see that

$$
\max _{x, y \in \Delta}\left|\frac{D f(x)}{D f(y)}\right| \leqq \exp \left(\max _{z \in \Delta}\left|\frac{D^{2} f(z)}{D f(z)}\right| \cdot|\Delta|\right) .
$$

We shall use the notation $\mu(f, \Delta)=\max _{x \in \Delta}\left|\frac{D^{2} f(x)}{D f(x)}\right| \cdot|\Delta|$ and when there is no doubt about the domain of $f$, we shall often write $\mu(f)$. Let $f_{1}: \Delta_{1} \stackrel{\text { onto }}{\longrightarrow} I$, $f_{2}: \Delta_{2} \stackrel{\text { onto }}{\longrightarrow} J \supset \Delta_{1}$ be as above, $\Delta_{12}=f_{2}^{-1} \Delta_{1} \subset \Delta_{2}$. Then $f_{1} \circ f_{2}\left(\Delta_{12}\right)=I$. Using the mean value theorem and (4.1) we obtain

$$
\begin{aligned}
\mu\left(f_{1} \circ f_{2}, \Delta_{12}\right)= & \max _{x \in \Delta_{12}}\left|\frac{D^{2}\left(f_{1} \circ f_{2}\right)(x)}{D\left(f_{1} \circ f_{2}\right)(x)}\right|\left|\Delta_{12}\right| \\
= & \max _{x \in \Delta_{12}}\left|\frac{D^{2} f_{1}\left(f_{2}(x)\right) \cdot\left[D f_{2}(x)\right]^{2}+D f_{1}\left(f_{2}(x)\right) \cdot D^{2} f_{2}(x)}{D f_{1}\left(f_{2}(x)\right) \cdot D f_{2}(x)}\right|\left|\Delta_{12}\right| \\
\leqq & \left.\max _{y \in \Delta_{1}}\left|\frac{D^{2} f_{1}(y)}{D f_{1}(y)}\right|\left|\Delta_{1}\right|\right] \cdot\left[\max _{x \in \Delta_{12}}\left|D f_{2}(x)\right| \cdot \frac{\left|\Delta_{12}\right|}{\left|\Delta_{1}\right|}\right] \\
& +\max _{x \in \Delta_{12}}\left|\frac{D^{2} f_{2}(x)}{D f_{2}(x)}\right| \cdot\left|\Delta_{2}\right| \cdot \frac{\left|\Delta_{12}\right|}{\left|\Delta_{2}\right|} \\
\leqq & \mu\left(f_{1}\right) \cdot \max _{x, \theta \in \Delta_{12}}\left|\frac{D f_{2}(x)}{D f_{2}(\theta)}\right|+\mu\left(f_{2}\right) \cdot \frac{\left|\Delta_{12}\right|}{\left|\Delta_{2}\right|} .
\end{aligned}
$$

Since by (4.1)

$$
\max _{x, \theta \in \Delta_{12}}\left|\frac{D f_{2}(x)}{D f_{2}(\theta)}\right| \leqq \exp \left[\mu\left(f_{2}\right) \cdot \frac{\left|\Delta_{12}\right|}{\left|\Delta_{2}\right|}\right]
$$

and

$$
\frac{\left|\Delta_{12}\right|}{\left|\Delta_{2}\right|}=\left|\frac{\mathrm{D} f_{2}\left(\eta_{2}\right)}{D f_{2}\left(\eta_{12}\right)}\right| \frac{\left|\Delta_{1}\right|}{|J|} \leqq\left[\exp \mu\left(f_{2}\right)\right] \frac{\left|\Delta_{1}\right|}{|J|}
$$

we obtain

$$
\max _{x, \theta \in \Delta_{12}}\left|\frac{D f_{2}(x)}{D f_{2}(\theta)}\right| \leqq \exp \left[\mu\left(f_{2}\right)\left\{\exp \mu\left(f_{2}\right)\right\} \frac{\left|\Delta_{1}\right|}{|J|}\right]
$$

Consequently

$$
\begin{aligned}
& \mu\left(f_{1} \circ f_{2} \cdot \Delta_{12}\right) \leqq \mu\left(f_{1}\right) \exp \left[\left\{\mu\left(f_{2}\right) \exp \mu\left(f_{2}\right)\right\} \cdot \frac{\left|\Delta_{1}\right|}{|J|}\right] \\
& +\left\{\mu\left(f_{2}\right) \exp \mu\left(f_{2}\right)\right\} \cdot \frac{\left|\Delta_{1}\right|}{|J|} .
\end{aligned}
$$


Using the notation $v(f, \Delta)=\mu(f, \Delta) \exp \mu(f, \Delta),(4.4)$ is equivalent to

$$
\mu\left(f_{1} \circ f_{2}, \Delta_{12}\right) \leqq \mu\left(f_{1}\right) \exp \left[v\left(f_{2}\right) \cdot \frac{\left|\Delta_{1}\right|}{|J|}\right]+v\left(f_{2}\right) \cdot \frac{\left|\Delta_{1}\right|}{|J|} .
$$

Let $h(x)=a x^{2}$, and let $\Delta$ denote an interval in $\mathbb{R}_{+}$; let $H$ denote the distance from $\Delta$ to 0 , so that $\Delta=(H, H+|\Delta|)$, and suppose $f: \Delta \rightarrow I$ is a $C^{2}$ diffeomorphism. Let $\delta=\left[x_{\min }, x_{\max }\right] \subset \mathbb{R}_{+}$, be one of the two diffeomorphic preimages of $\Delta: d=h^{-1}(\Delta) \cap \mathbb{R}_{+}$. We obtain as above

$$
\mu(f \circ h, \delta) \leqq \mu(f) \cdot \max _{x, y \in \delta}\left|\frac{D h(x)}{D h(y)}\right|+|\Delta| \max _{x, y \in \delta}\left|\frac{D^{2} h(x)}{[D h(y)]^{2}}\right|
$$

and thus

$$
\mu(f \circ h, \delta) \leqq \mu(f) \frac{x_{\max }}{x_{\min }}+\frac{|\Delta|}{2 a x_{\min }^{2}} .
$$

Since $a x_{\max }^{2}=H+|\Delta|$, and $a x_{\min }^{2}=H$, we have

$$
\frac{x_{\max }}{x_{\min }}=\sqrt{1+\frac{|\Delta|}{H}}<1+\frac{|\Delta|}{2 H} .
$$

This implies

$$
\mu(f \circ h, \delta)<\mu(f)\left(1+\frac{|\Delta|}{2 H}\right)+\frac{|\Delta|}{2 H}
$$

or

$$
\mu(f \circ h, \delta)<\mu(f)\left(1+\frac{|\Delta|}{2 a x_{\min }^{2}}\right)+\frac{|\Delta|}{2 a x_{\min }^{2}}
$$

\section{Preliminary Lemma}

We shall use the following several times

Lemma 1. Let $I \cup J=N$ be an interval, $I=\bigcup_{i=1}^{\infty} \Delta \varphi_{i}$, where

1) $\varphi_{i}$ are $C^{2}$-diffeomorphisms from their domains onto $N$;

2) int $\Delta \varphi_{i} \cap \operatorname{int} \Delta \varphi_{j}=\emptyset, i \neq j$;

3) $\left|D \varphi_{i}\right|>\bar{c}_{1}<1$;

4) $\mu\left(\varphi_{i}\right)<\bar{c}_{2}$;

5) $\operatorname{mes} J>0$; $\operatorname{mes} I \cap J=0$.

Then $I=\bigcup_{k=1}^{\infty} \varphi^{-k} J(\bmod 0)$, where $\varphi^{-k} J=\bigcup_{i_{1} \ldots i_{k}} \varphi_{i_{1}}^{-1} \circ \ldots \circ \varphi_{i_{k}}^{-1} J$.

Proof. Since $\varphi_{i}$ is onto, $\Delta \varphi_{i}=\varphi_{i}^{-1} J \cup \varphi_{i}^{-1} I$. Thus

$$
\begin{aligned}
I & =\bigcup_{i} \Delta \varphi_{i}=\bigcup_{i}\left(\varphi_{i}^{-1} J \cup \varphi_{i}^{-1} I\right)=\varphi^{-1} J \cup\left[\bigcup_{i_{1}} \varphi_{i_{1}}^{-1}\left(\bigcup_{i_{2}} \varphi_{i_{2}}^{-1} J \cup \varphi_{i_{2}}^{-1} I\right)\right] \\
& =\varphi^{-1} J \cup \varphi^{-2} J \cup \varphi^{-2} I .
\end{aligned}
$$


In a similar way we obtain for any $N$

$$
I=\bigcup_{k \leqq N} \varphi^{-k} J \cup \varphi^{-N} I .
$$

For any $i_{1}, \ldots, i_{k}$,

$$
\varphi_{i_{1}}^{-1} \circ \ldots \circ \varphi_{i_{k}}^{-1} I=\left[\bigcup_{i} \varphi_{i_{1}}^{-1} \circ \ldots \circ \varphi_{i_{k}}^{-1} \circ \varphi_{i}^{-1} I\right] \cup\left[\bigcup_{i} \varphi_{i_{1}}^{-1} \circ \ldots \circ \varphi_{i_{k}}^{-1} \circ \varphi_{i}^{-1} J\right] .
$$

Suppose there were a constant $\theta>0$ independent of $k$ such that for any $i_{1}, \ldots, i_{k}$

$$
\frac{\operatorname{mes} \varphi_{i_{1}}^{-1} \circ \ldots \circ \varphi_{i_{k}}^{-1} J}{\operatorname{mes} \varphi_{i_{1}}^{-1} \circ \ldots \circ \varphi_{i_{k}}^{-1} I}>\theta \text {. }
$$

Then it would follow from (5.2) that

$$
\operatorname{mes} \varphi^{-(k+1)} I<(1+\theta)^{-1} \operatorname{mes} \varphi^{-k} I,
$$

thus $\lim _{k \rightarrow \infty} \operatorname{mes} \varphi^{-k} I=0$, and in view of (5.1) this would prove Lemma 1. Note that for $k=1,(5.3)$ follows from hypothesis (5).

Consider a $C^{2}$ diffeomorphism $\varphi^{k}=\varphi_{i_{k}}{ }^{\circ}{ }^{\circ} \varphi_{i_{1}}: \varphi_{i_{1}}^{-1} \circ \ldots \circ \varphi_{i_{k}}^{-1} N \rightarrow N$. By the mean value theorem and by (4.1), a proof of (5.3) would follow from a uniform upper bound on the quantities $\mu\left(\varphi^{n}\right)$ independent of $n$. We will show

$$
\mu\left(\varphi^{n}\right)<\left(\sum_{i=0}^{\infty} \frac{\bar{c}_{2} \exp \bar{c}_{2}}{\bar{c}_{1}^{i}}\right) \exp \left(\sum_{i=1}^{\infty} \frac{\bar{x}_{2} \exp \bar{c}_{2}}{\bar{c}_{1}^{i}}\right) .
$$

We prove (5.4) by induction. From (4.5),

$$
\mu\left(\varphi^{n}\right)=\mu\left(\varphi^{n-1} \circ \varphi\right) \leqq \mu\left(\varphi^{n-1}\right) \exp \left[v(\varphi) \frac{\left|\Delta \varphi^{n-1}\right|}{|N|}\right]+v(\varphi) \frac{\left|\Delta \varphi^{n-1}\right|}{|N|} .
$$

According to hypotheses 3 and 4

and

$$
v(\varphi)<\bar{c}_{2} \exp \bar{c}_{2}
$$

$$
\left|\Delta \varphi^{n-1}\right|<|N| / \bar{c}_{1}^{n-1}
$$

Thus

$$
\mu\left(\varphi^{n}\right) \leqq \mu\left(\varphi^{n-1}\right) \exp \left[v(\varphi) / \bar{c}_{1}^{n-1}\right]+v(\varphi) / \bar{c}_{1}^{n-1} .
$$

Suppose for $k \leqq n-1$ that

$$
\mu\left(\varphi^{k}\right) \leqq\left(\sum_{i=0}^{k-1} v(\varphi) / \bar{c}_{1}^{i}\right) \exp \left(\sum_{i=1}^{k-1} v(\varphi) / \bar{c}_{1}^{i}\right) .
$$

(Note that for $k=1$, the second factor above equals 1 and this becomes the obvious inequality $\mu(\varphi)<v(\varphi)$.) Then, using (5.6),

$$
\begin{aligned}
\mu\left(\varphi^{n}\right) & \leqq\left(\sum_{i=0}^{n-2} \frac{v(\varphi)}{\bar{c}_{1}^{i}}\right) \exp \left(\sum_{i=1}^{n-2} \frac{v(\varphi)}{\bar{c}_{1}^{i}}\right) \exp \left(\frac{v(\varphi)}{\bar{c}_{1}^{n-1}}\right)+\frac{v(\varphi)}{\bar{c}_{1}^{n-1}} \\
& \leqq\left(\sum_{i=0}^{n-2} \frac{v(\varphi)}{\bar{c}_{1}^{i}}\right) \exp \left(\sum_{i=1}^{n-1} \frac{v(\varphi)}{\bar{c}_{1}^{i}}\right)+\frac{v(\varphi)}{\bar{c}_{1}^{n-1}} \exp \left(\sum_{i=1}^{n-1} \frac{v(\varphi)}{\bar{c}_{1}^{i}}\right) \\
& \leqq\left(\sum_{i=0}^{n-1} \frac{v(\varphi)}{\bar{c}_{1}^{i}}\right) \exp \left(\sum_{i=1}^{n-1} \frac{v(\varphi)}{\bar{c}_{1}^{i}}\right)
\end{aligned}
$$

and (5.4) is proved. 


\section{Transition from $\boldsymbol{n}$ to $\boldsymbol{n}+\mathbf{1}$, I. Hypotheses of Induction. Estimates of Derivatives}

(3.1) and (3.2) give us the following representation of $[0,1]$ after Step $n$ :

$$
[0,1]=\left[\bigcup_{k=1}^{n}\left(\bigcup \Delta f_{k}\right)\right] \cup\left[\bigcup_{m=1}^{\infty}\left(\bigcup \delta_{n}^{-m}\right)\right] \cup\left(\bigcup \Delta F_{n-1} \circ g\right) \cup\left(\bigcup \Delta \hat{f}_{n}\right)
$$

All domains in (6.1) depend on $\lambda$ which varies in $\mathscr{J}_{n}$, but throughout Sects. 6 and 7. $\lambda$ will be fixed. Any $\delta_{n}^{-m}$ in (6.1) is a preimage of $\delta_{n}$ under some diffeomorphism denoted by $G_{n}$. For given $\delta_{n}^{-m}$ let $p=\max \left\{k: \delta_{n}^{-m} \subset\left[\frac{1}{2}-\frac{1}{\lambda^{s k}}, \frac{1}{2}+\frac{1}{\lambda^{s k}}\right]\right\}$. Then we shall use the notation $G_{n, p}$ for $G_{n}$.

Let $0<s \leqq \frac{1}{13}, 1<\alpha \leqq s / 4$ be constants defined in Sects. 2 and $3, c_{0}=1-s, c_{1}=1$ $-2 s, c_{2}=1-s+\alpha, \gamma=1-3 s, t=\alpha / 10, v=\frac{2(s-\alpha)}{c_{0}}$. Now we formulate the hypotheses of induction.

a) Hypotheses on derivatives:

$$
\begin{aligned}
& \left.\left.a_{1 n}^{1}\right)\left|D f_{k}\right|>2^{k} \cdot \lambda^{c_{1} k}\right\} k=1, \ldots, n \\
& \left.\left.a_{1 n}^{2}\right)\left|D f_{k}\right|>2 \lambda^{c_{0}}\right\} \\
& \left.a_{2 n}\right)\left|D F_{n-1}\right|>2^{n-1} \lambda^{c_{0}(n-1)} \\
& \left.a_{3 n}\right)\left|D \hat{f}_{n}\right|>2^{n} \cdot \lambda^{c_{1} n} \\
& \left.a_{4 n}^{1}\right)\left|D G_{n, p}\right|>\lambda^{s(1-v) p} \\
& \left.a_{4 n}^{2}\right)\left|D G_{n}\right|>2 \lambda^{c_{2} / 2} .
\end{aligned}
$$

b) Hypotheses on $\mu$ :

$$
\begin{aligned}
& \left.b_{1 n}\right) \mu\left(f_{k}\right)<\left(\sum_{i=1}^{k} \frac{1}{2^{i} \cdot \lambda^{\gamma i}}\right) \cdot \prod_{i=1}^{k}\left(1+\frac{1}{2^{i} \cdot \lambda^{\gamma i}}\right) \cdot \exp \left(\sum_{i=1}^{k} \frac{1}{2^{i} \cdot \lambda^{\gamma i}}\right), \quad k=1,2, \ldots, n . \\
& \left.b_{2 n}\right) \mu\left(F_{n-1}\right)<\left(\sum_{i=1}^{n-1} \frac{1}{2^{i} \cdot \lambda^{\gamma i}}\right) \cdot \prod_{i=1}^{n-1}\left(1+\frac{1}{2^{i} \cdot \lambda^{\gamma i}}\right) \cdot \exp \left(\sum_{i=1}^{n-1} \frac{1}{2^{i} \cdot \lambda^{\gamma i}}\right) \\
& \left.b_{3 n}\right) \mu\left(\hat{f}_{n}\right)<\left(\sum_{i=1}^{n} \frac{1}{2^{i} \cdot \lambda^{\gamma i}}\right) \cdot \prod_{i=1}^{n}\left(1+\frac{1}{2^{i} \cdot \lambda^{\gamma i}}\right) \cdot \exp \left(\sum_{i=1}^{n} \frac{1}{2^{i} \cdot \lambda^{\gamma i}}\right) \\
& \left.b_{4 n}\right) \mu\left(G_{n}\right)<\frac{1}{\lambda^{\alpha n}} .
\end{aligned}
$$

We suppose $a_{i n}, b_{i n}$ to be true and we have to prove $a_{i n+1}, b_{i n+1}$.

Remark $V I / 1$. At the beginning of Step $n+1$ we constructed some new preimages $\delta_{n}^{-m}$ with corresponding maps denoted by $G_{n}^{\prime}\left(G_{n}^{\prime}: \delta_{n}^{-m} \rightarrow \delta_{n}\right.$, see Sect. 3). We have to prove that $G_{n}^{\prime}$ also satisfy the conditions $a_{4 n}, b_{4 n}$ which we denote in this case $a_{4 n}^{\prime}$, $b_{4 n}^{\prime}$.

Remark $V I / 2$. Some additional induction hypotheses related to the variation of $\lambda$ will be formulated below. In particular the possibility of choice of intervals $\delta_{n}, \hat{\delta}_{n}$ will be proved, and estimates of sizes of these intervals and their preimages will be given in Sect. 10. Now we shall use (3.5) and (3.13) with $n$ instead of $n+1$ (this is assumed inductively) and with $n+1$ (this will be proved in Sect. 10). One easily checks there is no vicious circle here. 
$\left.a_{1 n+1}\right)$ According to the construction of Sect. $3,\left\{f_{n+1}\right\}=\bigcup_{i=1}^{\infty}\left\{f_{n+1 i}\right\}$ where $f_{n+11}=f_{k} \circ F_{n-1} \circ g_{n}\left(\right.$ with $g_{n}=\lambda \cdot x^{2} \mid\left\{|x|>\frac{1}{\lambda^{s(n+1)}}\right\}$ in local coordinates near $\left.\frac{1}{2}\right)$, or $f_{n+11}=f_{k} \circ \hat{f}_{n}$. In the first case $a_{1 n}, a_{2 n}$ and the form of $g_{n}$ above imply

$$
\left|D f_{n+11}\right| \geqq 2 \lambda^{c_{0}} \cdot 2^{n-1} \cdot \lambda^{c_{0}(n-1)} \cdot 2 \lambda \cdot \frac{1}{\lambda^{s(n+1)}}>2^{n+1} \cdot \lambda^{c_{1}(n+1)} .
$$

In the second case $a_{1 n}, a_{3 n}$ imply

$$
\left|D f_{n+11}\right| \geqq 2 \lambda^{c_{0}} \cdot 2^{n} \cdot \lambda^{c_{1} n}>2^{n+1} \cdot \lambda^{c_{1}(n+1)} .
$$

Thus $a_{1 n+1}^{1}$ is true for $f_{n+11}$. The choice of $s$ implies $2 c_{1}>c_{0}$, hence $a_{1 n}^{1}$ implies $a_{1 n}^{2}$ for $n \geqq 2$. All $f_{n+1 k}, \mathrm{k} \geqq 2$ are compositions of the form $f_{n+1 k}=f_{n+1 k-1} \circ G_{n}$ or $f_{n+1 k}=f_{n+1 k-1}{ }^{\circ} G_{n}^{\prime}$ with $\Delta G_{n}^{\prime} \subset \delta_{n} \backslash \delta_{n+1}$. According to $a_{4 n}^{2},\left|D G_{n}\right|>1$. $\left(a_{4 n}^{2}\right)^{\prime}$ proved below is much stronger than $\left|D G_{n}^{\prime}\right|>1$, and $G_{n}^{\prime}$ under consideration satisfies $\left|D G_{n}^{\prime}\right|$ $>2^{n} \cdot \lambda^{c_{1} n}$. Indeed, $G_{n}^{\prime}=G_{n} \circ F_{n-1} \circ g_{n}$ or $G_{n}^{\prime}=G_{n} \circ \hat{\mathrm{f}}_{n}$. In both cases $a_{2 n}$ and $a_{3 n}$ imply as above $\left|D G_{n}^{\prime}\right|>2^{n} \cdot \lambda^{c_{1} n}$.

$\left.a_{2 n+1}\right) F_{n}=f_{k} \circ F_{n-1}$. Hence $a_{1 n}$ and $a_{2 n}$ imply $a_{2 n+1}$.

$\left.a_{4 n}^{2 \prime}\right)$ We consider $G_{n}: \delta_{n}^{-m} \rightarrow \delta_{n}, G_{n}^{\prime}=G_{n} \circ F_{n-1} \circ g$ or $G_{n}^{\prime}=G_{n} \circ \hat{f}_{n}$ and their domains $\delta_{n}^{-N}=\left(F_{n-1} \circ g\right)^{-1}\left(\delta_{n}^{-m}\right)$ or $\delta_{n}^{-M}=\hat{f}_{n}^{-1}\left(\delta_{n}^{-m}\right)$. The most complicated is the case of central branch $F_{n-1} \circ$. We omit indices and use $\delta$ to denote $\delta_{n}^{-m}$ (if $m=0$, $\left.\delta=\delta_{n}\right), G$ to denote $G_{n}$ (if $m=0, G=\mathrm{id}$ ), $\ell$ to denote $\left(F_{n-1} \circ h\right)^{-1} \delta$. We estimate $\left|D\left(G \circ F_{n-1} \circ h\right)\right|$. Let $H=\operatorname{dist}\left(\delta, F_{n-1} \circ h\left(\frac{1}{2}\right)\right)$. The induction construction of Step $n$ implies that the top $F_{n-1} \circ h\left(\frac{1}{2}\right)$ lies outside an interval $\hat{\delta}$ corresponding to $\delta$. Thus (see (3.13) with $n$ instead of $n+1$ )

$$
H \geqq\left(\lambda^{\alpha n}-1\right) \cdot|\delta| / 2 .
$$

Let $H_{1}=\operatorname{dist}\left(F_{n-1}^{-1} \delta, h\left(\frac{1}{2}\right)\right)$. It follows from (4.1) and $b_{2 n}$ that

$$
H_{1}>\left(\lambda^{\alpha n}-1\right) \cdot\left|F_{n-1}^{-1} \delta\right| \cdot 2^{-1} \cdot \exp \left(-\frac{1+\varepsilon_{6.2}}{2 \lambda^{\gamma}}\right), \quad \text { where } \varepsilon_{6.2}=O\left(\lambda^{-\gamma}\right)
$$

Remark $V I / 3$. Several constants $0 \leqq \varepsilon_{i, k}<\lambda^{-t}$ are indexed according to the numbers of inequalities in which they occur.

Let $\ell=\left[x_{\min }, x_{\max }\right]$. We have, using the local coordinate,

$$
h\left(x_{\min }\right)=\lambda \cdot x_{\min }^{2}=H_{1}, \quad x_{\min } \sqrt{H_{1} \lambda^{-1}}, \quad|D h|_{\ell}|\geqq 2 \lambda| x_{\min } \mid=2 \sqrt{\lambda \cdot H_{1}} .
$$

In consequence of $|\delta|=\left|F_{n-1}^{-1} \delta\right| \cdot\left|D F_{n-1}(\theta)\right|$, for some $\theta \in F_{n-1}^{-1} \delta$, we obtain

$$
|D h|_{\ell} \mid \geqq \sqrt{\frac{2 \cdot \lambda^{\alpha n+1}|\delta|}{\left|D F_{n-1}(\theta)\right|}\left(1-\varepsilon_{6.3}\right)}
$$

Since $\left|D\left(F_{n-1} \circ h\right)\right|=\left|D F_{n-1}\right| \cdot|D h|$, we have, using (4.1) and $b_{2 n}$, for any $x \in \Delta F_{n-1}$

$$
\left|D\left(F_{n-1} \circ h\right)\right|_{\ell} \geqq \sqrt{2 \cdot \lambda^{\alpha n+1} \cdot\left|D F_{n-1}(x)\right| \cdot|\delta|}\left(1-\varepsilon_{6.4}\right)
$$


If $\delta=\delta_{n}$, then (6.4), $\left|\delta_{n}\right|>2 \cdot \lambda^{-s n}$ and $a_{2 n}$ imply

$$
\left|D\left(F_{n-1} \circ h\right)\right|_{\ell} \mid \geqq\left(\sqrt{2} \cdot \lambda^{\frac{1}{2}\left(c_{1}+\alpha\right)}\right)^{n} \cdot \sqrt{2 \lambda^{s}} \cdot\left(1-\varepsilon_{6.5}\right) .
$$

If $\delta=\delta_{n}^{-m}=G^{-1} \delta_{n}$ we obtain, using $a_{4 n}$

$$
\left|D\left(G \circ F_{n-1} \circ h\right)\right|_{\ell} \mid \geqq\left(\sqrt{2} \cdot \lambda^{\frac{1}{2}\left(c_{1}+\alpha\right)}\right)^{n} \sqrt{\lambda^{s+c_{2} / 2}} \cdot 2\left(1-\varepsilon_{6.6}\right) .
$$

(6.5) and (6.6) imply $\left(a_{4 n}^{2}\right)^{\prime}$ for $G_{n}^{\prime}=G_{n} \circ F_{n-1} \circ n$. In the case $G_{n}^{\prime}=G_{n} \circ F_{n-1} \circ g$ we have in (6.2) $H_{1}>\frac{1}{2} \cdot\left|\Delta F_{n-1}\right| \exp \left(-\frac{1+\varepsilon_{6.2}}{2 \lambda^{\gamma}}\right)$, which leads to better estimates. In the case $G_{n}^{\prime}=G_{n} \circ \hat{f}_{n}\left(a_{4 n}^{2}\right)^{\prime}$ is obvious because of $a_{3 n}$ and $a_{4 n}^{2}$.

$\left.a_{4 n+1}^{2}\right)$ Follows from $a_{4 n}^{2},\left(a_{4 n}^{2}\right)^{\prime}$ and the definition:

$G_{n+1}=G_{n_{1}} \circ \ldots \circ G_{n_{p}}$.

$\left.a_{3 n+1}\right)$ If $\hat{f}_{n+1}=f_{n+1} \circ G_{n_{1}} \circ \ldots \circ G_{n_{p}}, a_{3 n+1}$ follows from $a_{1 n+1}, a_{4 n}^{2}$ and $\left(a_{4 n}^{2}\right)^{\prime}$. If $\hat{f}_{n+1}=f_{k} \circ \hat{f}_{n}, a_{3 n+1}$ follows from $a_{1 n}$ and $a_{3 n}$.

Remark VI/4. The inequalities (6.5), (6.6) show that the derivatives of $G_{n}^{\prime}$ grow exponentially with $n$, but this is not sufficient to prove $\left(a_{4 n}^{1}\right)^{\prime}$. Indeed, let $n_{1}$ be so that $F_{n_{1}-1} \circ h\left(\lambda, \frac{1}{2}\right)$ may lie in the domain $\delta_{1}$. As $\delta_{1}$ contains $\Delta f_{2}$ of arbitrary small diameter, the interval $\Delta\left(f_{2} \circ F_{n_{1}-1} \circ h\right)=\Delta\left(F_{n_{1}} \circ h\right)$ may also be arbitrarily small and the corresponding $\delta_{n_{1}}^{-M}=\left(F_{n_{1}} \circ h\right)^{-1} \delta_{n_{1}}$ is contained in $\delta_{N}$ with arbitrarily large $N$. However $\left|D F_{n_{1}}\right|$ turns out to be very large in this case, which implies $\left(a_{4 n}^{1}\right)^{\prime}$.

$\left.a_{4 n}^{1}\right)$ We use the notation introduced in the proof of $\left(a_{4 n}^{2}\right)^{\prime}$. According to the definition, the domain $\ell=\left(F_{n-1} \circ h\right)^{-1} \delta$ of $G_{n, p}^{\prime}$ is so that $\ell \subset\left(\frac{1}{2}-\lambda^{-s p}, \frac{1}{2}+\lambda^{-s p}\right)$, but $\ell \not \subset\left(\frac{1}{2}-\lambda^{-s(p+1)}, \frac{1}{2}+\lambda^{-s(p+1)}\right)$. Let $\ell \subset\left(\frac{1}{2}, \frac{1}{2}+\lambda^{-s p}\right)$. Then $H_{1}=\lambda x_{\min }^{2}<\lambda^{1-2 s p}$. It follows from (6.2)

$$
\frac{\lambda}{\lambda^{2 s p}}>\frac{\lambda^{\alpha n}}{2} \cdot \frac{|\delta|\left(1-\varepsilon_{6.7}\right)}{\left|D F_{n-1}\left(\theta_{1}\right)\right|} .
$$

(6.7) together with $b_{2 n}$ imply for any $x \in \Delta F_{n-1}$

$$
\left|D F_{n-1}(x)\right|>\frac{\lambda^{\alpha n-1} \cdot \lambda^{2 s p}}{2} \cdot|\delta|\left(1-\varepsilon_{6.8}\right)
$$

Thus we can rewrite (6.4) as

$$
\left|D\left(F_{n-1} \circ h\right)\right|_{\ell}\left|\geqq \lambda^{\alpha n} \cdot \lambda^{s p} \cdot\right| \delta \mid \cdot\left(1-\varepsilon_{6.9}\right) .
$$

From $|\delta|=\frac{\left|\delta_{n}\right|}{\left|D G_{n}\left(\theta_{2}\right)\right|}$, and $\left|\delta_{n}\right|>2 \cdot \lambda^{-s n}$ we obtain using $b_{4 n}$

$$
\left|D G_{n, p}\right|=\left|D\left(G_{n} \circ F_{n-1} \circ h\right)\right|_{\ell} \mid \geqq 2 \cdot \lambda^{\alpha n} \cdot \lambda^{s(p-n)} \cdot\left(1-\varepsilon_{6.10}\right) \text {. }
$$

Let us compare $p$ and $n$. Let $\mathscr{D}_{n-1}=\left(\frac{1}{2}-u_{n-1}^{1}, \frac{1}{2}+u_{n-1}^{2}\right)$ be the domain of $F_{n-1} \circ h$, and $p_{1}=\max \left\{q: u_{n-2}^{2}<\lambda^{-s q}\right\}$. Then $p \geqq p_{1}$. We have in the local coordinate system, using $a_{2 n}$,

$$
2^{n-1} \cdot \lambda^{c_{0}(n-1)+1} \cdot\left(u_{n-1}^{2}\right)^{2}<F_{n-1} \circ h\left(\frac{1}{2}\right) \leqq 1 .
$$


Thus

$$
\lambda^{-s\left(p_{1}+1\right)}<u_{n-1}^{2}<\left[(\sqrt{2})^{n-1} \lambda^{\left(c_{0} n+s\right) 1 / 2}\right]^{-1} .
$$

(6.11) implies $n<2 s\left(p_{1}+\frac{1}{2}\right) / c_{0}$, which gives for $v$ a somewhat worse estimate than $2(s-\alpha) / c_{0}$. We prefer to improve it instead of taking a different $v$. It suffices to make $F_{n-1} \circ h\left(\lambda, \frac{1}{2}\right)$ lie outside $\left(\frac{1}{2}-\lambda^{-s / 2}, \frac{1}{2}+\lambda^{-s / 2}\right)$ for the first two steps. This gives an extra factor $\lambda^{-s / 2}$ on the right side of (6.11). Hence

$$
n<\frac{2 s}{c_{0}} p_{1}
$$

Remark $V I / 5$. For a given $n_{0}$ we may introduce the additional condition

$$
F_{n-1} \circ h\left(\lambda, \frac{1}{2}\right) \in[0,1] \backslash\left[\frac{1}{2}-\lambda^{-s / 2}, \frac{1}{2}+\lambda^{-s / 2}\right] \quad n \leqq n_{0}
$$

(above $n_{0}=2$ ). This simplifies the estimates, but as follows from Sect. 11, it gives an extra factor of $\left(1-2 N_{0}^{-s / 2}\right)^{n_{0}}$ in the estimate of $\mathscr{M}$. However, this factor can be made arbitrarily close to 1 by taking $N_{0}$ sufficiently large.

As $p \geqq p_{1},(6.10)$ and (6.12) imply

$$
\left|D G_{n, p}^{\prime}\right|>\lambda^{p s\left(1-\frac{2(s-\alpha)}{c_{0}}\right)}=\lambda^{p s(1-v)}
$$

which finishes the proof of $\left(a_{4 n}^{1}\right)^{\prime}$ for $\ell=\left(F_{n-1} \circ h\right)^{-1} \delta$.

If $G_{n, p}^{\prime}=G_{n} \circ F_{n-1} \circ g$, the estimate of $H_{1}$ (see the proof of $\left.\left(a_{4 n}^{2}\right)^{\prime}\right)$ implies that (6.10) turns out into $\left|D G_{n, p}^{\prime}\right|>\lambda^{s p}\left(1-\varepsilon_{6.10}\right)$. Finally when $G_{n, p}^{\prime}=G_{n} \circ \hat{f}_{n}$, notice that any $\hat{f}_{n}$ is a composition of the form $\varphi \circ G_{k}, k \leqq n-1$, where $G_{k}$ satisfies $a_{4 k}^{1}$, and $|D \varphi|$ $>1$.

$$
\left.a_{4 n+1}^{1}\right) \text { Follows from } a_{4 n}^{1}, a_{4 n}^{2},\left(a_{4 n}^{1}\right)^{\prime},\left(a_{4 n}^{2}\right)^{\prime} \text { and the definition of } G_{n+1} \text {. }
$$

\section{Transition from $n$ to $n+1$, II. Estimates of $\mu$}

$\left.b_{4 n+1}\right)$ Let $G_{n}^{\prime}=G_{n} \circ F_{n-1} \circ h: I \rightarrow \delta_{n}$, where

$$
G_{n}: \Delta G_{n}=\delta_{n}^{-m} \rightarrow \delta_{n}, \text { and } \ell=\left(F_{n-1} \circ\right)^{-1} \delta_{n}^{-m} .
$$

We estimate $\mu\left(G_{n}^{\prime}, \ell\right)$ first.

According to (4.5)

$$
\mu\left(G_{n} \circ F_{n-1}, F_{n-1}^{-1} \Delta G_{n}\right) \leqq \mu\left(G_{n}\right) \exp \left(v\left(F_{n-1}\right) \cdot\left|\Delta G_{n}\right|\right)+v\left(F_{n-1}\right) \cdot\left|\Delta G_{n}\right| .
$$

We have

$$
\left|\delta_{n}\right|=\left|\Delta G_{n}^{\prime}\right| \cdot\left|D G_{n}\left(\theta_{0}\right)\right|, \quad\left|\delta_{n}\right|<2 \cdot \lambda^{-s n}\left(1+O\left(\lambda^{-t n}\right)\right), \quad\left|D G_{n}\right|>2 \lambda^{c_{2} / 2} .
$$

In consequence of $b_{2 n}, v\left(F_{n-1}\right)=1+O\left(\lambda^{-\gamma}\right)$. Thus we obtain, using $a_{2 n}, b_{4 n}$ :

$$
\begin{aligned}
\mu\left(G_{n} \circ F_{n-1}, F_{n-1}^{-1} \Delta G_{n}\right)< & \lambda^{-\alpha n} \cdot \exp \left[\frac{2\left(1+O\left(\lambda^{-\gamma}\right)\right)}{\left|D G_{n}\left(\theta_{0}\right)\right| \lambda^{c_{0}(n-1)+s n} \cdot 2^{n-1}}\right] \\
& +\frac{2\left(1+O\left(\lambda^{-\gamma}\right)\right)}{\left|D G_{n}\left(\theta_{0}\right)\right| \lambda^{c_{0}(n-1)+s n} \cdot 2^{n-1}}=\frac{1+\varepsilon_{7.1}}{\lambda^{\alpha n}} .
\end{aligned}
$$


Proceeding along the line of the proof of (6.2), and using (7.1) and (4.6) with $\Delta=F_{n-1}^{-1} \Delta G_{n}, H>\frac{1}{2}\left(1-O\left(\lambda^{-\gamma}\right)\right) \cdot \lambda^{\alpha n} \cdot|\Delta|$ we obtain

$$
\mu\left(G_{n} \circ F_{n-1} \circ h, \ell\right)<\frac{1+\varepsilon_{7.1}}{\lambda^{\alpha n}}\left(1+\frac{1+O\left(\lambda^{-\gamma}\right)}{\lambda^{\alpha n}}\right)+\frac{1+O\left(\lambda^{-\gamma}\right)}{\lambda^{\alpha n}}<\frac{2\left(1+\varepsilon_{7.2}\right)}{\lambda^{\alpha n}} .
$$

The proof for $G_{n}^{\prime}=G_{n} \circ F_{n-1} \circ g$ and $G_{n}^{\prime}=G_{n} \circ \hat{f}_{n}$ is similar and gives a better estimate

$$
\mu\left(G_{n}^{\prime}\right)<\left(1+\varepsilon_{7.3}\right) \lambda^{-\alpha n} .
$$

Then we consider $G_{n+1}=\tilde{G}_{n_{1}} \circ \ldots \circ \tilde{G}_{n_{p}}: \delta_{n}^{-M} \rightarrow \delta_{n}, \delta_{n}^{-M} C \delta_{n} \backslash \delta_{n+1}$. When estimating $\mu\left(G_{n+1}, \delta_{n}^{-M}\right)$ we use the proof of Lemma 1 with $\varphi_{i}=\widetilde{G}_{n_{i}}, \bar{c}_{2}=\left(1+\varepsilon_{7.3}\right) \lambda^{-\alpha n}$, according to (7.3) and $b_{4 n}$, and $\bar{c}_{1}=\max \left(\lambda^{c_{2} / 2}, \lambda^{s[n(1-v)-1]}\right)$, according to $a_{4 n}$. Then (5.4) gives

$$
\mu\left(G_{n+1}, \delta_{n}^{-M}\right)<\left(1+\varepsilon_{7.4}\right) \lambda^{-\alpha n} .
$$

The estimates (3.5) of $\left|\delta_{n}\right|$ and $\left|\delta_{n+1}\right|$ imply

$$
\frac{\left|\delta_{n+1}\right|}{\left|\delta_{n}\right|}<\frac{1+\varepsilon_{7.5}}{\lambda^{s}}
$$

Considering $\delta_{n+1}^{-M}=G_{n+1}^{-1} \delta_{n+1}$ and applying (7.4), (7.5) we obtain

$$
\begin{aligned}
\mu\left(G_{n+1}, \delta_{n+1}^{-M}\right) \leqq & \mu\left(G_{n+1}, \delta_{n}^{-M}\right) \cdot \frac{\left|\delta_{n+1}^{-M}\right|}{\left|\delta_{n}^{-M}\right|} \leqq \mu\left(G_{n+1}, \delta_{n}^{-M}\right) \cdot \frac{\left|\delta_{n+1}\right|}{\left|\delta_{n}\right|} \\
& \cdot \exp \mu\left(G_{n+1}, \delta_{n}^{-M}\right)<\frac{\left(1+\varepsilon_{7.4}\right)\left(1+\varepsilon_{7.5}\right)\left(1+\varepsilon_{7.6}\right)}{\lambda^{\alpha n+s}}<\frac{1}{\lambda^{\alpha(n+1)}}
\end{aligned}
$$

which proves $b_{4 n+1}$ for $\delta_{n+1}^{-M} \subset \delta_{n} \backslash \delta_{n+1}$.

Any $G_{n+1}: \delta_{n+1}^{-N} \rightarrow \delta_{n+1}$ for $\delta_{n+1}^{-N} \subset[0,1] \backslash\left(\delta_{n} \backslash \delta_{n+1}\right)$ is either a restriction of $G_{n}: \delta_{n}^{-N} \rightarrow \delta_{n}$ on $\delta_{n+1}^{-N} \subset \delta_{n}^{-N}$, or a composition of the form $\tilde{G}_{n+1}{ }^{\circ} G_{n}$ or $\tilde{G}_{n+1}{ }^{\circ} G_{n}^{\prime}$, where $\mu\left(\tilde{G}_{n+1}\right)$ satisfies (7.6), $\mu\left(G_{n}\right)$ satisfies $b_{4 n}$ and $\mu\left(G_{n}^{\prime}\right)$ satisfies (7.2). The case of restriction is treated along the lines of (7.5), (7.6). In the other cases, (4.5) together with $a_{4 n}^{2}$ imply

$$
\mu\left(G_{n+1}, \delta_{n+1}^{-N}\right) \leqq \prod_{i=4}^{6}\left(1+\varepsilon_{7.1}\right) \cdot \frac{1}{\lambda^{\alpha n+s}} \exp \left(\frac{\exp \left(3 \cdot \lambda^{-\alpha n}\right)}{2 \lambda^{\alpha n+c_{2} / 2}}\right)+\frac{\exp \left(3 \cdot \lambda^{-\alpha n}\right)}{2 \lambda^{\alpha n+c_{2} / 2}}
$$

which proves $b_{4 n+1}$.

$$
\left.b_{1 n+1}\right)\left\{f_{n+1}\right\}=\bigcup_{k=1}^{\infty}\left\{f_{n+1 k}\right\} \text {, where } f_{n+11}=f_{k} \circ F_{n-1} \circ g_{n}, k \in[1, n] \text {, or } f_{n+11}
$$
$=f_{k} \circ \hat{f}_{n}$, and $f_{n+1 k}$ are obtained from $f_{n+11}$ using consecutive compositions with different sorts of $\tilde{G}_{n}$ and $G_{n}$. Let us begin with $f_{n+11}=f_{k} \circ F_{n-1} \circ g_{n}$. (4.5) implies:

$$
\mu\left(f_{k} \circ F_{n-1}\right) \leqq \mu\left(f_{k}\right) \exp \left(v\left(F_{n-1}\right) \cdot\left|\Delta f_{k}\right|\right)+v\left(F_{n-1}\right) \cdot\left|\Delta f_{k}\right| \text {. }
$$

We have $v\left(F_{n-1}\right)=\frac{1+\varepsilon_{7.8}}{2 \lambda^{\gamma}}$ (in consequence of $\left.b_{2 n}\right),\left|\Delta f_{k}\right|<\frac{1}{2^{k} \lambda^{c_{1} k}}$ (in consequence of $\left.a_{1 n}\right)$, thus

$$
\mu\left(f_{k}^{\circ} F_{n-1}\right) \leqq \mu\left(f_{k}\right) \exp \left(\frac{1+\varepsilon_{7.8}}{2^{k+1} \cdot \lambda^{c_{1} k+\gamma}}\right)+\frac{1+\varepsilon_{7.8}}{2^{k+1} \cdot \lambda^{c_{1} k+\gamma}}
$$


Let $\Delta$ be the domain of $f_{k} \circ F_{n-1}$. Then (3.5) and (4.7) used with $a=\lambda$, imply

$$
\mu\left(f_{k} \circ F_{n-1} \circ g_{n}\right) \leqq \mu\left(f_{k} \circ F_{n-1}\right)\left(1+|\Delta| \cdot \lambda^{2 s(n+1)-1}\right)+|\Delta| \cdot \lambda^{2 s(n+1)-1} .
$$

We have $|\Delta|<2^{-n} \cdot \lambda^{-c_{0} n}$, because of $a_{1 n}^{2}$ and $a_{2 n}$, and thus

$$
\mu\left(f_{k} \circ F_{n-1} \circ g_{n}\right) \leqq \mu\left(f_{k} \circ F_{n-1}\right)\left(1+\frac{1}{\lambda^{s} \cdot 2^{n} \cdot \lambda^{\left(c_{0}-2 s\right)(n+1)}}\right)+\frac{1}{\lambda^{s} \cdot 2^{n} \cdot \lambda^{\left(c_{0}-2 s\right)(n+1)}} .
$$

In a similar way one verifies using $a_{3 n}$ and $b_{3 n}$ that $\mu\left(f_{n+11}=f_{k} \circ \hat{f}_{n}\right)$ also satisfies (7.8).

Using $b_{1 n},(7.8)$ and (7.9), we obtain

$$
\begin{aligned}
\mu\left(f_{n+11}\right) \leqq & {\left[\left(\sum_{i=1}^{k} \frac{1}{2^{i} \lambda^{\gamma i}}\right) \cdot \prod_{i=1}^{k}\left(1+\frac{1}{2^{i} \lambda^{\gamma i}}\right) \cdot\left(\exp \sum_{i=1}^{k} \frac{1}{2^{i} \lambda^{\gamma i}}\right)\right.} \\
& \left.\cdot \exp \left(\frac{1+\varepsilon_{7.8}}{2^{k+1} \lambda^{c_{1} k+\gamma}}\right)+\frac{1+\varepsilon_{7.8}}{2^{k+1} \lambda^{c_{1} k+\gamma}}\right] \\
& \cdot\left(1+\frac{1}{\lambda^{s} \cdot 2^{n} \cdot \lambda^{\left(c_{0}-2 s\right)(n+1)}}\right)+\frac{1}{\lambda^{s} \cdot 2^{n} \cdot \lambda^{\left(c_{0}-2 s\right)(n+1)}} .
\end{aligned}
$$

Since $c_{1}-s=c_{0}-2 s=\gamma$, we have

$$
\left(1+\varepsilon_{7.8}\right) \cdot 2^{-(k+1)} \cdot \lambda^{-c_{1} k-\gamma}=\left(1+\varepsilon_{7.8}\right) \cdot 2^{-(k+1)} \cdot \lambda^{-\gamma(k+1)} \cdot \lambda^{-s k} \ll 2^{-(k+1)} \cdot \lambda^{-\gamma(k+1)}
$$

and

$$
\lambda^{-s} \cdot 2^{-n} \cdot \lambda^{-\left(c_{0}-2 s\right)(n+1)}=\left(2 \lambda^{-s}\right) \cdot 2^{-(n+1)} \cdot \lambda^{-\gamma(n+1)}<2^{-(n+1)} \cdot \lambda^{-\gamma(n+1)} .
$$

Therefore

$$
\begin{aligned}
& \mu\left(f_{n+11}\right)<\left(\sum_{i=1}^{k} \frac{1}{2^{i} \lambda^{\gamma i}}\right) \cdot\left(\exp \sum_{i=1}^{k+1} \frac{1}{2^{i} \lambda^{\gamma i}}\right) \cdot\left(\prod_{i=1}^{k+1}\left(1+\frac{1}{2^{i} \lambda^{\gamma i}}\right)\right) \\
& +\frac{1}{2^{k+1} \cdot \lambda^{\gamma(k+1)}}\left[\frac{\left(1+\varepsilon_{7.8}\right)\left[1+\left(2 \lambda^{-s}\right) \cdot 2^{-(n+1)} \cdot \lambda^{-\gamma(n+1)}\right]}{\lambda^{s k}}+\frac{2}{\lambda^{s} \cdot 2^{n-k} \cdot \lambda^{\gamma(n-k)}}\right] .
\end{aligned}
$$

Since $k \leqq n$, the factor in square brackets is less than 1 , which implies $b_{1 n+1}$ for $f_{n+11}$.

If $f_{n+1 k}=f_{n+11} \circ \tilde{G}_{n+1}=f_{n+11} \circ \tilde{G}_{n_{1}}{ }^{\prime} \ldots \circ \tilde{G}_{n_{p}}$ we have using (4.5), (3.5), (7.4) and $a_{1 n+1}^{1}$

$$
\mu\left(f_{n+1 k}\right) \leqq \mu\left(f_{n+11}\right) \exp \left(\frac{1+\varepsilon_{7.12}}{2^{n+1} \cdot \lambda^{s+\alpha n+\gamma(n+1)}}\right)+\frac{1+\varepsilon_{7.12}}{2^{n+1} \cdot \lambda^{s+\alpha n+\gamma(n+1)}} .
$$

Substituting (7.10) in (7.12) we obtain $b_{1 n+1}$ as above. The same reasoning proves $b_{1 n+1}$ for $f_{n+1 k}=f_{n+11} \circ \tilde{G}_{n+1} \circ G_{n}$.

$b_{2 n+1}$ ) The proof is similar to the above proof of $b_{1 n+1}$.

$\left.b_{3 n+1}\right)$ For $\hat{f}_{n+1}=f_{k} \circ \hat{f}_{n}$ with $\Delta \hat{f}_{n} \subset \delta_{n+1}$ and for $\hat{f}_{n+1}=f_{n+1} \circ G_{n}$ the proof is similar. For $\hat{f}_{n+1}=f_{n+1}{ }^{\circ} G_{n}^{\prime}(7.2)$ is applied. 


\section{Measure of Holes After Step $n+1$}

For any $\lambda \in \mathscr{M}_{n}$ we estimate the measure of the union $\delta_{n}(\lambda) \cup \bigcup_{m=1}^{\infty}\left(\bigcup \delta_{n}^{-m}(\lambda)\right)$, where $\delta_{n}^{-m}(\lambda) \subset[0,1] \backslash \delta_{n}(\lambda)$.

Lemma 2. There exists an $\varepsilon<\lambda^{-t}$ so that for any $k \in \mathbb{Z}_{+} \backslash\{0\}$

$$
\operatorname{mes}\left[\delta_{k} \cup \bigcup_{m=1}^{\infty}\left(\bigcup \delta_{k}^{-m}\right)\right]<\frac{(1+\varepsilon)^{k}}{\lambda^{s k}} \text {. }
$$

Proof. We proceed by induction and assume that after Step $n$ :

i) The estimate of Lemma 2 holds for $k=n$;

ii) To any hole $\delta_{n}^{-m}$ there corresponds a unique hole $\delta_{n-1}^{-m} \supset \delta_{n}^{-m}$ and a set $K_{n, m}$ $=K_{n, m}\left(\delta_{n}^{-m}\right) \subset \delta_{n-1}^{-m}$, such that $K_{n, m} \subset \mathscr{X}_{n}$ and for some $\varepsilon_{8.1}=O\left(\lambda^{-t}\right)$

$$
\frac{\operatorname{mes} \delta_{n}^{-m}}{\operatorname{mes} K_{n, m}}<\frac{1+\varepsilon_{8.1}}{\lambda^{s}} \text {. }
$$

Remark VIII/1. The proof of Lemma 4 in Sect. 10 implies ii above. However we prove ii here in order to separate the proof of Lemma 2.

Remark VIII/2. We shall use here that the intervals $\delta_{n}, \delta_{n+1}$ constructed in Sect. 10 are chosen so as to have $\delta_{n-1}^{-m} \subset \delta_{n} \backslash \delta_{n+1}$ for the holes $\delta_{n-1}^{-m}$ corresponding to holes $\delta_{n}^{-m} \subset \delta_{n} \backslash \delta_{n+1}$.

We began Step $n+1$ by taking compositions $f_{k} \circ\left(F_{n-1} \circ g\right)$ or $f_{k} \circ \hat{f}_{n}$ and creating new holes of the form $\left(F_{n-1} \circ g\right)^{-1} \delta_{n}^{-m}, \hat{f}_{n}^{-1} \delta_{n}^{-m}$. Let $\delta_{n n+1}=\delta_{n} \backslash \delta_{n+1}$. There are holes $\delta_{n}^{-m}$ of two kinds inside $\delta_{n n+1}$ : the old ones $\delta_{n}^{-m} \subset \delta_{n-1}^{-m}$, and the new ones $\tilde{\delta}_{n}^{-M}=\left(F_{n-1} \circ g_{n}\right)^{-1} \delta_{n}^{-m}$, or $\tilde{\delta}_{n}^{-M}=\hat{f}_{n}^{-1} \delta_{n}^{-m}$ for $\Delta \hat{f}_{n} \subset \delta_{n n+1}, m=0,1, \ldots$. Let

$$
p_{n n+1}=\operatorname{mes}\left[\left(\bigcup \delta_{n}^{-m}\right) \bigcap \delta_{n n+1}\right], \quad \tilde{p}_{n n+1}=\operatorname{mes}\left[\left(\bigcup \tilde{\delta}_{n}^{-m}\right) \bigcap \delta_{n n+1}\right] \text {. }
$$

Then (8.1) implies

$$
p_{n+1}<\left|\delta_{n n+1}\right| \cdot\left(1+\varepsilon_{8.1}\right) \cdot \lambda^{-s} .
$$

One obtains similarly to (7.9) $\mu\left(F_{n-1} \circ g_{n}\right)<1+\lambda^{-\gamma}$. Then i) implies

$$
\begin{aligned}
\tilde{p}_{n n+1} & <\left|\delta_{n n+1}\right| \cdot\left(\exp \mu\left(F_{n-1} \circ g_{n}\right)\right) \cdot\left[\left(1+\varepsilon_{8.1}\right) \cdot \lambda^{-s}\right]^{n} \\
& <\left|\delta_{n n+1}\right| \cdot\left(1+\lambda^{-\gamma}\right) \cdot\left[\left(1+\varepsilon_{8.1}\right) \cdot \lambda^{-s}\right]^{n} .
\end{aligned}
$$

The construction of Sect. 3 implies the one-to-one correspondence between $\delta_{n+1}^{-m}$ and corresponding $\delta_{n}^{-m}\left(\delta_{n+1}^{-m} \subset \delta_{n}^{-m} \subset \delta_{n n+1}\right)$. We have, according to the construction, $\quad \operatorname{mes}\left(\bigcup \Delta f_{n+11}\right) \geqq\left(\operatorname{mes} \delta_{n n+1}\right)-p_{n n+1}-\tilde{p}_{n n+1}$. Now, we let $K_{n+1}$ $=K_{n+1,0}=\bigcup \Delta f_{n+11}$ correspond to $\delta_{n+1}$. In consequence of (8.2) and (8.3) we have

$$
\begin{aligned}
\frac{\operatorname{mes} \delta_{n+1}}{\operatorname{mes} K_{n+1}}< & \frac{2\left(1+o\left(\lambda^{-t(n+1)}\right)\right.}{\lambda^{s(n+1)}} \\
& :\left[\frac{2}{\lambda^{s n}}\left(1-\frac{1+o\left(\lambda^{-t(n+1)}\right)}{\lambda^{s}}\right) \cdot\left(1-\frac{1+\varepsilon_{8.1}}{\lambda^{s}}-\left(1+\lambda^{-\gamma}\right)\left(\frac{1+\varepsilon_{8.1}}{\lambda^{s}}\right)^{n}\right)\right] .
\end{aligned}
$$

The right part of (8.4) is less than $\left(1+\varepsilon_{8.1}\right) \lambda^{-s}$ for a suitable $\varepsilon_{8.1}=O\left(\lambda^{-r}\right), s>r>t$. 
We let $K_{n+1, m}=G_{n+1}^{-1}\left(K_{n+1}\right)$ correspond to $\delta_{n+1}^{-m}=G_{n+1}^{+1}\left(\delta_{n+1}\right)$. We have

$$
\frac{\operatorname{mes} \delta_{n+1}^{-m}}{\operatorname{mes} K_{n+1, m}}<\frac{\operatorname{mes} \delta_{n+1}}{\operatorname{mes} K_{n+1}} \cdot \exp \mu\left(G_{n+1}\right) \text {. }
$$

Because of $b_{4 n+1}$, the right side of (8.4) with the additional factor $\exp \mu\left(G_{n+1}\right)$ is still less than $\left(1+\varepsilon_{8.1}\right) \lambda^{-s}$ and (8.1) is proved for $k=n+1$. Lemma 2 with $\varepsilon=\varepsilon_{8.1}$ follows now from

$$
\operatorname{mes}\left(\bigcup \delta_{n+1}^{-m}\right)<(1+\varepsilon) \lambda^{-s} \cdot \operatorname{mes}\left(\bigcup K_{n, m}\right)<(1+\varepsilon) \lambda^{-s} \cdot \operatorname{mes}\left(\bigcup \delta_{n}^{-m}\right)<\left(\frac{1+\varepsilon}{\lambda^{s}}\right)^{n+1} .
$$

The estimates of Sects. 6-8 prove the following

Proposition 1. Let $\lambda \in\left[N_{0}, N_{0}+4\right]$ be so that for any $n=1,2, \ldots, F_{n+1}$ $\circ\left(\lambda, \frac{1}{2}\right) \in[0,1] \bigcup_{m=0}^{\infty}\left(\bigcup \hat{\delta}_{n}^{-m}\right)$. Then a partition $\xi_{\lambda}$ as in Sect. 1 exists.

Remark VIII/3. Notice that if $\lambda$ is such that at step $n F_{n-1} \circ h\left(\lambda, \frac{1}{2}\right)$ falls into a limit set $\mathscr{F}_{n}$ defined in Sect. 3 the condition of Proposition 1 will be satisfied. It is certainly so at Step $n$, and at subsequent steps the holes $\hat{\delta}_{p}^{-m}$ lie either in $\hat{\delta}_{n}^{-m}$, or in the intervals $F_{n-1} \circ g, \hat{f}_{n}$ constructed at Step $n$ (there is no middle branch $F_{p} \circ h$ for $p \geqq n$ ). The estimates of Sects. $6-8$ are even better in this case.

Remark VIII/4. If we suppose $F_{n-1} \circ h\left(\lambda, \frac{1}{2}\right)$ is outside $\hat{\delta}_{1}(\lambda)=\left(\frac{1}{2}-\lambda^{-(s-\alpha)}\right.$, $\left.\frac{1}{2}+\lambda^{-(s-\alpha)}\right)$ for all $n$, the above condition of Proposition 1 will be satisfied. In particular, if $h\left(\lambda, \frac{1}{2}\right)$ falls into some $f_{\lambda}$-invariant set (e.g. periodic orbit or invariant Cantor set of [5]) lying outside $\hat{\delta}_{1}(\lambda), \lambda$ satisfies this condition. Thus card $\{\lambda$ satisfying Proposition 1 \} equals the continuum. One can check however, using estimates of Sect. 11, that mes $\left\{\lambda: F_{n-1} \circ h\left(\lambda, \frac{1}{2}\right) \in[0,1] \backslash \hat{\delta}_{1}(\lambda)\right\}=0$.

\section{Velocities of Endpoints of Domains $\Delta f_{n}(\lambda)$}

Let $f_{n}$ be one of the maps constructed at step $n$, with domain $\Delta f_{n}=\left[x_{1 n}, x_{2 n}\right]$. In this section we prove the following

Lemma 3. There is an $\varepsilon=O\left(\lambda^{-s(1-v)}\right)$ such that for $i=1,2$

$$
\left|\frac{d x_{i n}(\lambda)}{d \lambda}\right|<\frac{\lambda^{s n}(1+\varepsilon)}{8 \lambda}
$$

Proof. Any $x_{i k}(\lambda)$ satisfies $f_{k}\left(\lambda, x_{i k}(\lambda)\right)=0$ or 1 . Thus

$$
\left|\frac{d x_{i k}(\lambda)}{d \lambda}\right|=\left|\frac{\partial f_{k}\left(\lambda, x_{i k}(\lambda)\right) / \partial \lambda}{\partial f_{k}\left(\lambda, x_{i k}(\lambda)\right) / \partial x}\right| \text {. }
$$

We proceed by induction as in the main construction. Consider the maps $f_{k}(2 \leqq k$ $\leqq n), G_{n}: \delta_{n}^{-m} \rightarrow \delta_{n}$, and $\hat{f}_{n}$. Assume inductively that the following estimates hold:

$$
\left.c_{1 k}\right)\left|\frac{\partial f_{k}(\lambda, x) / \partial_{\lambda}}{\partial f_{k}(\lambda, x) / \partial x}\right|<\frac{\lambda^{s k}}{8 \lambda}\left[1+\sum_{i=1}^{k-1} \frac{1+\varepsilon}{\lambda^{s(1-v) i}}\right]
$$


$\left.c_{2 n}\right)$ Let $H_{n}$ denote either $G_{n}$ or $\hat{f}_{n}$, and pick $p$ so that, if $p \leqq n$, then $\Delta H_{n} \subset[0,1] \backslash \delta_{p}$, while if $p>n$, then $\operatorname{dist}\left(\Delta H_{n}, \frac{1}{2}\right)>\lambda^{-s p}$. Let $N=\max (n, p)$. Then

$$
\left|\frac{\partial H_{n}(\lambda, x) / \partial \lambda}{\partial H_{n}(\lambda, x) / \partial x}\right|<\frac{\lambda^{s N}}{8 \lambda}\left[1+\sum_{i=1}^{n-1} \frac{1+\varepsilon}{\lambda^{s(1-v) i}}\right] \text {. }
$$

For $k=1$, these estimates are proven in Sect. 2. We will prove $c_{1 n+1}$ in the various cases that arise from the construction. $c_{2 n+1}$ is similar. In particular, $c_{1 n}$ implies Lemma 3.

Suppose $\varphi_{i}(\lambda, x), i=1, \ldots, n$ are $C^{1}$ functions, and define

$$
F(\lambda, x)=\varphi_{n}\left(\lambda, \varphi_{n-1}\left(\lambda, \ldots, \varphi_{1}(\lambda, x) \ldots\right)\right) .
$$

One sees that

$$
\frac{\partial F}{\partial \lambda}=\sum_{k=1}^{n}\left[\left(\frac{\partial \varphi_{k}}{\partial \lambda}\right) \prod_{i=k+1}^{n} \frac{\partial \varphi_{i}}{\partial x}\right]
$$

so that, at any point $\left(\lambda_{0}, x_{0}\right)$ in the domain of $F$,

$$
\frac{\partial F / \partial \lambda}{\partial F / \partial x}=\sum_{k=1}^{n}\left[\left(\frac{\partial \varphi_{k} / \partial \lambda}{\partial \varphi_{k} / \partial x}\right) \cdot\left(\prod_{i=1}^{k-1} \frac{\partial \varphi_{i}}{\partial x}\right)^{-1}\right],
$$

where the partials of $\varphi_{i}$ are evaluated at $\left(\lambda_{0}, \varphi_{i-1}\left(\lambda_{0}, \ldots, \varphi_{1}\left(\lambda_{0}, x_{0}\right) \ldots\right)\right)$.

To prove $c_{1 n+1}$, we first consider the case

Since

$$
f_{n+11}=f_{i_{n}} \circ f_{i_{n-1}} \circ \ldots \circ f_{i_{1}} \circ g_{n}(\lambda, x) \text {. }
$$

$$
g_{n}(\lambda, x)=\lambda x(1-x) \quad\left|x-\frac{1}{2}\right|>\lambda^{-s(n+1)}
$$

we have

$$
\left|\partial g_{n} / \partial \lambda\right|<\frac{1}{4},\left|\partial g_{n} / \partial x\right|>2 \lambda / \lambda^{s(n+1)} .
$$

Using (9.1), $a_{1 n}^{2}$ and $c_{1 k}\left(k=i_{1}, \ldots, i_{n}\right)$, we obtain

$$
\begin{aligned}
\left|\frac{\partial f_{n+11} / \partial \lambda}{\partial f_{n+11} / \partial x}\right|< & \frac{\lambda^{s(n+1)}}{2 \lambda}\left[\frac{1}{4}+\frac{\lambda^{s}(1+\varepsilon)}{8 \lambda}\left(1+\frac{1}{\lambda^{s(1-v)}-1}\right)\right. \\
& \left.\cdot\left(1+\frac{\lambda^{s}}{2 \lambda^{c_{0}}}+\ldots+\left(\frac{\lambda^{s}}{2 \lambda^{c_{0}}}\right)^{n-1}\right)\right] \\
< & \frac{\lambda^{s(n+1)}}{8 \lambda}\left[1+\frac{1+\varepsilon_{9.2}}{2 \lambda^{c_{0}}}\right] .
\end{aligned}
$$

This proves $c_{1 n+1}$ in case $f_{n+11}=f_{k} \circ F_{n-1} \circ g_{n}$.

In case $f_{n+11}=f_{k}^{\circ} \hat{f}_{n},(9.1), c_{1 k}$ and $c_{2 n}$, and $a_{3 n}$ imply

$$
\left|\frac{\partial f_{n+11} / \partial \lambda}{\partial f_{n+11} / \partial x}\right|<\frac{\lambda^{s(n+1)}}{8 \lambda}\left[1+\sum_{i=1}^{n-1} \frac{1+\varepsilon}{\lambda^{s(1-v) i}}+\frac{1}{\lambda^{y n}}\right] \text {. }
$$

Similarly, if $F_{n-1}=f_{i_{n-1}} \circ \ldots \circ f_{i_{1}}$ and $\left|x-\frac{1}{2}\right|>\lambda^{-s p}$, then

$$
\left|\frac{\partial\left(F_{n-1} \circ g\right) / \partial \lambda}{\partial\left(F_{n-1} \circ g\right) / \partial x}\right|<\frac{\lambda^{s p}}{8 \lambda}\left(1+\frac{1+\varepsilon_{9.4}}{2 \lambda^{c_{0}}}\right) .
$$


Now let $G_{n}^{\prime}=G_{n} \circ F_{n-1} \circ g$, where $\left.\Delta\left(F_{n-1} \circ g\right) \subset[0,1]\right)\left(\frac{1}{2}-\frac{1}{\lambda^{s p}}, \quad \frac{1}{2}+\frac{1}{\lambda^{s p}}\right)$, and $\Delta G_{n} \subset[0,1] \backslash \delta_{n}$. Using (9.1), $c_{2 n}, a_{2 n}$ and $a_{4 n}^{2}$, we see that

$$
\begin{aligned}
\left|\frac{\partial G_{n}^{\prime} / \partial \lambda}{\partial G_{n}^{\prime} / \partial x}\right| & <\frac{\lambda^{s p}}{8 \lambda}\left(1+\frac{1+\varepsilon_{9.4}}{2 \lambda^{c_{0}}}\right)+\frac{\lambda^{s n}(1+\varepsilon)}{(8 \lambda)(2 \lambda)^{c_{0}(n-1)}\left(2 \lambda^{1-s p}\right)} \sum_{i=0}^{n-1} \lambda^{-s(1-v) i} \\
& =\frac{\lambda^{s p}}{8 \lambda}\left(1+\frac{1+\varepsilon_{9.5}}{2 \lambda^{c_{0}}}\right) .
\end{aligned}
$$

On the other hand, for $G_{n}^{\prime}=G_{n} \circ \hat{f}_{n}$ where $\left.\Delta \hat{f}_{n} \subset[0,1]\right)\left(\frac{1}{2}-\frac{1}{\lambda^{s p}}, \frac{1}{2}+\frac{1}{\lambda^{s p}}\right)$, we obtain an estimate similar to $(9.3)$ :

$$
\left|\frac{\partial G_{n}^{\prime} / \partial \lambda}{\partial G_{n}^{\prime} / \partial x}\right|<\frac{\lambda^{s p}}{8 \lambda}\left(1+\sum_{i=1}^{n-1} \frac{1+\varepsilon}{\lambda^{s(1-v) i}}+\frac{1}{\lambda^{\gamma n}}\right) .
$$

Finally, let $\tilde{G}_{n}=G_{n}$ or $G_{n}^{\prime}, \Delta \tilde{G}_{n} \subset \delta_{n} \backslash \delta_{n+1}$. Then in $c_{2 n},(9.4)$ and (9.6), $p=n+1$. Now $a_{4 n}^{1}$ implies $\left|D \tilde{G}_{n}\right|>\lambda^{n s(1-v)}$. Hence using (9.1), (9.2) or (9.3) and $c_{2 n},(9.5)$ and (9.6), we obtain for $f_{n+1 k}=f_{n+11} \circ \tilde{G}_{n_{1}} \circ \ldots \circ \tilde{G}_{n_{k-1}}$

$$
\begin{aligned}
\left|\frac{\partial f_{n+1 k} / \partial \lambda}{\partial f_{n+1 k} / \partial x}\right|< & \frac{\lambda^{s(n+1)}}{8 \lambda}\left[1+\sum_{i=1}^{n-1} \frac{1+\varepsilon}{\lambda^{s(1-v) i}}+\frac{1}{\lambda^{\gamma n}}\right]\left[1+\frac{1}{\lambda^{s(1-v) n}}+\ldots\right. \\
& \left.+\frac{1}{\lambda^{s(1-v)(k-1) n}}\right] \\
& <\frac{\lambda^{s(n+1)}}{8 \lambda}\left[1+\sum_{i=1}^{n-1} \frac{1+\varepsilon}{\lambda^{s(1-v) i}}+\frac{1}{\lambda^{\gamma n}}\right]\left[1+\frac{1}{\lambda^{s(1-v) n}-1}\right] \\
& <\frac{\lambda^{s(n+1)}}{8 \lambda}\left[1+\sum_{i=1}^{n} \frac{1+\varepsilon}{\lambda^{s(1-v) i}}\right]
\end{aligned}
$$

for a suitable $\varepsilon=O\left(\lambda^{-s(1-v)}\right)$.

This proves $c_{1 n+1}$; the proof of $c_{2 n+1}$ is similar.

10. Construction of $\delta_{n+1}(\lambda)$ and $\hat{\delta}_{n+1}(\lambda)$. Structure of $\mathscr{X}_{n+1}$ in a $\lambda^{(-s+2 \alpha)(n+1)}$ Neighborhood of $\delta_{n+1}$

a) Recall that at step $n+1$ of the induction construction, we consider $\lambda$ contained in an interval $\mathscr{J}_{n}=\left[\lambda_{0 n}, \lambda_{1 n}\right]$. As $\lambda$ varies in $\mathscr{J}_{n}$, all the maps under consideration together with their domains vary continuously with $\lambda$.

The induction hypotheses $a_{i n}$ in Sect. 6 imply the following estimates on the diameters of the domains appearing at step $n+1$ :

$$
\left.\begin{array}{l}
\left|\Delta f_{k} \circ \hat{f}_{n}\right|<\lambda^{-s} \cdot\left(2 \lambda^{c_{1}}\right)^{-(n+1)} \\
\left|\delta_{n p_{0}}^{-m}\right|<\left|\delta_{n}\right| \cdot \lambda^{-s(1-v) p_{0}} \\
\left|\Delta f_{k} \circ F_{n-1} \circ g\right|<\left(2 \lambda^{\left.c_{1}\right)-(n+1)}\right.
\end{array}\right\}
$$


In the second estimate of (10.1), we write $\delta_{n}^{-m}$ as $\delta_{n p_{0}}^{-m}$, where $p_{0}$ denotes the minimum integer $p$ such that

$$
\delta_{n}^{-m} \subset\left[\frac{1}{2}-\lambda^{-s p}, \frac{1}{2}+\lambda^{-s p}\right] .
$$

In the third estimate, recall that $\Delta F_{n-1} \circ g=\left[x_{\min }, x_{\max }\right]$ with

$$
\left|x_{\min }-\frac{1}{2}\right|>\lambda^{-s(n+1)} \text {. }
$$

For any $G_{n}: \delta_{n}^{-k} \rightarrow \delta_{n}$ we have, according to $b_{4 n}$ of Sect. 6 , that $\mu\left(G_{n}\right)<\lambda^{-\alpha n}$. But actually for $\delta_{n}^{-k} \subset[0,1] \backslash \delta_{n+1}$, we can strongly enlarge $\delta_{n}^{-k}$ and still have the maps $G_{n}$ defined with $\mu\left(G_{n}\right)$ small. Let us consider the homothetic transformation

$$
\psi_{n}(\lambda): x \mapsto \frac{1}{2}+\left(x-\frac{1}{2}\right) \lambda^{2 \alpha n} .
$$

It follows from the condition $\alpha \leqq s / 4$ that for $n \geqq 3$ one can define

$$
q(n)=\max \left\{q: \psi_{n}(\lambda) \delta_{n}(\lambda) \subset \delta_{q}(\lambda)\right\} \geqq 1 .
$$

Remark $X / 1$. For $n=1$, the endpoints of $\psi_{1}(\lambda) \delta_{1}(\lambda)$ belong to $\bigcup \Delta f_{1}$. We define $\delta_{q(1)}(\lambda)$ for all $\lambda \in \mathscr{J}_{n}$ as the minimal interval containing $\psi_{1}(\lambda) \delta_{1}(\lambda)$ of the form $\left[x_{1 \max }(\lambda), x_{2 \max }(\lambda)\right]$, where $x_{1 \max }(\lambda) \in\left[0, \frac{1}{2}\right]$ and $x_{2 \max }(\lambda) \in\left[\frac{1}{2}, 1\right]$ are endpoints of domains $\Delta f_{1}$. We define $\delta_{q(2)}(\lambda)$ in an analogous way whenever $\psi_{2}(\lambda) \delta_{2}(\lambda) \not \subset \delta_{1}(\lambda)$.

It follows from the construction of Sect. 3 that for every interval $G_{n}^{-1} \delta_{n}$ (or $\left(G_{n}^{\prime}\right)^{-1} \delta_{n}$ ) which lies outside the domain $\Delta F_{q(n)} \circ$, the corresponding preimage $G_{n}^{-1} \delta_{q(n)}$ is defined. Indeed, the maps $G_{n}$ under consideration are those compositions of $G_{q(n)}$ and $F_{k} \circ g$ or $\hat{f}_{k}, q(n) \leqq k \leqq n$, which map their domains onto [0,1]. Using Lemma 1 and following the proof of $b_{4 n}$, we get for some $\varepsilon_{10.2}<\lambda^{-t}$

$$
\mu\left(G_{n}, \delta_{q(n)}^{-k}\right)<\left(1+\varepsilon_{10.2}\right) \lambda^{-\alpha q(n)} .
$$

From the definition of $q(n)$ for $n \geqq 3$ it follows that

$$
q(n) \geqq \max \left\{q:\left(1-2 \frac{\alpha}{s}\right) n>1\right\} .
$$

Since $2 \frac{\alpha}{S} \leqq \frac{1}{2}$, we get

$$
q(n) \geqq \max \left\{q: \frac{n}{2}>q\right\}=\left\{\begin{array}{lll}
\frac{n}{2}-1 & \text { for } & n \text { even } \\
\frac{n}{2}-\frac{1}{2} & \text { for } & n \text { odd }
\end{array}\right.
$$

In particular, we always have

$$
q(n) \geqq \frac{n}{2}-1 .
$$

We shall show that for $n \geqq 3$

$$
\Delta F_{q(n)} \circ h \subset\left(\frac{1}{2}-\frac{1}{\lambda^{s(n+1)}}, \frac{1}{2}+\frac{1}{\lambda^{s(n+1)}}\right) .
$$


Let $\Delta F_{q(n)} c h=\left[\frac{1}{2}-v_{1 n}, \frac{1}{2}+v_{2 n}\right], v_{i n}>0$. In a way analogous to (6.11) we get

$$
v_{i n}<1 /\left[(\sqrt{2})^{q(n)} \lambda^{\left(c_{0} q(n)+1\right) / 2}\right] .
$$

From (10.3) and (10.5) we obtain that for (10.4) it is enough to have $\frac{c_{0}}{2}\left(\frac{n}{2}-1\right)+\frac{1}{2}$ $>s(n+1)$, or taking into account that $c_{0}=1-s,\left(\frac{1-s}{4}-s\right) n>\frac{s}{2}$. This holds for $s \leqq \frac{1}{13}, n \geqq 1$.

From the fact that for $s \leqq \frac{1}{13}$ the domain of the central branch $\Delta h \subset \delta_{6}$, it follows that for $n \leqq 5$ if $G_{n}^{-1} \delta_{n} \subset \delta_{n} \backslash \delta_{n+1}$, then $G_{n}^{-1}[0,1]$ is defined.

In such a way, for all $n \geqq 1$ and for all domains

$$
\left.G_{n}^{-1} \delta_{n} \subset[0,1]\right)\left(\frac{1}{2}-\frac{1}{\lambda^{s(n+1)}}, \frac{1}{2}+\frac{1}{\lambda^{s(n+1)}}\right)
$$

the preimage

$$
G_{n}^{-1} \delta_{q(n)} \supset G_{n}^{-1}\left(\psi_{n}(\lambda) \delta_{n}(\lambda)\right)
$$

is defined.

b) Let us estimate the length of $\mathscr{J}_{n}$. When $\lambda$ varies in $\mathscr{J}_{n}$

$$
f_{i_{n-1}} \circ f_{i_{n-2}} \circ \ldots \circ f_{i_{1}} \circ h\left(\lambda, \frac{1}{2}\right)
$$

varies in one of $\Delta f_{i_{n}}$ and $f_{i_{n}} \circ f_{i_{n-1}} \ldots f_{i_{1}} \circ h\left(\lambda, \frac{1}{2}\right)$ varies in $[0,1]$. We have

$$
\frac{\partial\left(f_{i_{n}} \circ \ldots \circ f_{i_{1}} \circ h\left(\lambda, \frac{1}{2}\right)\right)}{\partial \lambda}=\prod_{k=1}^{n} \frac{\partial f_{i_{k}}}{\partial x}\left[\frac{1}{4}+\sum_{j=1}^{n} \frac{\partial f_{i_{j}} / \partial \lambda}{\partial f_{i_{i}} / \partial x}\left(\prod_{\ell=1}^{j-1} \frac{\partial f_{i_{\ell}}}{\partial x}\right)^{-1}\right],
$$

where the arguments of $f_{i_{p}}(\lambda, x)$ are $x=f_{i_{p-1}} \circ f_{i_{p-2}} \circ \ldots \circ f_{i_{1}} \circ h\left(\lambda, \frac{1}{2}\right)$. In consequence of $c_{1 k}$ and $a_{1 n}^{2}$, the sum in brackets is larger than $\frac{1}{4}-\frac{\left(1+\varepsilon_{10.6}\right) \lambda^{s}}{8 \lambda}>\frac{1}{4}\left(1-\lambda^{-c_{0}}\right)$. We shall use $v_{n}(\lambda)$ to denote the velocity of the top. We have

$$
v_{n}(\lambda)=\left|\frac{\partial\left(f^{n} \circ \ldots \circ f^{1} \circ h\left(\lambda, \frac{1}{2}\right)\right)}{\partial \lambda}\right|>\left(2 \lambda^{c_{0}}\right)^{n} \cdot \frac{1}{4}\left(1-\frac{1}{\lambda^{c_{0}}}\right) .
$$

Thus

$$
\left|\mathscr{J}_{n}\right|<4 \cdot\left(1-\lambda_{0 n}^{-c_{0}}\right)^{-1} \cdot\left(2 \lambda_{0 n}^{c_{0}}\right)^{-n} .
$$

We formulate the induction conditions on the choice of $\delta_{n}(\lambda)$.

i) The interval $\delta_{n}(\lambda)$ is of the form:

$$
\delta_{n}(\lambda)=\left(\frac{1}{2}-c_{n 1}(\lambda) \cdot \lambda^{-s n}, \frac{1}{2}+c_{n 2}(\lambda) \cdot \lambda^{-s n}\right), \quad 1 \leqq c_{n i}(\lambda)<1+o\left(\lambda^{-t n}\right) .
$$

ii) If for some $\delta_{n}^{-k} \delta_{q(n)}^{-k} \cap \delta_{n} \neq \emptyset$, then $\delta_{q(n)}^{-k} \subset \delta_{n}$.

iii) If $a_{n}$ is an endpoint of $\delta_{n}$, then $a_{n}$ coincides with a common endpoint of two intervals: some $\Delta f_{n}$ exterior to $\delta_{n}$ and some $\Delta F_{n-1} \circ g$ or $\Delta \hat{f}_{n}$ interior to $\delta_{n}$.

According to the construction of Sect. 3 we consider intervals $\Delta f_{k} \circ F_{n-1} \circ g$, $\Delta f_{k} \circ \hat{f}_{n},\left(G_{n}^{\prime}\right)^{-1} \delta_{n}$, and have to choose an interval $\delta_{n+1}(\lambda)$ satisfying the above conditions and varying continuously with $\lambda \in\left[\lambda_{0 n}, \lambda_{1 n}\right]$. 
Consider the point $\xi_{0 n}=\frac{1}{2}-\left(1+\lambda_{0 n}^{-\frac{\alpha}{8}(n+1)}\right) \lambda_{0 n}^{-s(n+1)}$. For $k=q(n)$, (ii) implies that if two intervals $\delta_{q(n)}^{-m}$ intersect, then one of them contains the other. Let $\bar{\delta}_{q(n)}^{-m}$ be the maximal interval containing $\xi_{0 n}$. Then we replace $\xi_{0 n}$ by $\xi_{1 n}$ which is the endpoint of $\bar{\delta}_{q(n)}^{-m}$. If $\xi_{0 n}$ is not contained in any $\delta_{q(n)}^{-m}$, but is inside some interval $\Delta f_{k} \circ F_{n-1} \circ \mathrm{g}$ or $\Delta f_{k}^{\circ} \circ \hat{f}_{n}$, we let $\xi_{1 n}$ be the right endpoint of this interval. If $\xi_{0 n}$ is a limit point of $\delta_{n}^{-m}$ we obtain any of the previous cases with an arbitrary small perturbation of $\xi_{0 n}$. The estimates $(10.1), c_{2 n},(10.8),(10.9)$ show that when $\lambda \geqq \lambda_{0 n}$ varies in $\mathscr{J}_{n}$

$$
\begin{aligned}
& \frac{1}{2}-\left(1+\lambda_{0 n}^{-\frac{\alpha}{8}(n+1)}\right) \cdot \lambda_{0 n}^{-s(n+1)}-\left(1+\varepsilon_{10.10}\right) \lambda_{0 n}^{-s}\left(2 \lambda_{0 n}^{c_{1}}\right)^{-(n+1)}<\xi_{1 n}(\lambda) \\
&<\frac{1}{2}-\left(1+\lambda_{0 n}^{-\frac{\alpha}{8}(n+1)}\right) \cdot \lambda_{0 n}^{-s(n+1)}+2\left(1+\varepsilon_{10.10}\right) \lambda_{0 n}^{-[s q(n)+s(1-v)] n} \\
&+\left(1+\varepsilon_{10.10}\right) \lambda_{0 n}^{-s}\left(2 \lambda_{0 n}^{c_{1}}\right)^{-(n+1)} .
\end{aligned}
$$

We shall show that for $n \geqq 7 \lambda_{0 n}^{-[s q(n)+s(1-v) n]} \ll \lambda_{0 n}^{-\left(\frac{\alpha}{8}+s\right)(n+1)}$. For this it is enough to have $s q(n)+s(1-v) n>\left(s+\frac{\alpha}{8}\right)(n+1)$. Since

$$
q(n) \geqq \frac{n}{2}-1, \quad \alpha \leqq \frac{s}{4}, \quad v=\frac{2(s-\alpha)}{1-s}<\frac{2 s}{1-s},
$$

we get the inequality $n\left(\frac{1}{2}-\frac{2 s}{1-s}-\frac{1}{32}\right)>2+\frac{1}{32}$, which holds for $n \geqq 7, s \leqq \frac{1}{13}$.

For $n \leqq 6$ the check that for $\delta_{q(n)}^{-1} \subset \delta_{n} \backslash \delta_{n+1},\left|\delta_{q(n)}^{-1}\right| \ll \frac{1}{\lambda^{s(n+1)}}$ is straightforward. The worst estimates correspond to $n=6$. Since $q(6) \geqq 2$ and $\left.D h\right|_{\delta_{6} \backslash \delta_{7}}>2 \lambda^{1-7 s}$, we get

$$
\left|h^{-1} \delta_{2}\right|<\frac{1+\varepsilon}{\lambda^{1-5 s}} \ll \frac{1}{\lambda^{7 s}}
$$

for $s \leqq \frac{1}{13}$.

Taking into account (10.8) and the formula $c_{1}=1-2 s \geqq 11 s$, we obtain from (10.10)

$$
\frac{1}{2}-\left(1+2 \cdot \lambda^{-\frac{\alpha}{8}(n+1)}\right) \lambda^{-s(n+1)}<\xi_{1 n}(\lambda)<\frac{1}{2}-\lambda^{-s(n+1)}
$$

and we can make $\xi_{1 n}$ the left endpoint of $\delta_{n+1}(\lambda)$. The analogous choice of the right endpoint gives us

$$
\delta_{n+1}(\lambda)=\left(\frac{1}{2}-\left(1+c_{n+11}\right) \lambda^{-s(n+1)}, \frac{1}{2}+\left(1+c_{n+12}\right) \lambda^{-s(n+1)}\right) c_{n+1 i}=o\left(\lambda^{-t(n+1)}\right) .
$$

One easily checks that $\delta_{n+1}(\lambda)$ also satisfies (ii) and (iii).

c) We then construct an enlarged interval $\hat{\delta}_{n+1}(\lambda)$. We begin by expanding $\delta_{n+1}\left(\lambda_{0 n}\right)$ with a homothetic transformation

$$
\varphi_{n+1}: x \rightarrow \frac{1}{2}+\left(x-\frac{1}{2}\right) \lambda_{0 n}^{\alpha(n+1)}\left(1+\lambda_{0 n}^{-\frac{\alpha}{8}(n+1)}\right) .
$$

Then we proceed with the endpoints of $\varphi_{n+1} \delta_{n+1}\left(\lambda_{0 n}\right)$ as above, i.e. using a small perturbation we make the endpoints of $\varphi_{n+1} \delta_{n+1}\left(\lambda_{0 n}\right)$ coincide with endpoints of 
some interval $\Delta f_{k}, k \leqq n$. One checks as above, that this can be done so that the interval $\hat{\delta}_{n+1}(\lambda)$ satisfies for all $\lambda \in \mathscr{J}_{n}=\left[\lambda_{0 n}, \lambda_{1 n}\right]$ the inequalities:

$$
\lambda^{\alpha(n+1)}\left|\delta_{n+1}(\lambda)\right|<\left|\hat{\delta}_{n+1}(\lambda)\right|<\lambda^{\alpha(n+1)}\left|\delta_{n+1}(\lambda)\right|\left(1+O\left(\lambda^{-\frac{\alpha}{8}(n+1)}\right)\right) .
$$

As $\left.\hat{\delta}_{n+1}(\lambda) \subset \delta_{q(n+1}\right)(\lambda)$, for any $\delta_{n+1}^{-k}(\lambda)=G_{n+1}^{-1} \delta_{n+1}(\lambda)$ the corresponding interval $\hat{\delta}_{n+1}^{-k}=G_{n+1}^{-1} \hat{\delta}_{n+1}^{q(\lambda)}$ is defined. Taking into account an additional factor $\exp \mu\left(G_{n+1}, \hat{\delta}_{n+1}^{-k}\right)<(1+\varepsilon) \lambda^{-(s-\alpha)(n+1)}$ we still have

$$
\lambda^{\alpha(n+1)}\left|\delta_{n+1}^{-k}(\lambda)\right|<\left|\hat{\delta}_{n+1}^{-k}(\lambda)\right|<\lambda^{\alpha(n+1)} \cdot\left(1+o\left(\lambda^{-t(n+1)}\right)\right) \cdot\left|\delta_{n+1}^{-k}(\lambda)\right|
$$

d) When estimating mes $\mathscr{M}_{n+1}$ we shall use the following

Lemma 4. For any $n$ there is a set $L_{n} \subset \mathscr{X}_{n}$ corresponding to $\delta_{n}$, and for any $\delta_{n}^{-k} \subset[0,1] \backslash \delta_{n}$ there is a corresponding set $\mathrm{L}_{n}^{-k} \subset \mathscr{X}_{n}$, such that

and

(a) if $\delta_{n}^{-k_{1}} \neq \delta_{n}^{-k_{2}}$ then $L_{n}^{-k_{1}} \cap L_{n}^{-k_{2}}=\emptyset$

(b) $\operatorname{mes}\left(L_{n}^{-k}\right)>\left(1-\varepsilon_{0}\right) \lambda^{2 \alpha n} \operatorname{mes}\left(\delta_{n}^{-k}\right)$, with $\varepsilon_{0}=O\left(\lambda^{-2 \alpha}\right)$.

Proof. In addition to the estimate $\alpha \leqq s / 4$, we will suppose that $\alpha$ has the form

$$
\alpha=s / 2 k_{0},
$$

where $k_{0}$ is an integer $\geqq 2$. This assumption is not really necessary, but it simplifies the notation.

If an interval $\delta$ with center $x_{0}$ and a number $c>0$ are given, we shall denote by $c \cdot \delta$ the image of $\delta$ under the homothetic transformation $x \rightarrow x_{0}+\left(x-x_{0}\right) \cdot c$. Further, we shall use $\delta_{n}^{(r)}$ to denote the set $\lambda^{2 \alpha r} \cdot \delta_{n} \backslash \lambda^{2 \alpha(r-1)} \cdot \delta_{n}$.

$$
\text { Let } \varepsilon_{1}=3 \cdot \lambda^{-2 \alpha}, \psi_{0}=0, \psi_{i}=\left(2 \cdot \lambda^{-(s-2 \alpha)}\right)^{i}, i \geqq 1, c_{n}=\prod_{i=0}^{n-1}\left(1+\psi_{i}\right), c=\lim _{n \rightarrow \infty} c_{n} \text {. }
$$

We prove Lemma 4 by induction. We assume that $L_{n}, L_{n}^{-k}$ are constructed and consist of $\Delta f_{r}, r \leqq n$, and that the following property holds : For any $\delta_{n}^{-k}, k=0,1, \ldots$ there exists an increasing sequence of intervals $\left(\lambda^{2 \alpha r} \cdot \delta_{n}\right)^{-k}, r=0,1, \ldots, R=R\left(\delta_{n}^{-k}\right)$ $\geqq n$, such that

$$
\frac{\operatorname{mes}\left(\lambda^{2 \alpha(r-1)} \cdot \delta_{n}\right)^{-k}}{\operatorname{mes}\left(L_{n}^{-k} \cap \delta_{n}^{(r)}\right)}<\frac{c_{n}\left(1+\varepsilon_{1}\right)}{\lambda^{2 \alpha}} .
$$

We define $L_{n+1}^{-m}$ corresponding to $\delta_{n+1}^{-m}$ and prove (10.14) for $n+1$. Then Lemma 4 follows with $1-\varepsilon_{0}=c^{-1} \cdot\left(1+\varepsilon_{1}\right)^{-1}$.

Consider $\lambda^{2 \alpha n} \cdot \delta_{n} \subset \delta_{q(n)}$. Condition ii and the construction of $\delta_{n+1}$ imply $\delta_{q(n)}^{-m} \subset \delta_{n} \backslash \delta_{n+1}$ for $\delta_{n}^{-m} \subset \delta_{n} \backslash \delta_{n+1}$. Considering maximal elements $\bar{\delta}_{q(n)}^{-m}$ among $\left\{\delta_{q(n)}^{-m} \subset \delta_{n} \backslash \delta_{n+1}\right\}$ and the corresponding diffeomorphisms $\bar{G}_{n}^{-m}$, we transmit the structure from $\lambda^{2 a n} \cdot \delta_{n}$ into each $\bar{\delta}_{q(n)}^{-m}$ and obtain that corresponding to any $\delta_{n}^{-m} \subset \delta_{n} \backslash \delta_{n+1}$ one can pick $L_{n}^{-m} \subset \delta_{q(n)}^{-m} \subset \delta_{n} \backslash \delta_{n+1}$ so that $L_{n}^{-m} \cap L_{n}^{-r}=\emptyset$, if $\delta_{n}^{-m} \neq \delta_{n}^{-r}$ and (10.14) multiplied by an additional factor $\exp \left(\lambda^{-\alpha q(n)}\right)$ holds for $L_{n}^{-m}$.

Let us consider the domain $V_{n+1}=\lambda^{2 \alpha n} \cdot \delta_{n} \backslash \lambda^{2 \alpha(n+1)} \cdot \delta_{n+1}$. Taking into account (10.9), (10.11), and $s=2 k_{0} \alpha, k_{0} \geqq 2$, we obtain

$$
\operatorname{mes}\left(V_{n+1} \triangle\left(\lambda^{2 \alpha n} \cdot \delta_{n} \backslash \lambda^{2 \alpha\left[n-\left(k_{0}-1\right)\right]} \cdot \delta_{n}\right)\right)=o\left(\lambda^{-(s-2 \alpha)(n+1)}\right) .
$$


Together with (10.14) this implies

$$
c_{n}\left(1+\varepsilon_{1}\right)\left(1+\chi_{1 n+1}\right) \cdot \operatorname{mes}\left(L_{n} \bigcap V_{n+1}\right)>\lambda^{2 \alpha} \cdot \operatorname{mes}\left(\lambda^{2 \alpha(n+1)} \cdot \delta_{n+1}\right)
$$

(here and below $\chi_{i n+1}=o\left(\lambda^{-t(n+1)}\right)$ ).

For $\delta_{n+1}^{-m} \subset \delta_{n}^{-m} \subset \delta_{q(n)}^{-m} \subset \delta_{n} \backslash \delta_{n+1}$ the corresponding set $V_{n+1}^{-m}$ is defined and

$$
c_{n}\left(1+\varepsilon_{1}\right)\left(1+\chi_{2 n+1}\right) \cdot \operatorname{mes}\left(L_{n}^{-m} \bigcap V_{n+1}^{-m}\right)>\lambda^{2 \alpha} \cdot \operatorname{mes}\left(\lambda^{2 \alpha(n+1)} \cdot \delta_{n+1}\right)^{-m} .
$$

We define $\bar{L}_{n+1}=\bigcup\left(L_{n}^{-m} \bigcap V_{n+1}^{-m}\right)$ where the sum is taken over all $L_{n}^{-m} \subset \delta_{n} \backslash \delta_{n+1}$. For any $r \geqq 1$ such that $\delta_{n} \supset \lambda^{2 \alpha r} \cdot \delta_{n+1}$ consider $\delta_{q(n)}^{-m} \subset \delta_{n+1}^{(r)}$ and corresponding $\left(\lambda^{2 \alpha(n+1)} \cdot \delta_{n+1}\right)^{-m}, V_{n+1}^{-m} \subset \delta_{q(n)}^{-m}$. Since the dimensions of $\delta_{q(n)}^{-m}$ are small compared to $\delta_{n+1}^{(r)}$ (see the proof of 10.10 above) we obtain from (10.16) that

$$
\frac{\operatorname{mes}\left(\lambda^{2 \alpha(r-1)} \cdot \delta_{n+1}\right)}{\operatorname{mes}\left(\bar{L}_{n+1} \cap \delta_{n+1}^{(r)}\right)}<\frac{1}{\lambda^{2 \alpha}} \cdot \frac{\left(1-\lambda^{-2 \alpha}\right)^{-1}}{1-\frac{c_{n} \cdot\left(1+\varepsilon_{1}\right)\left(1+\chi_{2 n}\right)}{\lambda^{2 \alpha}}} .
$$

Besides, for any $\delta_{n}^{-m} \subset \delta_{n} \backslash \delta_{n+1}$,

$$
\left(L_{n}^{-m} \bigcap\left[\left(\lambda^{2 \alpha n} \cdot \delta_{n}\right)^{-m} \backslash V_{n+1}^{-m}\right]\right)=\left(L_{n}^{-m} \bigcap\left(\lambda^{2 \alpha(n+1)} \cdot \delta_{n+1}\right)^{-m}\right) .
$$

All $L_{n}^{-m}$ consist of domains $\Delta \hat{f}_{n}$ and $\Delta\left(F_{n-1} \circ g\right)$. At Step $n+1$ when constructing $\Delta f_{n+11}$ we reproduce the structure from $[0,1] \backslash \delta_{n}$ on each $\Delta \hat{f}_{n}$ or $\Delta\left(F_{n-1} \circ g_{n}\right)$ using respectively $\hat{f}_{n}^{-1}$ or $\left(F_{n-1} \circ g_{n}\right)^{-1}$. We denote by $\left(\delta_{n}^{-m}\right)^{\prime},\left(\delta_{q(n)}^{-m}\right)^{\prime}$ the new preimages of $\delta_{n}, \delta_{q(n)}$ under $\hat{f}_{n}^{-1},\left(F_{n-1} \circ g_{n}\right)^{-1}$. The estimate of mes $\left[\bigcup_{m=0}^{\infty} \bigcup \delta_{n}^{-m}\right]$ from Sect. 8 together with the estimate of $\mu\left(G_{q(n)},[0,1] \backslash \delta_{n+1}\right)$ show that after excluding the set $\bigcup_{m=0}^{\infty} \bigcup\left(\delta_{q(n)}^{-m}\right)^{\prime}$ from each $\Delta \hat{f}_{n}$ or $\Delta\left(F_{n-1} \circ g_{n}\right)$ the measures of $\bar{L}_{n+1}$ and of any $L_{n}^{-m} \bigcap\left(\lambda^{2 \alpha(n+1)} \cdot \delta_{n+1}\right)^{-m}$ are multiplied by a factor larger than $1-\left(2 \cdot \lambda^{-(s-2 \alpha)}\right)^{n}$. This factor implies the passage from $\varepsilon_{n}$ to $\varepsilon_{n+1}$ in estimates $(10.14)$ for $\left(\lambda^{2 \alpha r}\right.$ $\left.\cdot \delta_{n+1}\right)^{-m}$. We let $\left(L_{n}^{-m}\right)^{\prime} \bigcap\left[\left(\lambda^{2 \alpha(n+1)} \delta_{n+1}\right)^{-m}\right]$ correspond to $\left(\delta_{n+1}^{-m}\right)^{\prime}$. Thus to each $\delta_{n+1}^{-m},\left(\delta_{n+1}^{-m}\right)^{\prime} \subset \delta_{n} \backslash \delta_{n+1}$ uniquely corresponds its $\lambda^{2 \alpha(n+1)}-$ enlargement which does not intersect $\bar{L}_{n+1} \backslash \bigcup\left(\delta_{q(n)}^{-m}\right)^{\prime}$. We now set

$$
L_{n+1}= \begin{cases}L_{n} & \text { outside } \delta_{n} \\ \bar{L}_{n+1} \backslash \bigcup\left(\delta_{q(n)}^{-m}\right)^{\prime} & \text { inside } \delta_{n} \backslash \delta_{n+1} .\end{cases}
$$

Notice that $L_{n+1} \bigcap \delta_{n} \backslash \delta_{n+1}$ consists of $\Delta f_{n+11}$. (10.17) together with the estimate of $\bigcup\left(\delta_{q(n)}^{-m}\right)^{\prime}$ gives

$$
\frac{\operatorname{mes}\left(\lambda^{2 \alpha(\mu-1)} \cdot \delta_{n+1}\right)}{\operatorname{mes}\left(L_{n+1} \bigcap \delta_{n+1}^{(\mu)}\right)}<\lambda^{-2 \alpha}\left(1+2.5 \cdot \lambda^{-2 \alpha}\right)
$$

and (10.14) follows for $\delta_{n+1}$. The maps $G_{n}^{-1}, G_{n}^{\prime-1}$ and their compositions transmit (10.18) on $\left(\delta_{n+1}^{(r)}\right)^{-m} C \delta_{n}^{-m} \backslash \delta_{n+1}^{-m}$ with an additional factor $\exp \lambda^{-\alpha q(n)}$. Joining it to the above estimate of

$$
\operatorname{mes}\left[\left(L_{n}^{-m} \bigcap\left(\lambda^{2 \alpha(n+1)} \cdot \delta_{n+1}\right)^{-m}\right) \backslash \bigcup\left(\delta_{q(n)}^{-m}\right)^{\prime}\right]
$$

finishes the proof of (10.14) and of Lemma 4. 
Remark $X / 2$. The above construction is similar to one used in Sect. 8 in order to estimate the measure of holes at Step $n+1$.

Remark $X / 3 . R$ which bounds $r$ in (10.14), may be much larger than $n$. For example, the construction implies that the consecutive $\lambda^{2 \alpha r}$-enlargements of $\delta_{n}$ are taken until we obtain the whole interval $[0,1]$.

\section{The Positivity of Measure}

Remember that at step $n+1$ we consider $\lambda \in \mathscr{J}_{n}=\left[\lambda_{0 n}, \lambda_{1 n}\right]$. As $\lambda$ varies in $\mathscr{J}_{n}$, $F_{n-1} \circ h\left(\lambda, \frac{1}{2}\right)$ traverses some $\Delta f_{n}$ and $f_{n} \circ F_{n-1} \circ h\left(\lambda, \frac{1}{2}\right)=F_{n} \circ h\left(\lambda, \frac{1}{2}\right)$ traverses $[0,1]$. The set $\mathscr{X}_{n+1}(\lambda)=\bigcup_{k=1}^{n+1} \Delta f_{k}$ is defined for all $\lambda \in \mathscr{J}_{n}$, and all the domains $\Delta f_{k}=\left(\Delta f_{k}\right) \lambda$ as well as the holes $\delta_{n+1}^{-m}(\lambda)$ and their enlargements $\hat{\delta}_{n+1}^{-m}(\lambda)$ vary continuously with $\lambda \in \mathscr{J}_{n}$. We then define $\mathscr{M}_{n+1} \cap \mathscr{J}_{n}$ as the set consisting of those $\lambda \in \mathscr{J}_{n}$ for which

$$
F_{n} \circ h\left(\lambda, \frac{1}{2}\right) \in \mathscr{X}_{n+1}(\lambda) \bigcup_{m=0}^{\infty} \bigcup \hat{\delta}_{n+1}^{-m}(\lambda)
$$

We saw in Sect. 10 that the velocity of the top satisfies

$$
v_{n}(\lambda)=\left|\frac{\partial}{\partial \lambda} F_{n} \circ h\left(\lambda, \frac{1}{2}\right)\right|>\left(2 \lambda^{c_{0}}\right)^{n}\left[4\left(1+\varepsilon_{11.1}\right)\right]^{-1} .
$$

At the same time the endpoints $x_{k}(\lambda)$ of $\Delta f_{k}(\lambda), k \leqq n+1$, move with velocities

$$
\left|\frac{d x_{k}}{d \lambda}\right|<\frac{\left(1+\varepsilon_{11.2}\right)}{8 \lambda} \cdot \lambda^{s(n+1)} .
$$

(11.1) and (11.2) imply that for any $\Delta f_{k}, k \leqq n+1$, the condition $F_{n} \circ h\left(\lambda, \frac{1}{2}\right) \in \Delta f_{k}(\lambda)$ defines an interval $\mathscr{J}\left(\Delta f_{k}\right) \subset \mathscr{J}_{n}$, as does the condition $F_{n} \circ h\left(\lambda, \frac{1}{2}\right) \in \hat{\delta}_{n+1}^{-m}(\lambda)$.

A priori the condition

$$
\operatorname{mes} \bigcup_{m=0}^{\infty} \bigcup \hat{\delta}_{n+1}^{-m}(\lambda)<\left[(1+\varepsilon) \lambda^{-(s-\alpha)}\right]^{n+1}
$$

does not imply the predominance of

$$
\left\{\lambda \in \mathscr{J}_{n}: F_{n} \circ h\left(\lambda, \frac{1}{2}\right) \in[0,1] \mid \bigcup_{m=0}^{\infty} \bigcup \hat{\delta}_{n+1}^{-m}(\lambda)\right\}
$$

in $\mathscr{J}_{n}$, and we have to do some additional estimates. In consequence of Lemma 4 for $k=n+1$, to any $\delta_{n+1}^{-k}=G_{n+1}^{-1} \delta_{n+1}$ there corresponds uniquely a set $L_{n+1}^{-k}$ $=G_{n+1}^{-1} L_{n+1} \subset \mathscr{X}_{n+1} \bigcap \delta_{q(n+1)}^{-k}$ such that

$$
\operatorname{mes} L_{n+1}^{-k}>(1-\varepsilon) \lambda^{2 \alpha(n+1)}\left|\delta_{n+1}^{-k}\right| .
$$
holds :

We define $\hat{L}_{n+1}^{-k}=\left(L_{n+1}^{-k} \backslash \hat{\delta}_{n+1}^{-k}\right)$. Thus for any $\lambda \in \mathscr{J}_{n}$ the following estimate

$$
\operatorname{mes} \hat{L}_{n+1}^{-k}>\left(1-\varepsilon_{11.3}\right) \lambda^{\alpha(n+1)}\left|\hat{\delta}_{n+1}^{-k}\right|, \quad k=0,1, \ldots, \varepsilon_{11.3}=O\left(\lambda^{-2 \alpha}\right)
$$


Let $\mathscr{J}=\mathscr{J}\left(\delta_{q(n+1)}^{-k}\right)=\left[\lambda_{0}, \lambda_{1}\right]$ be an interval on the $\lambda$-axis such that $F_{n} \circ h\left(\lambda, \frac{1}{2}\right) \in \delta_{q(n+1)}^{-k}$ when $\lambda \in \mathscr{J}$. Because of the definition of $q(n),\left|\delta_{q(n+1)}^{-k}\right|$ $<\left(1+o\left(\lambda^{-t(n+2)}\right)\right) \cdot \lambda^{2 \alpha(n+1)} \cdot \lambda^{2 s} \cdot\left|\delta_{n+1}^{-k}\right|$. Then the comparison of velocities (11.1) and (11.2) implies

$$
|\mathscr{J}|<\frac{4 \cdot \lambda_{0}^{2 \alpha(n+1)+2 s}}{\left(2 \lambda_{0}^{c_{0}}\right)^{n}}\left(1+\varepsilon_{11.4}\right) \cdot\left|\delta_{n+1}^{-k}\left(\lambda_{0}\right)\right| \text {. }
$$

When $\lambda$ passes $\mathscr{J}$, the measures of $\hat{\delta}_{n+1}^{-k}$ and $\hat{L}_{n+1}^{-k}$ vary in particular because of the variation of $\partial / \partial x\left(G_{n+1}^{-1}\right)$. We shall show this variation is small.

a)

Lemma 5. Let $\Gamma_{p}$ denote $\delta_{p}$ if $p \leqq n$, and $\left(\frac{1}{2}-\lambda^{-s p}, \frac{1}{2}+\lambda^{-s p}\right)$ if $p>n$. Let $F_{\lambda}(x)$ be one of the diffeomorphisms $G_{n}(\lambda, x), \hat{f}_{n}(\lambda, x)$, or $f_{n}(\lambda, x)$, and suppose $\Delta F_{\lambda}(x) \subset[0,1] \backslash \Gamma_{p}$. Let $F_{\lambda}^{-1}(z)$ be the inverse diffeomorphism, and let $N=N(F)$ be the number of iterations of the initial map $g_{\lambda}: x \mapsto \lambda x(1-x) \bmod 1$ corresponding to $F_{\lambda}$ (i.e., $F_{\lambda}$ $\left.=g_{\lambda}^{N}\right)$. Then

$$
\left|\frac{\partial}{\partial \lambda} \frac{\partial F_{\lambda}^{-1}}{\partial z}\right|<\frac{\lambda^{2 s p}}{8 \lambda} N\left|\frac{\partial F_{\lambda}^{-1}}{\partial z}\right| \sum_{i=0}^{n} \lambda^{-s i} .
$$

Proof. We proceed by induction. Assuming Lemma 5 holds for $k \leqq n$, we need to prove the corresponding estimates for $n+1$. We begin by estimating $\frac{\partial}{\partial \lambda} \frac{\partial \phi^{-1}}{\partial z}$ for a composition of maps. Let

$$
\phi(\lambda, x)=\varphi_{n} \circ \varphi_{n-1} \circ \cdots \circ \varphi_{1}(\lambda, x),
$$

where our notation is similar to that in the calculations for (9.1). Several applications of the chain rule give

$$
\frac{\partial}{\partial \lambda}\left(\partial \phi^{-1} / \partial z\right)=\left[\partial \phi^{-1} / \partial z\right] \sum_{i=1}^{n}\left[\frac{\partial / \partial \lambda\left(\partial \varphi_{i}^{-1} / \partial z\right)}{\partial \varphi_{i}^{-1} / \partial z}-\frac{\partial^{2} \varphi_{i} / \partial x^{2}}{\left(\partial \varphi_{i} / \partial x\right)^{2}} \frac{\partial\left(\varphi_{i+1}^{-1} \circ \ldots \circ \varphi_{n}^{-1}\right)}{\partial \lambda}\right],
$$

where as before the arguments of $\varphi_{i}$ and its derivatives are $\lambda$ and $\varphi_{i-1}{ }^{\circ} \ldots \circ \varphi_{1}(x)$ while those of $\varphi_{i}^{-1}$ are $\lambda$ and $\varphi_{i+1}^{-1} \circ \ldots{ }^{\circ} \varphi_{n}^{-1}(z), z=\phi(\lambda, x)$ (for $i=n, \varphi_{n}^{-1}=\varphi_{n}^{-1}(\lambda, z)$, and there is no second term in the brackets).

Let $F_{1}=f_{n-1} \circ \ldots \circ f_{1} \circ g, \Delta F_{1} \subset[0,1] \backslash \Gamma_{p}$, and let $N_{i}$ denote the number of iterations corresponding to $f_{i}$. The expression $\partial / \partial \lambda\left(\varphi_{i+1}^{-1}{ }^{\circ} \ldots \circ \varphi_{n}^{-1}\right)$ in (11.6) equals $\partial x_{i+1} / \partial \lambda$, where $x_{i+1}(\lambda)$ satisfies

$$
\varphi_{n} \circ \ldots \circ \varphi_{i+1}\left(\lambda, x_{i+1}(\lambda)\right)=z .
$$

In our case $\varphi_{1}=g, \varphi_{i+1}=f_{i} 1 \leqq i \leqq n-1$ and the estimates of Sect. 9 give

$$
\left|\partial / \partial \lambda\left(f_{i}^{-1} \circ \ldots \circ f_{n-1}^{-1}\right)(\lambda, z)\right|<\frac{1+\varepsilon_{1}}{8 \lambda} \lambda^{s i} .
$$

For $g=\lambda x(1-x)$ we have

$$
\frac{\partial}{\partial \lambda} \frac{\partial g^{-1}}{\partial z}=\frac{1}{2 \lambda^{2}\left(x-\frac{1}{2}\right)}, \quad \frac{-\partial^{2} g / \partial x^{2}}{(\partial g / \partial x)^{2}}=\frac{1}{2 \lambda\left(x-\frac{1}{2}\right)^{2}} .
$$


For $f_{i}$ we have by estimates $b_{2 n}$ of Sect. 6 that

$$
\frac{\partial^{2} f_{i} / \partial x^{2}}{\left(\partial f_{i} / \partial x\right)^{2}}<1+\varepsilon_{2}
$$

Thus, (11.5) and (11.6) give

$$
\begin{aligned}
\left|\frac{\partial}{\partial \lambda} \frac{\partial F_{1}^{-1}}{\partial z}\right| \leqq & \left|\frac{\partial F_{1}^{-1}}{\partial z}\right| \cdot\left[\left(\frac{\lambda^{s p}}{2 \lambda^{2}}+\frac{\lambda^{2 s p}}{2 \lambda}\left(1+\varepsilon_{1}\right) \frac{\lambda^{s}}{8 \lambda}\right)+\left(\frac{\lambda^{2 s}}{8 \lambda} N_{1}+\frac{\lambda^{2 s}}{8 \lambda}\left(1+\varepsilon_{1}\right)\left(1+\varepsilon_{2}\right)\right)\right. \\
& \left.+\ldots+\left(\frac{\lambda^{2 s(n-2)}}{8 \lambda} N_{n-2}+\frac{\lambda^{s(n-1)}}{8 \lambda}\left(1+\varepsilon_{1}\right)\left(1+\varepsilon_{2}\right)\right)+\frac{\lambda^{2 s(n-1)}}{8 \lambda} N_{n-1}\right]
\end{aligned}
$$

Let $F_{2}=G \circ F_{1}$, where $G=G_{n}: \Delta G \rightarrow \delta_{n}, \Delta G \subset[0,1] \backslash \delta_{n}$, and $N_{G}$ is the number of iterates for $G$. The estimates of Sect. 9 imply $\left|\frac{\partial G^{-1}(z, \lambda)}{\partial \lambda}\right|<\frac{\lambda^{s n}\left(1+\varepsilon_{1}\right)}{8 \lambda}$.

Because $\frac{\left|\partial^{2} F_{1} / \partial x^{2}\right|}{\left(\partial F_{1} / \partial x\right)^{2}}<v\left(F_{1}, \Delta\left(F_{1}\right)\right)$ for $x \in \Delta F_{1}$, we obtain using (4.7), $a_{2 n}$ and $b_{2 n}$ that

$$
\begin{gathered}
\frac{\left|\partial^{2} F_{1} / \partial x^{2}\right|}{\left(\partial F_{1} / \partial x\right)^{2}}<\left(1+O\left(\lambda^{-\gamma}\right)\right)\left(1+\frac{\lambda^{2 s p}}{\lambda^{s / 2}\left(2 \lambda^{c_{0}}\right)^{n+1}}\right) \\
\quad+\frac{\lambda^{2 s p}}{\lambda^{s / 2}\left(2 \lambda^{c 0}\right)^{n+1}}=\frac{\lambda^{2 s p}\left(1+\varepsilon_{3}\right)}{\lambda^{s / 2}\left(2 \lambda^{c 0}\right)^{n+1}}+\left(1+\varepsilon_{3}\right) .
\end{gathered}
$$

Using (11.6) for $F_{2}=G \circ F_{1}$ we have

$$
\begin{aligned}
\left|\frac{\partial}{\partial \lambda} \frac{\partial F_{2}^{-1}}{\partial z}\right|< & {\left[\left|\frac{\partial}{\partial \lambda} \frac{\partial F_{1}^{-1}}{\partial z}\right||| \frac{\partial F_{1}^{-1}}{\partial z} \mid+\left(\frac{\lambda^{2 s p}\left(1+\varepsilon_{3}\right)}{\lambda^{s / 2} \cdot\left(2 \lambda^{c 0}\right)^{n+1}}+\left(1+\varepsilon_{3}\right)\right)\right.} \\
& \left.\cdot \frac{\lambda^{s n}\left(1+\varepsilon_{1}\right)}{8 \lambda}+\frac{\lambda^{2 s n}}{8 \lambda} \cdot N_{G}\right] \cdot\left|\frac{\partial F_{2}^{-1}}{\partial z}\right| .
\end{aligned}
$$

Substituting (11.7) in (11.8) we obtain (11.5) for $G_{n}^{\prime}=G_{n} \circ F_{n-1} \circ g$ constructed at the beginning of step $n+1$ (we have besides an additional factor less than $\lambda^{-c_{0}}$ in the right part of (11.5)). The proof for $G_{n}^{\prime}=G_{n}{ }^{o} \hat{f}_{n}, \Delta \hat{f}_{n} \subset[0,1] \backslash \Gamma_{p}$, is analogous. Considering $p=n+1$ in (11.7) we obtain the assertion of Lemma 5 for $f_{n+11}$. Then we consider the compositions $f_{n+1 k}=f_{n+11}{ }^{\circ} \tilde{G}_{n_{k}} \circ \ldots \circ \bar{G}_{n_{1}}$. The induction hypotheses and the previous estimates give

$$
\left|\frac{\partial}{\partial \lambda} \frac{\partial G_{n_{i}}^{\prime-1}}{\partial z}\right|<\frac{\lambda^{2 s(n+1)}}{8 \lambda} \cdot N_{i}\left|\frac{\partial G_{n_{i}}^{-1}}{\partial z}\right| \cdot \sum_{i=0}^{n} \lambda^{-s i} .
$$

The estimates of Sect. 9 give

$$
\left|\frac{\partial\left(\tilde{G}_{n_{l}}^{-1} \circ \ldots \circ \tilde{G}_{n_{k}}^{-1} \circ f_{n+11}^{-1}\right)}{\partial \lambda}\right|<\frac{\lambda^{s(n+1)}}{8 \lambda}\left(1+\varepsilon_{1}\right) .
$$

Taking into account

$$
\left|\frac{\partial^{2} G_{n_{2}}}{\partial x^{2}} /\left(\frac{\partial G_{n_{2}}}{\partial x}\right)^{2}\right|<\left(1+\varepsilon_{2}\right) \lambda^{-\alpha n},
$$


(11.6) implies

$$
\begin{aligned}
\left|\frac{\partial}{\partial \lambda} \frac{\partial f_{n+1 k}^{-1}}{\partial z}\right|< & {\left[\sum_{i=1}^{k}\left(\frac{\lambda^{2 s(n+1)}}{8 \lambda} \cdot N_{i} \cdot \sum_{i=0}^{n} \lambda^{-s i}+\frac{\left(1+\varepsilon_{2}\right)\left(1+\varepsilon_{1}\right) \cdot \lambda^{s(n+1)}}{\lambda^{\alpha n} \cdot 8 \lambda}\right)\right.} \\
& \left.+\frac{\lambda^{2 s(n+1)}}{8 \lambda} \cdot N\left(f_{n+11}\right)\right]\left|\frac{\partial f_{n+1 k}^{-1}}{\partial z}\right| .
\end{aligned}
$$

This proves Lemma 5 for $f_{n+1}$, and the sum in round brackets gives the desired estimate for $G_{n+1}$. The proof for $\hat{f}_{n+1}=f_{n+1} \circ G_{n}$ is similar.

b) Consider $\mathscr{J}\left(\delta_{q(n+1)}\right)=\left\{\lambda: F_{n} \circ h\left(\lambda, \frac{1}{2}\right) \in \delta_{q(n+1)}(\lambda)\right\}=\left[\lambda_{0}, \lambda_{1}\right]$. (11.4) gives $\left|\mathscr{J}\left(\delta_{q(n+1)}\right)\right|<\frac{\left(1+\varepsilon_{11.4}\right) \cdot 16 \cdot \lambda_{0}^{1+s}}{\left(2 \cdot \lambda_{0}^{c_{0}+s-2 \alpha}\right)^{n+1}}$. Let $\Delta(\lambda)$ be any interval in $L_{n+1} \bigcap \delta_{q(n+1)}$. The comparison of velocities (11.1) and (11.2) shows that the time it takes for $F_{n} \circ h\left(\lambda, \frac{1}{2}\right)$ to traverse $\Delta(\lambda)$ equals $\frac{|\Delta(\lambda)|}{v_{n}(\lambda)} \cdot\left(1+o\left(\lambda^{-c_{0} n}\right)\right)$, where $\lambda$ is any moment of passing by. We want to reduce all these moments (for different $\Delta(\lambda)$ ) to the same one, namely to $\lambda_{0}$, and then use the relation (11.3) for $\lambda_{0}$. This can be done for given $\Delta(\lambda)$ if for any $\lambda \in \mathscr{J}\left(\delta_{q(n+1)}\right)$,

$$
\frac{|\Delta(\lambda)|}{\left|\Delta\left(\lambda_{0}\right)\right|}>\left(1-\alpha_{n+1}\right), \quad \alpha_{n+1}=o\left(\lambda_{0}^{-t(n+1)}\right) .
$$
imply

Let $N=N\left(\Delta f_{k}\right)=N\left(f_{k}\right)$. If $N<\lambda_{0}^{s(n+1)}$, Lemma 5 and the estimate of $\mathscr{J}\left(\delta_{q(n+1)}\right)$

$$
\left|\Delta\left(\lambda_{0}\right)\right|-|\Delta(\lambda)|<\frac{\lambda_{0}^{2 s(n+1)}}{8 \lambda_{0}} \cdot \lambda_{0}^{s(n+1)} \cdot \frac{16 \cdot \lambda_{0}^{1+s} \cdot\left(1+\varepsilon_{11.9}\right)}{\left(2 \lambda_{0}^{c_{0}+s-2 \alpha}\right)^{n+1}} \cdot\left|\Delta\left(\lambda_{0}\right)\right| .
$$

Thus for such $\Delta, \alpha_{n+1}=O\left(\lambda_{0}^{\left[c_{0}-2(s+\alpha)\right](n+1)}\right)$.

Lemma 7 of Sect. 12 gives the following relation between $N(\Delta)$ and $|\Delta|$ for $\Delta \in[0,1] \backslash \delta_{n}$ :

$$
N<\frac{\sqrt{n} \cdot 2 s}{c_{0}}\left|\log _{\lambda_{0}}\right| \Delta||
$$

Thus $N<\lambda_{0}^{s(n+1)}$, if $\frac{2 s}{c_{0}} \sqrt{n+1}\left|\log _{\lambda_{0}}\right| \Delta||<\lambda_{0}^{s(n+1)}$. Lemma 7 also gives the following estimate for a domain $\Delta\left(F_{n-1} \circ h\right)$ of the central branch $F_{n-1} \circ h(\lambda, x)$. If $\Gamma_{p} \supset \Delta\left(F_{n-1} \circ h\right) \supset \Gamma_{p+1}$, and $N=N\left(F_{n-1} \circ h\right)=N\left(F_{n+1}\right)+1$, then

$$
N<\frac{4 s}{c_{0}} \sqrt{n} \cdot p
$$

When constructing $L_{n+1}$ in Sect. 10, we had $L_{n+1} \bigcap\left(\delta_{n} \backslash \delta_{n+1}\right) \subset \bigcup \Delta f_{n+11}$. Using this fact one can check inductively following the proofs of Lemmas 2 and 4 that the following construction gives a set $\mathscr{X}_{n+1}^{\prime} \subset \mathscr{X}_{n+1}$ with

$$
\operatorname{mes}\left(L_{n+1}^{-k} \bigcap \mathscr{X}_{n+1}^{\prime}\right)>\left(1-\varepsilon_{0}^{\prime}\right) \lambda^{2 \alpha(n+1)} \operatorname{mes} \delta_{n+1}^{-k}
$$

for every $\delta_{n+1}^{-k}$. 
We begin by constructing at step 2 the maps $f_{1} \circ g$ and the holes $g^{-1} \delta_{1}$. Then at step $n+1, n \geqq 2$, we reproduce on each interval inside $\delta_{n}$ the structure obtained after step $n$ on $[0,1] \backslash \delta_{n}$, and on each hole $\delta_{i}^{-k}\left[1 \leqq i \leqq n-1\right.$ and $k \leqq k_{0}(n)$ here, contrary to $i=n$ and $1 \leqq k<\infty$ in the construction of Sect. 3] we reproduce the structure of $\delta_{i} \backslash \delta_{n}$ obtained after step $n$. Ignoring $N\left(F_{n-1} \circ h\right)$ this construction gives for $N\left(\mathscr{X}_{n}^{\prime}\right)=\max \left\{N\left(\Delta f_{k}\right), \Delta f_{k} \in \mathscr{X}_{n}^{\prime}\right\}$ the upper estimate $2^{n}$. Taking into account $N\left(F_{n-1} \circ h\right)$ estimated above, we obtain

$$
N\left(\mathscr{X}_{n}^{\prime}\right)<n \cdot 2^{n}
$$

This implies the following

\section{Lemma 6.}

$$
\frac{\operatorname{mes}\left\{\Delta\left(f_{k}\right) \in \hat{L}_{n+1}(\lambda): N\left(f_{k}\right)<n \cdot 2^{n}\right\}}{\operatorname{mes} \hat{\delta}_{n+1}(\lambda)}>(1-\varepsilon) \lambda^{\alpha(n+1)} .
$$

Lemma 6 implies the predominance of $\Delta$ satisfying (11.9) in $\hat{L}_{n+1}$. Thus (11.3) implies

$$
\frac{\operatorname{mes}\left\{\lambda \in \mathscr{J}\left(\delta_{q(n+1)}\right), F_{n} \circ h\left(\lambda, \frac{1}{2}\right) \in \hat{L}_{n+1}(\lambda)\right\}}{\operatorname{mes}\left\{\lambda \in \mathscr{J}\left(\delta_{q(n+1)}\right), F_{n} \circ h\left(\lambda, \frac{1}{2}\right) \in \hat{\delta}_{n+1}(\lambda)\right\}}>\left(1-\varepsilon_{11.10}\right) \lambda_{0}^{\alpha(n+1)} .
$$

c) Let $\mathscr{J}=\mathscr{J}\left(\delta_{q(n+1)}^{-k}\right)=\left[\lambda_{2}, \lambda_{3}\right]=\left\{\lambda: F_{n} \circ h\left(\lambda, \frac{1}{2}\right) \in \delta_{q(n+1)}^{-k}(\lambda)\right\} .(11.4)$ and Lemma 5 imply that for any $\lambda \in \mathscr{J}$

$$
\begin{aligned}
& \left\|\left.\frac{\partial G_{n+1 \lambda}^{-1}}{\partial z}\right|_{z=1 / 2}-\left.\frac{\partial G_{n+1 \lambda_{2}}^{-1}}{\partial z}\right|_{z=1 / 2}\right\| \\
& \quad<\frac{\left(\left.\frac{\partial G_{n+1 \lambda_{2}}^{-1}}{\partial z}\right|_{z=1 / 2}\right)^{2} \cdot N\left(G_{n+1}\right) \cdot 2 \lambda_{2}^{s}\left(1+\varepsilon_{11.11}\right)}{\left(2 \lambda_{2}^{c_{0}-(s+2 \alpha)}\right)^{n+1}} .
\end{aligned}
$$

(11.11) and the estimate $b_{4 n+1}$ of $\mu\left(G_{n+1}\right)$ give for any $\Delta(\lambda) \subset\left(\hat{L}_{n+1} \cap \mathscr{X}_{n+1}^{\prime}\right)^{-k}$

$$
\begin{aligned}
\frac{\operatorname{mes} G_{n+1 \lambda}^{-1} \Delta(\lambda)}{\operatorname{mes} G_{n+1 \lambda_{2}}^{-1} \Delta\left(\lambda_{2}\right)}>\left(1-\alpha_{n+1}\right)\left(1-\frac{\left|\frac{\partial G_{n+1 \lambda}^{-1}}{\partial z}-\frac{\partial G_{n+1 \lambda_{2}}^{-1}}{\partial z}\right|_{z=1 / 2}}{\left|\frac{\partial G_{n+1 \lambda_{2}}^{-1}}{\partial z}\right|_{z=1 / 2}}\right) \\
\\
\cdot \exp \left(\mu\left(G_{n+1}\right)\right)>\left(1-\frac{N\left(G_{n+1}\right) \cdot\left|\frac{\partial G_{n+1 \lambda_{2}}^{-1}}{\partial z}\right| 2 \lambda_{2}^{s}\left(1+\varepsilon_{11.12}\right)}{\left(2 \lambda_{2}^{c_{0}-(s+2 \alpha)}\right)^{(n+1)}}\right) \\
\cdot\left(1-\alpha_{n+1}\right)\left(1-O\left(\lambda^{-\alpha(n+1)}\right) .\right.
\end{aligned}
$$

As $N(G) \cdot\left|\frac{\partial G^{1}}{\partial z}\right|=o(1)$, we obtain from (11.12) and (11.3)

$$
\frac{\operatorname{mes}\left\{\lambda \in \mathscr{J}\left(\delta_{q(n+1)}^{-k}\right), F_{n} \circ h\left(\lambda, \frac{1}{2}\right) \in \hat{L}_{n+1}^{-k}\right\}}{\operatorname{mes}\left\{\lambda \in \mathscr{J}\left(\delta_{q(n+1)}^{-k}\right), F_{n} \circ h\left(\lambda, \frac{1}{2}\right) \in \hat{\delta}_{n+1}^{-k}\right\}}>\left(1-\varepsilon_{11.13}\right) \cdot \lambda_{2}^{\alpha(n+1)}
$$

Using $\hat{L}_{n+1}^{-k}(\lambda) \bigcap \hat{L}_{n+1}^{-\ell}(\lambda)=\emptyset$, if $\hat{\delta}_{n+1}^{-k} \neq \hat{\delta}_{n+1}^{-\ell}$, we obtain from (11.10) and (11.13) 
Proposition 2. Let $\mathscr{J}_{n}=\left[\lambda_{0 n}, \lambda_{1 n}\right] \subset \mathscr{M}_{n}$ be any interval on the $\lambda$-axis constructed at Step $n$. Then

$$
\frac{\operatorname{mes}\left\{\lambda \in \mathscr{J}_{n}, F_{n} \circ h\left(\lambda, \frac{1}{2}\right) \in \bigcup_{k=0}^{\infty} \bigcup \hat{\delta}_{n+1}^{-k}(\lambda)\right\}}{\operatorname{mes} \mathscr{J}_{n}}<\frac{1+\varepsilon_{11}}{\lambda_{0 n}^{\alpha(n+1)}}
$$

where $\varepsilon_{11}<\lambda^{-t}$.

We define

$$
\mathscr{M}_{n+1} \bigcap \mathscr{J}_{n}=\left\{\lambda: F_{n} \circ h\left(\lambda, \frac{1}{2}\right) \in[0,1] \mid \bigcup_{k=0}^{\infty} \bigcup \hat{\delta}_{n+1}^{-k}(\lambda)\right\},
$$

and obtain

$$
\operatorname{mes}\left(\mathscr{M}_{n+1} \bigcap \mathscr{J}_{n}\right)>1-\frac{1+\varepsilon_{11}}{\lambda_{0 n}^{\alpha(n+1)}}
$$

and consequently

$$
\operatorname{mes} \mathscr{M}_{n+1}>\left(1-\frac{1+\varepsilon_{11}}{N_{0}^{\alpha(n+1)}}\right) \operatorname{mes} \mathscr{M}_{n} \text {. }
$$

Remark $X I / 1$. Any $\lambda$ such that $F_{n} \circ h\left(\lambda, \frac{1}{2}\right) \in[0,1] \mid \bigcup_{k=0}^{\infty} \bigcup \hat{\delta}_{n+1}^{-k}(\lambda)$ lies in one of the intervals $\mathscr{J}_{n+1}\left(\Delta_{k}\right)$ corresponding to the relation $F_{n} \circ h\left(\lambda, \frac{1}{2}\right) \in \Delta_{k}(\lambda)$, or is a limit point of such intervals. One can apparently prove that

$$
\operatorname{mes}\left\{\lambda: F_{n} \circ h\left(\lambda, \frac{1}{2}\right) \in \bigcup \Delta_{k}(\lambda)\right\}>\left(1-\frac{1+\varepsilon_{11}^{\prime}}{\lambda_{0 n}^{t(n+1)}}\right) \operatorname{mes} \mathscr{J}_{n},
$$

but there is no reason to avoid $\lambda$ lying in the limit set. They are even better in some sense (see Remark VIII/3).

\section{Transition from $T_{\lambda}$-Invariant Measure to $f_{\lambda}$-Invariant Measure}

The previous relations between mes $\mathscr{M}_{n+1}$ and mes $\mathscr{M}_{n}$, and the choice of the position of the top

$$
F_{n-1} \circ h\left(\lambda, \frac{1}{2}\right) \in[0,1]-\left(\frac{1}{2}-\frac{1}{\lambda^{s / 2}}, \frac{1}{2}+\frac{1}{\lambda^{s / 2}}\right)
$$

within the first steps $1,2, \ldots, n_{0}$, imply that there exists a set $\mathscr{M}=\bigcap_{n=1}^{\infty} \mathscr{M}_{n}$ on the $\lambda$-axis with measure

$$
\operatorname{mes} \mathscr{M}>4\left[\prod_{n=1}^{n_{0}}\left(1-\frac{2(1+\varepsilon)}{N_{0}^{s / 2}}\right)\right] \cdot\left[\prod_{n=n_{0}+1}^{\infty}\left(1-\frac{1+\varepsilon}{N_{0}^{\alpha n}}\right)\right]
$$

such that for any $\lambda \in \mathscr{M}$ the partition $\xi_{\lambda}$ of Sect. 1 exists. 
Conditions i-iv of Sect. 1 imply that for $T_{\lambda}$ defined by $T_{\lambda} \mid \Delta_{i}(\lambda)=f_{\lambda}^{n_{2}}$ there exists a unique $T_{\lambda}$-invariant probabilistic measure $v_{\lambda}<d x$ with a density $\varrho_{\lambda}(x) \in C_{[0,1]}, \varrho_{\lambda}$ $>c>0$. The endomorphism $T_{\lambda}$ of the Lebesgue space $\left([0,1], v_{\lambda}\right)$ is exact and its natural extension is isomorphic to a Bernouli shift (see $[12,13])$.

In order to finish the proof of Theorem $\mathrm{A}$ for the family $f_{\lambda}: x \rightarrow \lambda x(1-x)$ $(\bmod 1)$ we have to construct an invariant measure $\mu_{\lambda}<d x$.

Let $f^{-k}(A)$ be the full preimage of $A \subset[0,1]$ under $f^{k}, f^{-k} A=\left\{x: f^{k} x \in A\right\}$. Suppose $\sum_{\Delta_{i} \in \xi_{\lambda}} n_{i} v_{\lambda}\left(\Delta_{i}\right)<\infty$. Then the measure defined for any $d x$-measurable set $A$ by

$$
\mu_{\lambda}(A)=\sum_{\Delta_{i} \in \xi_{\lambda}} \sum_{0 \leqq j<n_{i}} v_{\lambda}\left(f^{-j} A \bigcap \Delta_{i}\right)
$$

is absolutely continuous with respect to $d x$, by a theorem on integrability of a series of positive functions (see for example [15] Sect. 14).

We show $\mu_{\lambda}$ is $f$-invariant.

By definition

$$
\mu_{\lambda}\left(f^{-1} A\right)=\sum_{\Delta_{i} \in \xi_{\lambda}} \sum_{0 \leqq j<n_{i}} v_{\lambda}\left(f^{-j} \circ f^{-1} A \bigcap \Delta_{i}\right) .
$$

If $j<n_{i}-1$, every term $f^{-j} \circ f^{-1} A \bigcap \Delta_{i}$ in (12.2) coincides with $f^{-(j+1)} A \bigcap \Delta_{i}$ in (12.1). After excluding these terms, there remain in (12.1) terms with $j=0$, which give $\sum_{\Delta_{i} \in \xi_{\lambda}} v_{\lambda}\left(A \bigcap \Delta_{i}\right)=v_{\lambda}(A)$, and in (12.2) terms with $j=n_{i}-1$, which give

$$
\sum_{\Delta_{i} \in \xi} v_{\lambda}\left(f^{-n_{2}} A \bigcap A\right)=\sum_{\Delta_{i} \in \xi} v_{\lambda}\left(T_{\lambda}^{-1} A \bigcap \Delta_{i}\right)=v_{\lambda} T_{\lambda}^{-1} A
$$

Thus (12.1) equals (12.2) because of the $T_{\lambda}$-invariance of $v_{\lambda}$.

Let $\beta=\frac{2}{5}$. The following proposition implies $\sum n\left(\Delta_{i}\right)\left|\Delta_{i}\right|<\infty$.

Proposition 3. $\sum_{\Delta_{i} \in \delta_{n} \backslash \delta_{n-1}} n\left(\Delta_{i}\right)\left|\Delta_{i}\right|<\frac{n^{3 / 2}}{\lambda^{s(1-\beta) n}}$.

Proof. a) Consider step $n$ of the induction construction of Sect. 3. If $\Phi_{n}$ is one of $f_{n}, \hat{f}_{n}$, $G_{n}, F_{n-1}$ obtained with $N$ successive iterates of $\mathrm{f}_{\lambda}$, we use an upper index so that $\Phi_{n}^{N}=f_{\lambda} \circ f_{\lambda} \circ \cdots \circ f_{\lambda}$, and $\Phi_{n}^{-N}=\left(\Phi_{n}^{N}\right)^{-1} \mid \operatorname{Im} \Phi_{n}^{N}$.

Lemma 7. $\left|D f_{n}^{N}\right|>\lambda^{\frac{c_{0} N}{2 \sqrt{n}}+s}$

Let

$$
\Gamma_{\ell}= \begin{cases}\delta_{\ell} & \text { if } \ell \leqq n \\ {\left[\frac{1}{2}-\lambda^{-s \ell}, \frac{1}{2}+\lambda^{-s \ell}\right]} & \text { if } \ell \geqq n+1 .\end{cases}
$$

We prove Lemma 7 by induction and assume that for $k=1, \ldots, n$ Lemma 7 holds together with the following properties:

i) Let $\delta_{n}^{-N}=G^{-N} \delta_{n} \subset[0,1] \backslash \Gamma_{\ell}$, and let $r=\max (1, n)$. Then $\left|D G^{N}\right| \delta_{n}^{-N} \mid>2 \frac{c_{0} N}{2 \sqrt{r}}+s$.

ii) Let $\Delta \hat{f}_{n}^{N} \subset[0,1] \backslash \Gamma_{\ell}$. Then $\left|D \hat{f}_{n}^{N}\right|>\lambda \frac{c_{0}}{2} \frac{N}{\sqrt{\ell}}$. 
Consider $k=n+1$. Notice that if $x \in[0,1] \backslash \Gamma_{2}$, then $\left|D f_{\lambda}\right|>\lambda^{1-2 s}>\lambda^{\frac{c_{0}}{2}}+s$. If $x \in \Gamma_{2}$, then $\ell, r \geqq 3$ in i), ii) and $\frac{c_{0}}{2 \sqrt{3}}+s<\frac{c_{0}}{2}$. As $\left|D f_{\lambda}\right|>\lambda^{\frac{c_{0}}{2}}$ on any hole $\delta_{1}^{-1}$ we obtain i) for $n=1$. ii) for $n=1$ holds because of i) and $\left|D f_{1}\right|>\lambda^{c_{0}}>\lambda^{\frac{c_{0}}{2}}+s$.

Let $F_{n-1} \circ h(\lambda, x)$ be the central branch, $F_{n-1}=f_{i_{n-1}} \circ \ldots \circ f_{i_{1}}, 1 \leqq i_{k} \leqq k, N\left(f_{i_{k}}\right)$ $=N_{k}$ the number of iterations of $f_{\lambda}$ corresponding to $f_{i_{k}}, k \in[1, n-1], \delta=G^{-N_{n}} \delta_{n}$, $M=1+\sum_{k=1}^{n} N_{k}$. Then $\left(F_{n-1} \circ h\right)^{-1} \delta=\delta_{n}^{-M}$. (In the notation of Sect. 3, $G^{M}: \delta_{n}^{-M} \rightarrow \delta_{n}$ is one of the $G_{n}^{\prime}$ constructed at the beginning of step $n$.)

Let $D_{n-1}$ be the domain of $F_{n-1} \circ$, and let $p_{0}=\min \left\{p \mid \delta_{n}^{-M} \subset[0,1] \backslash \Gamma_{p}\right\}$. Then (see $(6.12))$

$$
p_{0}>\frac{c_{0}}{2 s} n
$$

According to the construction of Sect. 3,

$$
\operatorname{dist}\left(\delta, F_{n-1} \circ h\left(\lambda, \frac{1}{2}\right)\right)>\frac{\delta}{2} \lambda^{\alpha n}(1-\varepsilon),
$$

which implies (see (6.4)) that

$$
\left|D G^{M}\right|>\frac{\sqrt{\lambda}\left[\left|D G^{N_{n}}\right| \prod_{k=1}^{n-1}\left|D f_{i_{k}}\right|\right]^{1 / 2}}{\lambda^{(s-\alpha) n / 2}},
$$

where $D G^{M}$ is evaluated on $\delta_{n}^{-M}$ and $D G^{N_{n}}$ on $\delta$. By the induction hypotheses we have

$$
\begin{aligned}
\left|D f_{i k}\right| & >\lambda^{\left[c_{0} N_{k} / 2 V \bar{k}\right]+s} \quad 1 \leqq k \leqq n-1 \\
\left|D G^{N_{n}}\right| & >\lambda^{\left[c_{0} N_{n} / 2 \sqrt{n}\right]+s} .
\end{aligned}
$$

Hence, on $\delta_{n}^{-M}$,

$$
\left|D G^{M}\right|>\lambda^{\theta}
$$

where

$$
\theta=\frac{1}{2}+\frac{s(n-1)}{2}+\left(\sum_{i=1}^{n} N_{i}\right) \frac{c_{0}}{4 \sqrt{n}}-\frac{(s-\alpha)}{2} n
$$

We have to prove

$$
\left|D G^{M}\right|>\lambda^{\left[c_{0} M / 2 \gamma p_{0}\right]+s}
$$

Now, $M=\left(\sum_{i=1}^{n} N_{i}\right)-1$,

$$
\frac{1-s+n \alpha}{2}>s+\frac{c_{0}}{2 \sqrt{p_{0}}}
$$


and $c_{0} / 2 s>4$ imply

$$
2 \sqrt{n}<\sqrt{c_{0} n / 2 s}<\sqrt{p_{0}}
$$

and (12.5a) follows from (12.5).

So i) is proved for the holes $\delta_{n}^{-M}=\left(F_{n-1} \circ h\right)^{-1} \delta_{n}^{-N}$. Any branch $f_{i_{n-1}} \circ \ldots \circ f_{i_{1}} \circ g$ is some composition of the form $f_{i_{n-1}} \circ \ldots \circ f_{i_{k}} \circ\left(f_{i_{k-1}} \circ \ldots \circ f_{i_{1}} \circ h\right)$, where $f_{i_{k-1}} \circ \ldots \circ f_{i_{1}} \circ h$ is a central branch of some previous step. Thus the same arguments prove i) for $\delta_{n}^{-M}=\left(F_{n-1} \circ g\right)^{-1} \delta_{n}^{-N}$ (the estimates are better in this case). If $\delta_{n}^{-M}=\hat{f}_{n}^{-1} \delta_{n}^{-N}, \mathrm{i}$ ) follows from i) and ii) of Step $n-1$.

Let $G^{-N} \delta_{n}=\delta_{n}^{-N} C[0,1] \backslash \delta_{n+1}$. Then we have $\max (\ell, n)=n+1$. Now i) follows for $G_{n+1}^{M}: \delta_{n+1}^{-M} \rightarrow \delta_{n+1}$ with $\delta_{n+1}^{-M} \subset[0,1] \backslash \delta_{n+1}$ because they are compositions of maps satisfying i) with $r \leqq n+1$. Similarly for $G_{n+1}^{M}: \delta_{n+1}^{-M} \rightarrow \delta_{n+1}, \delta_{n+1}^{-M} \subset[0,1] \backslash \Gamma_{\ell}$, $\ell>n+1$. This proves $i_{n+1}$.

Let $f_{n+11}=f_{i_{n}} \circ f_{i_{n-1}} \circ \ldots \circ f_{i_{1}} \circ g_{\lambda} \mid[0,1] \backslash \delta_{n+1}$. The induction conditions on $\left|D f_{i_{k}}\right|$ imply that $\left|D f_{n+11}\right|=\prod_{k=1}^{n}\left|D f_{i_{k}}\right| \cdot 2 \lambda\left|x-\frac{1}{2}\right|$ satisfies Lemma 7 . The same is true for $f_{n+11}=f_{i_{k}} \circ \hat{f}_{n}$, because of ii). Taking into account $i_{n+1}$, we obtain Lemma 7 for $f_{n+1 k}$ with $k>1$. Finally ii) at Step $n+1$ follows from i) and the assertion of Lemma 7 for $f_{n+1}$.

b) We shall use the following estimates for compositions of maps.

Let $g: B \rightarrow J$ be given by $g(x)=a x^{2}$, where $B=\left[x_{\min }, x_{\max }\right]$ and $J=\bigcup \Delta$, where int $\Delta_{1} \bigcap$ int $\Delta_{2}=\emptyset$ if $\Delta_{1} \neq \Delta_{2}$. Let $\Delta=\left[h_{\Delta}, h_{\Delta}+|\Delta|\right]$ and denote by $n(\Delta)$ the number of iterations corresponding to $\Delta$. Then $B=\bigcup g^{-1} \Delta$, where

and

$$
\left|g^{-1} \Delta\right|=\frac{1}{\sqrt{a}}\left(\sqrt{h_{\Delta}+|\Delta|}-\sqrt{h_{\Delta}}=\frac{|\Delta|}{\sqrt{a}\left(\sqrt{h_{\Delta}+|\Delta|}+\sqrt{h_{\Delta}}\right.}\right)
$$

$$
n\left(g^{-1} \Delta\right)=1+n(\Delta)
$$

Hence

$$
\begin{aligned}
\sum n\left(g^{-1} \Delta\right)\left|g^{-1} \Delta\right| & =\frac{1}{\sqrt{a}} \sum \frac{(1+n(\Delta))|\Delta|}{\sqrt{h_{\Delta}+|\Delta|}+\sqrt{h_{\Delta}}} \\
& =\frac{1}{\sqrt{a}} \sum \frac{|\Delta|}{\sqrt{h_{\Delta}+|\Delta|}+\sqrt{h_{\Delta}}}+\frac{1}{\sqrt{a}} \sum \frac{n(\Delta)|\Delta|}{\sqrt{h_{\Delta}+|\Delta|}+\sqrt{h_{\Delta}}} .
\end{aligned}
$$

Let us now consider $\left\{\Delta^{\prime}, f^{\prime}, n^{\prime}\right\}$, where int $\Delta_{1}^{\prime} \bigcap$ int $\Delta_{2}^{\prime}=\emptyset, n^{\prime}=n^{\prime}\left(\Delta^{\prime}\right)=n^{\prime}\left(f^{\prime}\right)$. Suppose every $f^{\prime}$ maps its domain onto the same interval, $f^{\prime}: \Delta^{\prime} \rightarrow J$, and $\mu\left(f^{\prime}, \Delta^{\prime}\right)$ $<c$. Let $\{\Delta, f, n\}$ be so that $\Delta C J$, int $\Delta_{1} \bigcap$ int $\Delta_{2}=\emptyset, n=n(\Delta)=n(f)$. Then

$$
\begin{aligned}
\sum_{\Delta, \Delta^{\prime}} n\left(f^{\prime-1}(\Delta)\right)\left|f^{\prime-1} \Delta\right| & <\left(\sum_{\Delta, \Delta^{\prime}}\left(n+n^{\prime}\right)|\Delta|\left|\Delta^{\prime}\right|\right) \frac{\exp (c)}{|J|} \\
& =\left[\left(\sum n|\Delta|\right)\left(\sum\left|\Delta^{\prime}\right|\right)+\left(\sum n^{\prime}\left|\Delta^{\prime}\right|\right)\left(\sum|\Delta|\right)\right] \frac{\exp (c)}{|J|}
\end{aligned}
$$

c) When estimating $\sum n(\Delta)|\Delta|$ after step $n$ of the induction construction we shall attribute to any preimage $\delta_{n}^{-N}$ mapped onto $\delta_{n}$ by $G_{n}^{N}$ the number of iterations $N$, 
ignoring the structure inside $\delta_{n}$. But when considering $\delta_{n}$ itself, we take into account this structure. This gives the estimate of $\sum n(\Delta)|\Delta|$ on any domain inside $\delta_{n}$. Then according to the construction of Sect. 3 we introduce at step $n+1$ the structure from $\delta_{n} \backslash \delta_{n+1}$ inside every domain $\left(\delta_{n} \backslash \delta_{n+1}\right)^{-N}$.

Before formulating the induction hypotheses of Proposition 3 we introduce a new notation. Let $\Delta_{0}=\Delta f \subset[0,1] \backslash \delta_{n}$ be a domain of some $f$, constructed after Step $n$. We define a "block" $B\left(\Delta_{0}\right)$ as a maximal interval containing $\Delta_{0}$, which doesn't contain any hole $\delta_{n}^{-k}$. If $B\left(\Delta_{0}\right) \bigcap \delta_{n}=\emptyset$, then $B\left(\Delta_{0}\right)=\bigcup \Delta_{i}$, where any $\Delta_{i}=\left[a_{i}, a_{i+1}\right]$, $i \in \mathbb{Z}$, is a domain of some $\Delta f_{\ell}, \ell \leqq n$. If $B\left(\Delta_{0}\right) \bigcap \delta_{n} \neq \emptyset$, then a part of the $\Delta_{i}$ are as above and the others are $\Delta\left(F_{n-1} \circ g\right)$ or $\Delta \hat{f}_{n}$.

After Step 2 we obtain two exceptional one-side blocks $B_{0}^{*}$, which contains 0 , and $B_{1}^{*}$, containing 1 , and for any $\tilde{B} \neq B_{0}^{*}, B_{1}^{*}, \tilde{B}=\tilde{B}_{1} \cup \tilde{B}_{2}$, where $\tilde{B}_{1}=g^{-n} B_{0}^{*}, \tilde{B}_{2}$ $=g^{-n} B_{1}^{*}$.

The structure of $B_{0}^{*}$ is : $B_{0}^{*}=\bigcup B_{0 i}, i=1,2, \ldots$, where $B_{0 i}=\bigcup \Delta_{i k}, k \in\left[1, n_{0}\right], n_{0}$ $=\operatorname{card}\left\{\Delta f_{1} \subset\left[0, \frac{1}{2}\right]\right\}, \Delta_{1 k}=\Delta f_{1}, \Delta_{i k}=\Delta f_{2 i-1}$ for $i \geqq 2$, and the corresponding number of iterations $N\left(\Delta_{i k}\right)=i$. The structure of $B_{1}^{*}$ is similar.

Let $\tilde{B}$ be some block of step $n+1$. Then either $\tilde{B}=\tilde{B}_{1} \bigcup \tilde{B}_{2}$, where $\tilde{B}_{1} \subset\left[a_{1}, a\right]$, $\tilde{B}_{2} \subset\left[a, a_{2}\right]$, and $\left[a_{1}, a\right],\left[a, a_{2}\right]$ are two adjacent intervals constructed at step $n$, $\tilde{B}_{1} \cap \tilde{B}_{2}=a$, and both $\tilde{B}_{1}, \tilde{B}_{2}$ are preimages of $B_{0}^{*}$ or $\tilde{B}_{1}^{*}$, or $\tilde{B}$ is some preimage of such blocks constructed at previous steps.

When constructing $\delta_{n+1}$ we shall take the precaution to choose two adjacent intervals $\Delta^{\prime} \subset[0,1] \backslash \delta_{n+1}$ and $\Delta^{\prime \prime} \subset \delta_{n+1}$ which are the preimages of $\Delta_{i k}$ with the same $i$. This can be done by moving if necessary the point $\xi_{1 n}$ of Sect. 10 a distance less than $\left(2 \lambda^{c_{1}}\right)^{-(n+1)}$ and still having (10.10) true.

Let $B_{+}\left(\Delta_{0}\right)=\bigcup\left(\Delta_{i} \subset B\left(\Delta_{0}\right), i>0\right), B_{-}\left(\Delta_{0}\right)=\bigcup\left(\Delta_{i} \subset B\left(\Delta_{0}\right), i<0\right)$. Then the preceding implies

$$
\min \left(\operatorname{mes} B_{+}\left(\Delta_{0}\right), \operatorname{mes} B_{-}\left(\Delta_{0}\right)\right)>\frac{1-\lambda^{-t}}{2}\left|\Delta_{0}\right| \text {. }
$$

(12.8) together with (4.6) imply the following

Property. Let $\Delta_{0}=\Delta f_{k} \subset B\left(\Delta_{0}\right) \subset \operatorname{Im} F_{n-1} \circ h(\lambda, x)$ be so that $F_{n-1} \circ h\left(\lambda, \frac{1}{2}\right) \notin B\left(\Delta_{0}\right)$. Then

$$
\mu\left(F_{n-1} \circ h(\lambda, x), \Delta_{0}\right)<3
$$

d) Let $\mathscr{D}_{n}=\Delta\left(F_{n-1} \circ h\right), F_{n-1}=f_{i_{n-1}} \circ \ldots \circ f_{i_{1}}$, and let $\Delta_{0}^{(n)}=\Delta f_{i_{n}}$ be so that $F_{n-1} \circ h\left(\lambda, \frac{1}{2}\right) \in \Delta_{0}^{(n)}$. Then $\mathscr{D}_{n+1}=\Delta\left(f_{i_{n}} \circ F_{n-1} \circ h\right)$. Let $B_{n}=B\left(\Delta_{0}^{(n)}\right)$ be the block of $\Delta_{0}^{(n)}$, $\mathscr{U}_{n}=\left(F_{n-1} \circ h\right)^{-1} B\left(\Delta_{0}^{(n)}\right)$. Notice that $\mathscr{D}_{n+1}$ may be equal to $\mathscr{D}_{n}$ (it is, if im $F_{n-1} \circ h(\lambda$, $x) \subset \Delta(0)$ - the first interval $\Delta f_{1}$ on $[0,1]$ (or $\subset \Delta(1)$ - the last one)), but always $\mathscr{U}_{n+1} \varsubsetneqq \mathscr{U}_{n}$.

We now formulate the induction hypotheses for the proof of Proposition 3. Let $R(n)=\max \left\{R: \mathscr{U}_{n-1} \subset \Gamma_{R}\right\}$ where $\Gamma_{R}=\left(\frac{1}{2}-\lambda^{-s R}, \frac{1}{2}+\lambda^{-s R}\right)$. Let $\sum_{n}^{k}=\sum N(\Delta)|\Delta|$ after step $n$, where $\Delta \subset \delta_{k} \backslash \delta_{k+1}$ if $k<n$, or $\Delta \subset \Gamma_{k} \backslash \Gamma_{k+1}$ if $k \geqq n$, are either intervals $\Delta f_{i}$, $i \leqq n, \Delta F_{n-1} \circ g, \Delta \hat{f}_{n}$ or holes $\delta_{n}^{-M} .\left(N\left(\delta_{n}^{-M}\right)=M\right.$ for holes.) Then for $k \leqq R(n)-1$,

i) $\sum_{n}^{k}<\frac{k^{3 / 2}}{\lambda^{s(1-\beta) k}} \sum_{i=0}^{n-1} \lambda^{-i t}$. 
Consider any $x_{0}, x_{1}, x_{2} \in \delta_{k-2} \backslash \delta_{k}$ (respectively $\Gamma_{k-2} \backslash \Gamma_{k}$ ), so that $x_{1} \in\left[x_{0}, x_{2}\right]$. Then for $k \leqq R(n)$

ii) $\sum_{\Delta C\left[x_{0}, x_{2}\right]} N(\Delta)|\Delta| /\left|x_{0}-x_{2}\right|<\lambda^{s \beta k}$

$$
\cdot\left(\sum_{\Delta \subset\left[x_{0}, x_{1}\right]} N(\Delta)|\Delta| /\left|x_{0}-x_{1}\right|\right)\left(\sum_{i=0}^{n-1} \lambda^{-i t}\right) .
$$

We have to prove (i) and (ii) for $n+1$ and $k \leqq R(n+1)-1$ (respectively $k \leqq R(n+1)$ ), where $R(n+1)=\max \left\{R \mid \mathscr{U}_{n} \subset \Gamma_{R}\right\}$.

We shall assume that the boundary points of $\delta_{n}, \delta_{n+1}, \mathscr{D}_{n}$ and $\mathscr{U}_{n}$ lie in $\left\{\lambda^{-s m}\right\}$, that is, $\delta_{k}=\left(\frac{1}{2}-\lambda^{-s k}, \frac{1}{2}+\lambda^{-s k}\right)$ for $k=n, n+1$, and for some $r, p \in \mathbb{Z}$,

$$
\begin{aligned}
& \mathscr{D}_{n}=\left(\frac{1}{2}-\lambda^{-s r}, \frac{1}{2}+\lambda^{-s r}\right) \\
& \mathscr{U}_{n}=\left(\frac{1}{2}-\lambda^{-s(r+p)}, \frac{1}{2}+\lambda^{-s(r+p)}\right) .
\end{aligned}
$$

In addition we suppose $\frac{c_{0}}{2 s}$ and $\frac{\alpha}{s} n$ to be integers. The reader can check there is no loss of generality here.

Let $F_{n-1} \circ h\left(\lambda, \frac{1}{2}\right) \in \delta_{q-1} \backslash \delta_{q}$. According to the main construction $q \leqq\left(1-\frac{\alpha}{s}\right) n$. Let $N\left(f_{i_{k}}\right)=N_{k}, k \in[1, n-1]$. Lemma 7 implies

$$
\left|D f_{i_{k}}\right|>\lambda^{\frac{N_{k} c_{0}}{2 \sqrt{k}}+s}
$$

As $\frac{1}{2}-\lambda^{-s r}$ is a root of the equation

$$
F_{n-1} \circ[\lambda x(1-x)]=F_{n-1} \circ h\left(\lambda, \frac{1}{2}\right) \quad(\bmod 1)
$$

we have

$$
\frac{1}{\lambda^{s r}}<\frac{1}{\left[\lambda \prod_{k=1}^{n-1}\left|D f_{i k}\right|\right]^{1 / 2}}<\exp \left[-\frac{1}{2}\left(1+\sum_{k=1}^{n-1}\left(\frac{N_{k} c_{0}}{2 \sqrt{k}}+s\right)\right) \ell n \lambda\right]
$$

Hence

$$
\begin{aligned}
& s r>\frac{1}{2}\left[1+(n-1) s+\frac{c_{0}}{2} \sum_{k=1}^{n-1} N_{k} / \sqrt{k}\right] \\
& >\frac{1}{2}\left[1+(n-1) s+\frac{c_{0}}{2 \sqrt{n-1}} \sum_{k=1}^{n-1} N_{k}\right] .
\end{aligned}
$$

This implies that the number of iterations $N\left(F_{n-1} \circ h\right)=1+\sum_{k=1}^{n-1} N_{k}$ satisfies

$$
N\left(F_{n-1} \circ h\right)<\frac{4 s}{c_{0}} r \sqrt{n-1}-\frac{2 s}{c_{0}}(n-1)^{3 / 2} .
$$

Taking into account that $r>c_{0} n / 2 s$, we obtain

$$
N\left(F_{n-1} \circ h\right)<2\left(\frac{2 s r}{c_{0}}\right)^{3 / 2}-\frac{2 s}{c_{0}}(n-1)^{3 / 2} .
$$


We shall denote $\Delta_{0}^{(n)}$ by $\Delta_{0}$ and $B_{n}$ by $B$ below. As $F_{n-1} \circ h\left(\lambda, \frac{1}{2}\right) \in \Delta_{0}$, $\operatorname{im} F_{n-1} \circ h(\lambda, x)$ contains either $B_{+}\left(\Delta_{0}\right)$, or $B_{-}\left(\Delta_{0}\right)$. Suppose the former. The number of iterations $N\left(\Delta_{i}\right)$ are either increasing, or they decrease till some $N_{\text {min }}$, and then increase up to infinity. Let $\mathscr{S}=\mathrm{im} F_{n-1} \circ h(\lambda, x) \bigcap B$. The properties of blocks are so that in the second case $|\mathscr{P}|=2 n_{0} \cdot\left|\Delta_{\min }\right|(1+\varepsilon)$, where $\Delta_{\text {min }}$ is any interval corresponding to $N_{\min }, n_{0}=\operatorname{card}\left\{\Delta f_{1} \subseteq\left[0, \frac{1}{2}\right] \backslash \delta_{1}\right\}<\lambda, \varepsilon<\lambda^{-t}$. In the first case more than $1-\varepsilon$ of $|\mathscr{S}|$ consists of intervals with $N\left(\Delta_{i}\right)=N\left(\Delta_{0}\right)$, and $N\left(\Delta_{i}\right)$ $=N\left(\Delta_{0}\right)+1$ (the distribution depends on the number of first $\Delta_{i}$ with $N\left(\Delta_{i}\right)$ $\left.=N\left(\Delta_{0}\right)\right)$. In both cases we have

$$
\sum_{\Delta \subset \mathscr{S}} N(\Delta)|\Delta|<(1+\varepsilon) \cdot N(\mathscr{S}) \cdot|\mathscr{S}|
$$

and

$$
|\mathscr{P}|<2 \cdot \lambda \cdot(1+\varepsilon)\left|D f_{k}^{-(N(\mathscr{S})-1)}\right|
$$

where $N(\mathscr{S})=N_{\min }$ in the second case, $N(\mathscr{S})=N\left(\Delta_{0}\right)+1$ in the first case. Taking into account that $\Delta_{0}, \Delta_{\min } \cong B \subseteq[0,1] \backslash \delta\left(1-\frac{\alpha}{s}\right) n$ and thus $f_{k}^{N_{\min }}: \Delta_{\min } \rightarrow[0,1]$ (correspondingly $f_{k}^{N\left(\Delta_{0}\right)}$ ) satisfies Lemma 7 with $\left(1-\frac{\alpha}{\mathrm{s}}\right) n$, and proceeding as above when deriving (12.10), we obtain for $\mathscr{U}_{n}=\left(F_{n-1} \circ h(\lambda, x)\right)^{-1} \mathscr{S}=\Gamma_{r+p}$

$$
N\left(F_{n-1} \circ h\right)+N(\mathscr{S})<2\left(\frac{2 s}{c_{0}}(r+p)\right)^{3 / 2}-\frac{2 s}{c_{0}}(n-1)^{3 / 2} .
$$

e) As $\Gamma_{r}=\mathscr{D}_{n}=\Delta\left(F_{n-1} \circ h\right)$ consists of a unique $\Delta$, after step $n$ we have $\sum_{n}^{k}$ $=N\left(F_{n-1} \circ h\right) \cdot 2\left(\lambda^{-s k}-\lambda^{-s(k+1)}\right)$ for $k \geqq r$.

Let us estimate $\sum n(\Delta)|\Delta|$ after taking the first compositions $f_{i} \circ F_{n-1} \circ h$ on every $\Gamma_{k} \backslash \Gamma_{k+1}{ }^{\text {def }} \tilde{\Gamma}_{k}, r \leqq k<r+p$. We shall denote this sum by $\sum_{n+11}^{k}$.

Let $\mathscr{S}_{0}=\mathscr{S}$, and let $\mathscr{S}_{i}$ be the $\lambda^{2 s i}$-enlargement of $\mathscr{S}_{0}$ with center $F_{n-1} \circ h\left(\lambda, \frac{1}{2}\right)$. Then $\Gamma_{r+p}=\left(F_{n-1} \circ h\right)^{-1} \mathscr{S}_{0}, \tilde{\Gamma}_{r+p-i}=\left(F_{n-1} \circ h\right)^{-1}\left(\mathscr{S}_{i} \mid \mathscr{S}_{i-1}\right), i=1,2, \ldots, p$. Applying (12.7) to $\left\{\Delta \subseteq \mathscr{S}_{i} \mid \mathscr{S}_{i-1}\right\}$ and $\Delta^{\prime}=\Delta \mathrm{F}_{n-1}$ we obtain using $b_{2 n}$

$$
\begin{aligned}
& \left.\sum_{\Delta C \mathscr{S}_{i} \backslash \mathscr{S}_{i-1}}\left(N\left(F_{n-1}\right)+n(\Delta)\right)\left|F_{n-1}^{-1} \Delta\right|<\left(1+O\left(\lambda^{-\gamma}\right)\right) \mid \Delta F_{n-1}\right) \mid \\
& \left(N\left(F_{n-1}\right)\left|\mathscr{S}_{i} \backslash \mathscr{S}_{i-1}\right|+\sum_{\Delta \subset \mathscr{S}_{i} \backslash \mathscr{S}_{i-1}} n(\Delta)|\Delta|\right) .
\end{aligned}
$$

Applying (12.6) to $\left.F_{n-1}^{-1}\left(\mathscr{S}_{i}\right) \mathscr{S}_{i-1}\right)$ we have $h_{\Delta}>\lambda^{1-2 s(r+p-(i-1))}$ and consequently

$$
\begin{aligned}
\sum_{n+11}^{r+p-i}= & \sum_{\left(F_{n-1}{ }^{\circ}\right)^{-1} \Delta}\left(N\left(F_{n-1}\right)+n(\Delta)+1\right)\left|\left(F_{n-1} \circ h\right)^{-1} \Delta\right| \\
< & 2^{-1} \lambda^{-1+s(r+p-(i-1))}\left(1+O\left(\lambda^{-\gamma}\right)\right) \Delta F_{n-1} \\
& \cdot\left(\left|\mathscr{S}_{i}\right| \mathscr{S}_{i-1}\left|+N\left(F_{n-1}\right)\right| \mathscr{S}_{i}\left|\mathscr{S}_{i-1}\right|+\sum_{\Delta \subset \mathscr{S}_{i} \backslash \mathscr{S}_{2-1}} n(\Delta)|\Delta|\right) .
\end{aligned}
$$

We shall assume $\operatorname{im} F_{n-1} \circ h(\lambda, x) \cong\left[0, \frac{1}{2}\right]$, and leave to the reader the modifications corresponding to another position of $\operatorname{im} F_{n-1} \circ h(\lambda, x)$ in $[0,1]$. Let 
$\ell=\max \left\{i: \mathscr{S}_{i} \leqq \delta_{q-2} \backslash \delta_{q}\right\}$. Then for $i \leqq \ell$ we can apply (ii). Together with (12.11) this gives

$$
\begin{aligned}
\sum_{\Delta C \mathscr{S}_{i} \backslash \mathscr{S}_{2-1}} n(\Delta)|\Delta| & <\sum_{\Delta \subset \mathscr{S}_{i}} n(\Delta)|\Delta|<\lambda^{s \beta\left(1-\frac{\alpha}{s}\right) n} \cdot N\left(\mathscr{S}_{0}\right) \cdot\left|\mathscr{S}_{0}\right| \cdot \lambda^{2 s i} \cdot(1+\varepsilon) \\
& =N\left(\mathscr{S}_{0}\right) \cdot\left|\mathscr{S}_{i}\right| \mathscr{S}_{i-1} \mid \cdot\left(1-\lambda^{-2 s}\right)^{-1} \cdot(1+\varepsilon) \cdot \lambda^{\beta(s-\alpha) n} .
\end{aligned}
$$

Substituting this estimate in (12.15), we obtain

$$
\sum_{n+1}^{r+p-1}<\frac{\left(1+O\left(\lambda^{-\gamma}\right)\right) \cdot\left|\Delta F_{n-1}\right| \cdot\left|\mathscr{S}_{i}\right| \mathscr{S}_{i-1} \mid\left(1+N\left(F_{n-1}\right)+N\left(\mathscr{S}_{0}\right)\left(1+\varepsilon_{1}\right) \cdot \lambda^{\beta(s-\alpha) n}\right)}{2 \lambda^{1-s(r+p-(i-1))}} .
$$

We have by definition $\mathscr{S}_{k} \mid \mathscr{S}_{k-1}=F_{n-1} \circ h\left(\tilde{\Gamma}_{r+p-k}\right)$ and using $b_{2 n}$, this implies

$$
\left|\Delta F_{n-1}\right| \cdot\left|\mathscr{S}_{k}\right| \mathscr{P}_{k-1} \mid=\lambda^{1-2 s(r+p-k)} \cdot\left(1+O\left(\lambda^{-\gamma}\right)\right)\left(1-\lambda^{-2 s}\right) \text {. }
$$

Thus

$$
\sum_{n+1}^{r+p-i}<\frac{\left(1+\varepsilon_{2}\right) \cdot \lambda^{s} \cdot\left(1+N\left(F_{n-1}\right)+N\left(\mathscr{S}_{0}\right) \lambda^{\beta(s-\alpha) n}\right)}{2 \lambda^{s(r+p-i)}} .
$$

Consequently, by (12.13) this implies

$$
\begin{gathered}
\sum_{n+1}^{r+p-i}<\frac{\left(1+\varepsilon_{12.17}\right) \cdot \lambda^{s} \cdot\left(\frac{2 s}{c_{0}}(r+p)\right)^{3 / 2} \lambda^{\beta(s-\alpha) n}}{\lambda^{s(r+p-i)}} \\
<\left(1+\varepsilon_{12.17}\right)\left(\frac{2 s}{c_{0}}\right)^{3 / 2} \frac{(r+p-i)^{3 / 2}}{\lambda^{s(1-\beta)(r+p-i)}} \cdot \frac{\lambda^{s \beta\left(1-\frac{\alpha}{s}\right) n+s+2 \beta}}{\lambda^{s \beta(r+p-i)}} .
\end{gathered}
$$

Thus for $1 \leqq i \leqq \ell$ we have on $\tilde{\Gamma}_{r+p-i}$ the analogue of assumption (i) but with an additional factor less than (we use $\left.n<\frac{2 s}{c_{0}} r\right)$

$$
\frac{\lambda^{s+2 \beta}}{\lambda^{s \beta\left(r+p-i-\left(1-\frac{\alpha}{s}\right) n\right)}}<\frac{\lambda^{s+2 \beta}}{\left[\lambda^{\left.s \beta p-i+r\left(1-\frac{2(s-\alpha)}{c_{0}}\right)\right]} .\right.}
$$

In a general case we have $\ell<p$ (this is not so only if $F_{n-1} \circ h\left(\lambda, \frac{1}{2}\right) \in[0,1] \backslash \delta_{3}$ ), and we have also to estimate $\sum_{n+11}^{r+j}, 0 \leqq j<p-\ell$. Let us consider

$$
\mathscr{S}_{\ell+1}=\lambda^{2 s(\ell+1)} \mathscr{S}_{0}, \ldots, \mathscr{S}_{p}=\lambda^{2 s p} \cdot \mathscr{S}_{0}=\operatorname{im} F_{n-1} \circ h(\lambda, x) \text {. }
$$

We have $\mathscr{S}_{p} \backslash \mathscr{P}_{p-1}=\left[0, a_{p-1}\right]$, where $\frac{1}{2}-\lambda^{-2 s} / 2 \approx a_{p-1} \in \delta_{2} \backslash \delta_{3}$,

$$
\begin{gathered}
\mathscr{S}_{p-1} \backslash \mathscr{S}_{p-2}=\left[a_{p-1}, a_{p-2}\right], \quad \frac{1}{2}-\frac{1}{2 \lambda^{4 s}} \approx a_{p-2} \in \delta_{4} \backslash \delta_{5} \ldots, \\
\mathscr{S}_{\ell+1} \backslash \mathscr{S}_{\ell}=\left[a_{\ell+1}, a_{\ell}\right], \\
\left.\frac{1}{2}-\frac{1}{2} \lambda^{-2 s(p-\ell-1}\right) \approx a_{\ell+1} \in \delta_{2(p-\ell-1)} \backslash \delta_{2(p-\ell-1)+1}, \\
\frac{1}{2}-\frac{1}{2} \lambda^{-2 s(p-\ell)} \approx a_{\ell} \in \delta_{2(p-\ell)} \backslash \delta_{2(p-\ell)+1} .
\end{gathered}
$$


By construction $\mathscr{S}_{\ell}=\left[a_{\ell}, F_{n-1} \circ h\left(\lambda, \frac{1}{2}\right)\right]$ is the last enlargement of $\mathscr{S}_{0}$ which lies in $\delta_{q-2} \backslash \delta_{q}$. Hence either $a_{\ell} \in \delta_{q-1} \backslash \delta_{q}$, or $a_{\ell} \in \delta_{q-2} \backslash \delta_{q-1}$. For definiteness let $a_{\ell} \in \delta_{q-1} \backslash \delta_{q}$. Then $q=2(p-\ell)+1, \ell=p-\frac{q-1}{2}$,

$$
\mathscr{S}_{\ell+j} \mid \mathscr{S}_{\ell+j-1} \subseteq \delta_{2(p-\ell-j)} \backslash \delta_{2(p-\ell-j)+3}, \quad j \in[1, p-\ell],
$$

where $\delta_{0}=\left[0, \frac{1}{2}\right]$.

(i) for $\delta_{k}$ with $k=2(p-\ell-j), k+3 \leqq q \leqq\left(1-\frac{\alpha}{s}\right) n$ implies

$$
\sum_{n}^{k}+\sum_{n}^{k+1}+\sum_{n}^{k+2}<\frac{k^{3 / 2}}{\lambda^{s(1-\beta) k}}\left(1+\varepsilon_{3}\right) \text {. }
$$

By construction

$$
\frac{1-\varepsilon_{4}}{2} \lambda^{-2 s(p-\ell-j)}<\left|\mathscr{P}_{\ell+j}\right| \mathscr{S}_{\ell+j-1} \mid<\lambda^{-2 s(p-\ell-j)} .
$$

Hence using (12.7) we obtain similarly to (12.14) for $j \in[1, p-\ell]$

$$
\begin{aligned}
& \sum_{\Lambda \subset \mathscr{S}_{\ell+j} \backslash \mathscr{S}_{\ell+j-1}}\left(N\left(F_{n-1}\right)+n(\Delta)\right)\left|F_{n-1}^{-1}(\Delta)\right| \\
< & \left(1+O\left(\lambda^{-\gamma}\right)\right)\left|\Delta F_{n-1}\right|\left\{N\left(F_{n-2}\right)\left|\mathscr{S}_{\ell+j}\right| \mathscr{S}_{\ell+j-1} \mid\right. \\
& \left.+[2(p-\ell-j)]^{3 / 2} \lambda^{-s(1-\beta) 2(p-\ell-j)}\right\} \\
< & \left(1+\varepsilon_{12.19}\right)\left|\Delta F_{n-1}\right|\left|\mathscr{S}_{\ell+j}\right| \mathscr{S}_{\ell+j-1} \mid \\
& \cdot\left[N\left(F_{n-1}\right)+2(2(p-\ell-j))^{3 / 2} \lambda^{2 s \beta(p-\ell-j)}\right] .
\end{aligned}
$$

By construction

$$
\lambda^{2 s} \cdot \operatorname{dist}\left(a_{\ell}, F_{n-1} \circ h\left(\lambda, \frac{1}{2}\right)\right)>\frac{1-\lambda^{-s}}{\lambda^{s(q-2)}} .
$$

This implies

$$
h_{\Delta}>\left(1-\lambda^{-s}\right)\left|\Delta F_{n-1}\right| \lambda^{-s(2(p-\ell)+1)}
$$

on $F_{n-1}^{-1}\left(\mathscr{S}_{\ell+1} \backslash \mathscr{S}_{\ell}\right)$ and

$$
h_{\Delta}>\frac{\left(1-\lambda^{-s}\right)\left|\Delta F_{n-1}\right|}{\lambda^{s(2(p-\ell-j)+3)}}
$$

on $\left.F_{n-1}^{-1}\left(\mathscr{S}_{\ell+j}\right) \mathscr{S}_{\ell+j-1}\right)$. Applying (12.6) we obtain from (12.19) that

$$
\begin{aligned}
\sum_{n+1}^{r+p-(\ell+j)}<\left(1+\varepsilon_{12.20}\right)\left|\Delta F_{n-1}\right|^{1 / 2}\left|\mathscr{S}_{\ell+j}\right| \mathscr{S}_{\ell+j-1} \mid\left[N\left(F_{n-1}\right)+1\right. \\
\left.+(2(p-\ell-j))^{3 / 2} 2 \lambda^{2 s \beta(p-\ell-j)}\right] \lambda^{-1 / 2} \lambda^{-s(p-\ell-j+3 / 2)}
\end{aligned}
$$

Now, (12.16) with $k=\ell+j$ implies

$$
\left|\Delta F_{n-1}\right|\left|\mathscr{S}_{\ell+j}\right| \mathscr{S}_{\ell+j-1} \mid<\frac{\sqrt{\lambda} \lambda^{-s(p-\ell-j)}\left(1+\varepsilon_{5}\right)}{\lambda^{s(r+p-\ell-j)}} .
$$


Substituting this into (12.20) we obtain

$$
\sum_{n+1}^{r+p-(\ell+j)}<\left(1+\varepsilon_{12.21}\right) \lambda^{3 s / 2} \frac{N\left(F_{n-1}\right)+1+(2(p-\ell-j))^{3 / 2} \lambda^{2 s \beta(p-\ell-j)}}{\lambda^{s(r+p-\ell-j)}} .
$$

According to $(12.10), N\left(F_{n-1}\right)+1<2\left(2 s r / c_{0}\right)^{3 / 2}$. Because

$$
2(p-\ell)<\left(1-\frac{\alpha}{s}\right) n<\left(1-\frac{\alpha}{s}\right) \frac{2 s r}{c_{0}}
$$

we can rewrite $(12.21)$ as

$$
\begin{gathered}
\sum_{n+11}^{r+p-\ell-j}<\left(1+\varepsilon_{12.22}\right)\left(\frac{2 s}{c_{0}}\right)^{3 / 2} \frac{r^{3 / 2}}{\lambda^{s(1-\beta)(r+p-\ell-j)}} \\
\cdot \frac{2 \lambda^{\frac{3 s}{2}}}{\lambda^{s \beta(r+p-\ell-j)} \overline{\left(1-\frac{2 s}{c_{0}}\right)}} \cdot
\end{gathered}
$$

Thus for $0 \leqq k<p-\ell$ we have on $\tilde{\Gamma}_{r+k}$ an additional exponentially small factor compared with the assumption (i), as well as for $p-\ell \leqq k<p$ (see (12.18)).

f) In order to estimate the contribution of terms in $\left(\delta_{n}-\delta_{n+1}\right)^{-M}$ we first do it in $\delta_{n} \backslash \delta_{n+1}$.

Step $n+1$ on $\delta_{n} \backslash \delta_{n+1}$ divides into subspteps $\ell=1,2, \ldots$ corresponding to the construction of $f_{n+1 \ell}$ (see Sect. 3).

We use the following notation: $\Delta$ is any interval $\Delta F_{n-1} \circ g, \Delta \hat{f}_{n}, \Delta f_{n+1 \ell} ; \delta$ is any hole $\delta_{n}^{-M}, \delta_{n+1}^{-N} \subseteq \delta_{n} \backslash \delta_{n+1} ; n(\Delta), n(\delta)$ are corresponding numbers of iterations. $n, \ell$

Let $i_{n \ell}=\sum|\Delta|$ after substep $\ell$ of step $n+1$ and with the same meaning of indices

$$
h_{n \ell}=\sum|\delta| ; \quad x_{n \ell}=\sum n(\Delta)|\Delta| ; \quad y_{n \ell}=\sum n(\delta)|\delta| .
$$

We consider also the corresponding sums on $[0,1] \backslash \delta_{n}$ namely

$$
I_{n}=\sum|\Delta|, \quad \Delta \subseteq[0,1] \backslash \delta_{n},
$$

after step $n$ :

$$
H_{n}=\sum|\delta| ; \quad X_{n}=\sum n(\Delta)|\Delta| ; \quad Y_{n}=\sum n(\delta)|\delta| .
$$

Then $I_{n}+H_{n}+\left|\delta_{n}\right|=1, i_{n \ell}+h_{n \ell}+\left|\delta_{n+1}\right|=\left|\delta_{n}\right|$ for all $\ell$. Besides, let $\ell=0$ correspond to $i, h, x, y$ constructed after step $n$, and $\ell=\infty$ after step $n+1$, so that $(n, \infty)$ equals $(n+1,0)$.

We may assume all the compositions to be linear (see Remark XII/1 below) and thus using (12.7) we obtain

$$
\left.\begin{array}{rl}
i_{n 1} & =i_{n 0}\left(1-H_{n}-\left|\delta_{n}\right|\right) \\
h_{n 1} & =h_{n 0}+i_{n 0}\left(H_{n}+\left|\delta_{n}\right|\right) \\
x_{n 1} & =x_{n 0} I_{n}+X_{n} \cdot i_{n 0} \\
y_{n 1} & =y_{n 0}+x_{n 0}\left(H_{n}+\left|\delta_{n}\right|\right)+Y_{n} \cdot i_{n 0} \cdot
\end{array}\right\}
$$


The holes and intervals of subsequent substeps $1=2,3, \ldots$ are obtained using compositions of maps $\tilde{G}_{n}: \delta_{n}^{-M} \rightarrow \delta_{n}$, so that after substep $\ell$ the remaining preimages of $\delta_{n}$ are of the form $\tilde{G}_{n_{\ell}}^{-1} \ldots \ldots \tilde{G}_{n_{1}}^{-1} \delta_{n}$ and preimages of $\delta_{n+1}$ are $\tilde{G}_{n_{\ell}}^{-1} \circ \ldots \circ \tilde{G}_{n_{1}}^{-1} \delta_{n+1}, i=1,2, \ldots, \ell-1$ (compare with (3.6), (3.7)). Let $\tilde{h}_{n \ell}$ and $\tilde{y}_{n \ell}$ correspond to preimages of $\delta_{n}$ and $\tilde{h}_{n \ell}, \tilde{y}_{n \ell}$ to preimages of $\delta_{n+1}$. With this notation we have

$$
\tilde{\tilde{y}}_{n 1}=\tilde{\tilde{h}}_{n 1}=0, \quad h_{n \ell}=\tilde{h}_{n \ell}+\tilde{\tilde{h}}_{n \ell}, \quad y_{n \ell}=\tilde{y}_{n \ell}+\tilde{\tilde{y}}_{n \ell},
$$

and for $\ell \geqq 2$

$$
\begin{aligned}
& i_{n \ell}=i_{n \ell-1}+i_{n \ell-1} \cdot \tilde{h}_{n \ell-1} \cdot\left|\delta_{n}\right|^{-1} \\
& \tilde{h}_{n \ell}=\tilde{h}_{n \ell-1} \cdot \tilde{h}_{n \ell-1} \cdot\left|\delta_{n}\right|^{-1} ; \\
& \tilde{h}_{n \ell}=\tilde{h}_{n \ell-1}+\tilde{h}_{n \ell-1} \cdot \frac{\tilde{h}_{n \ell-1}+\left|\delta_{n+1}\right|}{\left|\delta_{n}\right|} \\
& x_{n \ell}=x_{n \ell-1}+\left(\tilde{y}_{n \ell-1} \cdot i_{n \ell-1}+x_{n \ell-1} \cdot \tilde{h}_{n \ell-1}\right) \cdot\left|\delta_{n}\right|^{-1} \\
& \tilde{y}_{n \ell}=2 \tilde{y}_{n \ell-1} \cdot \tilde{h}_{n \ell-1} \cdot\left|\delta_{n}\right|^{-1} ; \\
& \tilde{y}_{n \ell}=\tilde{y}_{n \ell-1}+\frac{\tilde{y}_{n \ell-1} \cdot \tilde{h}_{n \ell-1}+\tilde{y}_{n \ell-1} \cdot \tilde{h}_{n \ell-1}+\tilde{y}_{n \ell-1}\left|\delta_{n+1}\right|}{\left|\delta_{n}\right|} .
\end{aligned}
$$

According to Sect. 10, to any hole $\delta_{n}^{-M}=G_{n}^{-M}\left(\delta_{n}\right)$ there corresponds uniquely a set $L_{n}^{-M}=G_{n}^{-M}(L)$. As for any interval $\Delta \leqq L_{n}^{-M}, n(\Delta)>n\left(\delta_{n}^{-M}\right)$; this implies

$$
\tilde{h}_{n 1}<\left(\left|\delta_{n}\right|-\left|\delta_{n+1}\right|\right)(1+\varepsilon) \cdot \lambda^{-2 \alpha n} .
$$

Using $\frac{\left|\delta_{n+1}\right|}{\left|\delta_{n}\right|}<\left(1+o\left(\lambda^{-s(n+1)}\right) \lambda^{-s}\right.$, the recurrent formulas (12.24) give

$$
\begin{aligned}
& x_{n \infty}=x_{n+10}<\left(x_{n 1}+y_{n 1}\right)\left(1+O\left(\lambda^{-2 \alpha n}\right)\right) ; \\
& y_{n \infty}=y_{n+10}<y_{n 0} \cdot \frac{1+o\left(\lambda^{-t n}\right)}{\lambda^{s}} .
\end{aligned}
$$

The induction hypotheses imply

$$
\begin{aligned}
X_{n} & <1-\frac{2}{\lambda^{s}}+\left(\sum_{k=1}^{n} \frac{k^{3 / 2}}{\lambda^{s(1-\beta) k}}\right)\left(\sum_{i=0}^{n-1} \lambda^{-t i}\right)<1+\varepsilon_{1} \\
x_{n 0} & <\frac{n^{3 / 2}}{\lambda^{s(1-\beta) n}} \sum_{i=0}^{n-1} \lambda^{-t i} \\
i_{n 0} & <2\left(1+o\left(\lambda^{-s n}\right)\right) \cdot\left(1-\lambda^{-s}\right) \cdot \lambda^{-s n} \\
I_{n} & <1-2 \lambda^{-s n} \\
H_{n}+\left|\delta_{n}\right| & <\left[\lambda^{s} \cdot\left(1+\varepsilon_{2}\right)\right]^{-n}
\end{aligned}
$$

By the above reasons

$$
y_{n 0}<x_{n 0} \cdot \lambda^{-2 \alpha n} \cdot(1+\varepsilon) ; \quad Y_{n}<X_{n} \cdot \lambda^{-2 \alpha n} \cdot(1+\varepsilon) .
$$


Using (12.23) we obtain

$$
\sum_{n+1}^{n}=x_{n+10}+y_{n+10}<\left(x_{n 0}+\frac{2}{\lambda^{s n}}\right)\left(1+O\left(\lambda^{-2 \alpha n}\right)\right)<\frac{n^{3 / 2}}{\lambda^{s(1-\beta) n}} \sum_{i=0}^{n} \lambda^{-t i}
$$

which proves $i_{n+1}$ for $\delta_{n} \backslash \delta_{n+1}$. The proof is similar for $\delta_{k} \backslash \delta_{k+1}, 1 \leqq k<n$.

Now we can estimate the contribution of $\sum n(\Delta)|\Delta|$ in every hole $\left(\delta_{n} \backslash \delta_{n+1}\right)^{-M}$ on $\tilde{\Gamma}_{r+i}=\Gamma_{r+i} \backslash \Gamma_{r+i+1}, i \in[0, p-1]$.

Though we cannot correspond the $\lambda^{2 \alpha n}$-enlargement to any $\delta_{n}^{-M}$, we can consider its $\lambda^{\alpha n / 2}$-enlargement. The construction of Sect. 10 gives, as above,

$$
\sum_{\delta \subset \tilde{\Gamma}_{r+1}}|\delta|<\frac{\left|\tilde{\Gamma}_{r+i}\right|(1+\varepsilon)}{\lambda^{\alpha n / 2}} ; \quad \sum_{\delta \subset \Gamma_{r+i}} n(\delta)|\delta|<\left(\sum_{\Delta \subset \tilde{\Gamma}_{r+i}} n(\Delta)|\Delta|\right) \frac{1+\varepsilon}{\lambda^{\alpha n / 2}} .
$$

Using (12.7) with $\mu=O\left(\lambda^{-\alpha n / 2}\right)$ we obtain after step $(n+1)$

$$
\begin{aligned}
\sum_{n+1}^{r+i} & <\sum_{n+11}^{r+i}+\left(\sum_{n+1}^{r+i} \cdot\left(1-\lambda^{-s}\right)\left|\delta_{n}\right| \cdot \lambda^{-\alpha n / 2}+\sum_{n+1}^{n} \cdot\left|\tilde{\Gamma}_{r+i}\right| \cdot \lambda^{-\alpha n / 2}\right) \frac{1+O\left(\lambda^{-\alpha n / 2}\right)}{\left|\delta_{n}\right|} \\
& \left.<\left(1+\varepsilon_{12.26}\right) \sum_{n+11}^{r+i}+\frac{n^{3 / 2}}{\lambda^{s(r+i)}} \cdot \frac{\lambda^{s \beta n}}{\lambda^{\alpha n / 2}}\right) .
\end{aligned}
$$

Thus we still have for $\sum_{n+1}^{r+i}$ an exponentially better estimate than that required by (i).

This proves $\left(i_{n+1}\right)$ for $k \in[r, R(n+1)-1]$. Now $\tilde{\Gamma}_{k} k \in[R(n), r-1]$ are contained in the union of preimages $\left(F_{n-2} \circ h\right)^{-1} \Delta_{i}^{(n-1)}$, where $\Delta_{i}^{(n-1)} C B_{n-1}\left(\Delta_{0}^{(n-1)}\right)$. One obtains $\left(i_{n+1}\right)$ for such $\tilde{\Gamma}_{k}$ in a similar way, using the construction of block $B_{n-1}$ (the estimates are better in this case).

In order to obtain $\left(i_{n+1}\right)$ for $n+1 \leqq k<R(n)-1$, we notice that at step $m(k)$ corresponding to the first consideration of $\Gamma_{k} \backslash \Gamma_{k+1}$, we have on $\Gamma_{k} \backslash \Gamma_{k+1}$ an exponential reserve by comparison with $\left(i_{m(k)}\right)$. (12.7) and Property (12.9) imply that the nonlinearity at $\operatorname{Step}(m(k)+1)$ gives an additional factor less than 3 . Any of the subsequent steps implies the diminishing of the maximal interval $\Delta \subset \Gamma_{k} \backslash \Gamma_{k+1}$ at least $3 \lambda^{c_{0}}$ times (because of taking compositions), and we obtain the following:

Remark XII/1. The total non-linear effect of steps $m(k)+1, m(k)+2, \ldots$ on $\Gamma_{k} \backslash \Gamma_{k+1}$ is less than

$$
\exp \left(3 \cdot \sum_{n \subset 0}^{\infty} \lambda^{-c_{0} n}\right)
$$

In particular this shows that when proving (i) for $\Gamma_{k} \backslash \Gamma_{k+1}$ it suffices to consider only step $m(k)$.

g) In order to prove $\left(i i_{n+1}\right)$ of Proposition 3 we consider three points $x_{0}, x_{1}$, $x_{2} \in \Gamma_{r+i-2} \backslash \Gamma_{r+i}$ and their images under $F_{n-1} \circ h(\lambda, x)$. We may suppose $x_{2}$ to be closer to $\frac{1}{2}$ than $x_{0}$, (otherwise $h_{\Delta}$ for $\Delta \subset\left[x_{1}, x_{2}\right]$ is larger than for $\Delta C\left[x_{0}, x_{1}\right]$ and an estimate for $x_{0}, x_{1}, x_{2}$ is better than for their images).

Let $Q_{1}=F_{n-1} \circ h\left[x_{0}, x_{1}\right], Q_{2}=F_{n-1} \circ h\left[x_{0}, x_{2}\right]$. Using (12.7) and (12.6) with $h_{\Delta}$ $\geqq h\left(x_{2}\right) \geqq \lambda^{1-2 s(r+i)}$ we obtain

$$
\sum_{\Delta \subset\left[x_{0}, x_{2}\right]} n(\Delta)|\Delta|<\frac{\left.1+O\left(\lambda^{-\gamma}\right)\right)\left|\Delta F_{n-1}\right|\left(\left(N\left(F_{n-1}\right)+1\right)\left|Q_{2}\right|+\sum_{\Delta \subset Q_{2}} n(\Delta)|\Delta|\right)}{2 \sqrt{\lambda} \lambda^{-s(r+i)}} .
$$


For $\Delta \subset\left[x_{0}, x_{1}\right] h_{\Delta} \leqq h\left(x_{0}\right) \leqq \lambda^{1-2 s(r+i)+4 s}$. Hence

$\sum_{\Delta \subset\left[x_{0}, x_{1}\right]} n(\Delta)|\Delta|>\frac{\left(1-O\left(\lambda^{-\gamma}\right)\right)\left|\Delta F_{n-1}\right|\left(\left(N\left(F_{n-1}\right)+1\right)\left|Q_{1}\right|+\sum_{\Delta \subset Q_{1}} n(\Delta)|\Delta|\right)}{2 \sqrt{\lambda} \lambda^{-s(r+i)+2 s}}$.

As $\frac{\left|h\left[x_{0}, x_{2}\right]\right|}{\left|h\left[x_{0}, x_{1}\right]\right|}<\frac{\left|x_{0}-x_{2}\right|}{\left|x_{0}-x_{1}\right|}$, we have $\frac{\left|Q_{2}\right|}{\left|Q_{1}\right|}<\frac{\left|x_{0}-x_{2}\right|}{\left|x_{0}-x_{1}\right|}\left(1+O\left(\lambda^{-\gamma}\right)\right)$. First let $Q_{2} \subset \delta_{q-2} \backslash \delta_{q}$. Then we can use (ii $\left.{ }_{n}\right)$ for $F_{n-1} \circ h\left(x_{0}, x_{1}, x_{2}\right) \subset[0,1] \backslash \delta_{(1-\alpha / s) n}$. Applying (12.27), (12.28), we obtain

$$
\frac{\sum_{\Delta C\left[x_{0}, x_{2}\right]} n(\Delta)|\Delta|}{\sum_{\Delta \subset\left[x_{0}, x_{1}\right]} n(\Delta)|\Delta|}<\frac{\left|x_{0}-x_{2}\right|}{\left|x_{0}-x_{1}\right|} \lambda^{(s-\alpha) \beta n+2 s}
$$

If $F_{n-1} \circ h\left[x_{0}, x_{2}\right]$ is not contained in $\delta_{q-2} \backslash \delta_{q}$, we have $Q_{2}=Q_{2}^{\prime} \cup Q_{2}^{\prime \prime}$ where $Q_{2}^{\prime} \subset \delta_{q-2} \backslash \delta_{q}, Q_{2}^{\prime \prime} \subset[0,1] \backslash \delta_{q-2}$. We estimate $\sum_{\Delta \subset Q_{2}^{\prime}} n(\Delta)|\Delta|$ as above, and $\sum_{\Delta \subset Q_{2}^{\prime \prime}} n(\Delta)|\Delta|$ using $\left(i_{n}\right)$ similarly to (12.19)-(12.21), and obtain

$$
\frac{\sum_{\Delta \subset\left[x_{0}, x_{2}\right]} n(\Delta)|\Delta|}{\sum_{\Delta\left[x_{0}, x_{1}\right]} n(\Delta)|\Delta|}<\frac{\left|x_{0}-x_{2}\right|}{\left|x_{0}-x_{1}\right|} \sqrt{n} \lambda^{(s-\alpha) \beta n+2 s} .
$$

For large $\lambda, \sqrt{n} \ll \lambda^{\alpha \beta n}$. Comparing with the requirement $\left(i i_{n+1}\right)$ for $k=r+i$, we obtain a sufficient condition on $r$

$$
s \beta n+2 s \leqq s \beta r .
$$

As $r>\frac{c_{0}}{2 s} n$, it suffices to have

$$
n \geqq \frac{2}{5}\left(\frac{c_{0}}{2 s}-1\right)^{-1}
$$

which holds for $s \leqq \frac{1}{13}, \beta=\frac{2}{5}, n \geqq 1$.

The account of $\Delta \subset \delta_{n}^{-M}$ gives an additional factor $\left(1+O\left(\lambda^{-\alpha n / 2}\right)\right)$ and one finishes the proof of $\left(i i_{n+1}\right)$ as above $\left(i_{n+1}\right)$.

Remark $X I I / 2$. One can check that for $n \leqq n_{0}$, when

$$
F_{n-1} \circ h\left(\lambda, \frac{1}{2}\right) \in[0,1] \backslash\left[\frac{1}{2}-\lambda^{-s / 2}, \frac{1}{2}+\lambda^{-s / 2}\right],
$$

(ii) is satisfied with $\beta=0$ [ $\lambda^{\text {skk }}$ on the right side of (ii) can be replaced by a constant]. From Remark VI/5 and (12.30) it follows that one can take $\beta$ arbitrarily small. It seems that more careful estimates should give Proposition 3 with $\beta=0$ and $k^{1+\varepsilon}$ $\left(\varepsilon>0\right.$ small) instead of $k^{3 / 2}$.

Remark $X I I / 3$. Lemma 7 implies that for any $\lambda \in \mathscr{M}$ and for $\Delta f_{k} \in \xi_{\lambda}$ so that $f_{k}: \Delta f_{k} \rightarrow[0,1], f_{k}=f_{\lambda}^{N} \mid \Delta f_{k}$,

$$
\left|D f_{k}\right|>\lambda^{c_{0} \sqrt{N} / 2}
$$


Collet and Eckmann [10] proved for a particular smooth family $f_{\delta}$ that the Liapunov exponent is positive on the trajectory of $\frac{1}{2}$ for a set of $\lambda$ of positive measure.

\section{Theorem A for a General Family. The Reduction of Theorem $B$ to Theorem A}

a) Let $f(x):[0,1] \rightarrow[0,1], f(0)=f(1)=0$, be a $C^{3}$-map, $c$ a single critical point of $f$. Consider a family $f_{\lambda}(x): x \rightarrow \lambda \cdot f(x)(\bmod 1)$. We take $\lambda$ sufficiently large and imitate the construction used for $\lambda x(1-x)$.

We take $T_{0}=(f(c))^{-1}$ so as to make $\lambda \cdot f(c)$ traverse $[0,1]$ when $\lambda$ crosses $\left[L, L+T_{0}\right]$.

Then we choose a small $\varepsilon>0$ and consider an $\varepsilon$-neighbourhood $U$ of the critical point $c$. Using the Hadamard lemma we represent $f(x)$ and its derivatives in the form

$$
\begin{aligned}
& f(x)=f(c)-a(x-c)^{2}\left(1+(x-c) \theta_{1}(x)\right) \\
& f^{\prime}(x)=-2 a(x-c)\left(1+(x-c) \theta_{2}(x)\right) \\
& f^{\prime \prime}(x)=-2 a\left(1+(x-c) \theta_{3}(x)\right)
\end{aligned}
$$

where $-2 a=f^{\prime \prime}(c)<0,\left|\theta_{i}(x)\right|<c_{1}$. Using (13.1), one can check that (4.6) with $\frac{|\Delta|}{H}$ instead of $\frac{|\Delta|}{2 H}$, and (4.7) with $\frac{|\Delta|}{a x^{2}}$ instead of $\frac{|\Delta|}{2 a x^{2}}$ are still true in $U$.

Remark $X I I I / 1$. Notice that the condition $f^{\prime \prime}(c) \neq 0$ is not necessary, $f^{(n)}(c) \neq 0$ for some $n \geqq 2$ will do as well.

Then we consider

$$
\begin{aligned}
D f_{\lambda}=\lambda f^{\prime}(x), & \frac{D^{2} f_{\lambda}}{\left(D f_{\lambda}\right)^{2}}=\frac{1}{\lambda} \frac{f^{\prime \prime}(x)}{\left(f^{\prime}(x)\right)^{2}} \\
\frac{\partial f / \partial \lambda}{\partial f / \partial x}=\frac{1}{\lambda} \frac{f(x)}{f^{\prime}(x)}, & \frac{\frac{\partial}{\partial \lambda} \frac{\partial f_{\lambda}^{-1}}{\partial z}}{\frac{\partial f_{\lambda}^{-1}}{\partial z}}=-\frac{1}{\lambda}\left(1-\frac{f^{\prime \prime} \cdot f}{\left(f^{\prime}\right)^{2}}\right) .
\end{aligned}
$$

Let

$$
A=\max _{x \in[0,1] \backslash U}\left\{\frac{1}{\left|f^{\prime \prime}(x)\right|}, 1+\frac{\left|f^{\prime \prime}(x)\right|}{\left(f^{\prime}(x)\right)^{2}}\right\} .
$$

We take $s$ from Sect. 2, and we take a $\lambda$ as a parameter. We choose $\lambda$ so large that

$$
\lambda^{s}>\max \left\{\frac{2}{\varepsilon a^{s}}, 2 A a^{1-s}\right\} .
$$

Then we choose $\delta_{1} \approx\left(c-(\lambda a)^{-s}, c+(\lambda a)^{-s}\right)$ as in Sect. 2 , and define $f_{1}(\lambda, x)$ so that $\Delta f_{1} \subseteq[0,1] \backslash \delta_{1}$. One can check that the branches $f_{1}$ and their derivatives satisfy the conditions of Step 1 with $\max _{x \in[0,1]} f(x)$ instead of $\frac{1}{4}=\max _{x \in[0,1]} x(1-x)$. Then 
for $a \lambda>N_{0}$ of Sect. 2, the inductive construction may be used, and we obtain Theorem A for the family $\lambda \cdot f(x)$.

Remark $X I I I / 2$. Theorem A holds also in the case of a family $\lambda \cdot f(x), f(0)=0=f(1)$, $f^{\prime}(0) \neq 0$, when $f(x)$ has several extremal points $c^{(1)}, c^{(2)}, \ldots, c^{(k)}$. Then the construction can be generalized in the following manner. During step $n$ we construct intervals $\delta_{n}^{(i)} \approx\left(c^{(i)}-\left(\lambda a_{i}\right)^{-s n}, c^{(i)}+\left(\lambda a_{i}\right)^{-s n}\right), 1 \leqq i \leqq k$, their preimages $\left(\delta_{n}^{(i)}\right)^{-m}$, and enlarged preimages $\left(\hat{\delta}_{n}^{(i)}\right)^{-m}$; the constants $a_{i}$ are defined according to the map $f$. The condition

$$
F_{n-1}^{(i)} \circ h^{(i)}\left(\lambda, c^{(i)}\right) \in[0,1] \bigcup_{j=1}^{k} \bigcup_{m=0}^{\infty}\left(\hat{\delta}_{n}^{(j)}\right)^{-m}
$$

defines on step $n$ the set of admissible values of the parameter $\mathscr{M}_{n}^{(i)}$, the set $\mathscr{M}$ is defined as $\mathscr{M}=\bigcap_{i=1}^{k} \bigcap_{n=1}^{\infty} \mathscr{M}_{n}^{(i)}$.

b) We reduce the proof of Theorem $B$ to the proof of Theorem $A$ using the induced map studied in [5]. Let $f_{\lambda}(x)=\lambda x(1-x), 0<\lambda \leqq 4$, and $t_{\lambda}=1-1 / \lambda$ its fixed point. We consider for $\lambda \in[4-\varepsilon, 4]$ the induced map $T_{\lambda}$ on the interval $I_{\lambda}=[1 / \lambda$, $1-1 / \lambda] . T_{\lambda}$ has $2 p$ monotone branches $T_{i \lambda}, i= \pm 1, \ldots, \pm p(p=p(\lambda))$ and one middle branch $S_{\lambda}$. Furthermore, $T_{i \lambda}=f_{\lambda}^{i+1}$ on $\Delta T_{i \lambda}$ and $S_{\lambda}=f_{\lambda}^{p+2}$ on $\Delta S_{\lambda}$. The interval $[4-\varepsilon, 4]$ is divided into a countable number of intervals $\left[\lambda_{p}, \lambda_{p+1}\right]$ such that for $\lambda \in\left[\lambda_{p}, \lambda_{p+1}\right]$ the number $p(\lambda)$ defined above is constant and as $\lambda$ passes $\lambda_{p}$, the old parabolic branch $S_{\lambda}$ breaks up into two branches $T_{\lambda}$, a new branch $S_{\lambda}$ is born, and $p(\lambda)$ grows from $p$ to $p+1$.

For some constants $c_{1}, c_{2}>0$ we have

$$
\begin{gathered}
2^{i} c_{2}<\left|\partial T_{i} / \partial x\right|<2^{i} c_{1} \quad 1 \leqq i \leqq p-1 \\
4^{p+1} c_{2}\left|x-\frac{1}{2}\right|<\left|\partial T_{p} / \partial x\right|,|\partial S / \partial x|<4^{p+1} c_{1}\left|x-\frac{1}{2}\right| .
\end{gathered}
$$

Applying (9.1) to $T_{i \lambda}$ we obtain

$$
\begin{aligned}
& \left|\frac{\partial T_{i} / \partial \lambda}{\partial T_{i} / \partial x}\right|<2^{i} c_{3} \quad 1 \leqq i \leqq p-1 \\
& \left|\frac{\partial T_{p} / \partial \lambda}{\partial T_{p} / \partial x}\right|,\left|\frac{\partial S / \partial x}{\partial S / \partial x}\right|<\frac{c_{3}}{\left|x-\frac{1}{2}\right|} .
\end{aligned}
$$

The estimate for the velocity of the top is

$$
v_{p}(\lambda)=-4^{p}\left(1+O\left(\lambda^{-p}\right)\right) .
$$

We have

$$
\begin{gathered}
\frac{\left|D^{2} T_{i \lambda}\right|}{\left|D T_{i \lambda}\right|^{2}}<c_{4} \quad 1 \leqq i \leqq p-1 \\
\frac{\left|D^{2} T_{p \lambda}\right|}{\left|D T_{p \lambda}\right|^{2}}, \frac{\left|D^{2} S_{\lambda}\right|}{\left|D S_{\lambda}\right|^{2}}<c_{4}\left(1+\frac{1}{4^{p}\left(x-\frac{1}{2}\right)^{2}}\right) .
\end{gathered}
$$


Using (11.6) we obtain for all $i$ and $z=T_{i}(\lambda, x), x \in \Delta T_{i}$

$$
\left|\frac{\frac{\partial}{\partial \lambda} \partial T_{i \lambda}^{-1} / \partial z}{\partial T_{i \lambda}^{-1} / \partial z}\right|<c_{5}\left(i+\frac{1}{\left(x-\frac{1}{2}\right)^{2}}\right) .
$$

Now we use the following property of $T_{4}$ (see [5]). There exists $d>1$ and a positive integer $q$ so that

$$
\left|D T_{4}^{q}\right|>d .
$$

Remark $X I I I / 3$. Apparently $q=1$ but it is not essential for our purpose.

For any fixed $i, T_{i}(\lambda, x)$ and its derivatives uniformly converge to $T_{i}(4, x)$ when $\lambda \rightarrow 4$. Thus for $i_{s} \in\left[0, i_{0}\right]$ and for $\lambda$ sufficiently close to 4 we still have

$$
\left|D\left(T_{\lambda i_{1}} \circ T_{\lambda i_{2}} \circ \ldots \circ T_{\lambda i_{q}}\right)\right|>d .
$$

Choose a very large $k$ and some $p \gg k$, and consider $\lambda \in\left[\lambda_{p}, \lambda_{p+1}\right]$. Let

$$
n=\left[\frac{k}{\log _{2} d}+1\right] \text {. }
$$

Let us consider consecutive compositions of the form

$$
T_{\lambda \tau_{r}}=T_{\lambda i_{1}} \circ \ldots \circ T_{\lambda i_{r}}, \quad i_{s} \in[1, k]
$$

until we have on the domain of $T_{\lambda \tau}$

$$
\left|D T_{\lambda \tau}\right|>2^{k} \text {. }
$$

Because of (13.8), for any $T_{\lambda \tau_{p}}, r \leqq q n$ (really (13.2) implies $r \ll q n$ for many $T_{\lambda \tau_{r}}$ ). Let

$$
\delta_{1}(\lambda)=\Delta S_{\lambda} \cup\left(\bigcup_{i>k} \Delta T_{\lambda i}\right), \quad J_{\lambda}=I_{\lambda} \backslash \delta_{1}(\lambda) .
$$

Then we obtain the following partition of $J_{\lambda}$.

$$
J_{\lambda}=\left(\bigcup \Delta T_{\lambda \tau}\right) \cup\left(\bigcup_{m \leqq q n} \delta_{1}^{-m}(\lambda)\right)
$$

(13.5), (13.8) and a modification of Lemma 1 imply

$$
\frac{\left|D^{2} T_{\lambda \tau_{r}}\right|}{\left|D T_{\lambda \tau_{r}}\right|^{2}}<c_{6}
$$

independent of $k$. Hence we obtain

$$
\operatorname{mes} \bigcup \delta_{1}^{-m}(\lambda)<1-\left(1-\frac{c_{7}}{2^{k}}\right)^{q n}<c_{8} \frac{k}{2^{k}} .
$$

Using (13.8), (9.1), and (11.6) we obtain

$$
\begin{gathered}
\left|\frac{\partial T_{\lambda \tau} / \partial \lambda}{\partial T_{\lambda \tau} / \partial x}\right|<2^{k} c_{9}, \\
\left|\frac{\frac{\partial}{\partial \lambda} \partial T_{\lambda \tau}^{-1} / \partial z}{\partial T_{\lambda \tau}^{-1} / \partial z}\right|<c_{10}\left(k+4^{k}\right) .
\end{gathered}
$$


Although the estimates (13.11) grow with $k$, we can choose $p$ so large that the time that the top $S_{\lambda}\left(\frac{1}{2}\right)$ spends inside the union of the enlarged domains $\bigcup \hat{\delta}_{1}^{-m}(\lambda)$ will still be proportional to its measure.

Now we are able to begin the inductive construction, with branches $T_{\lambda \tau}$ instead of $f_{1}$ and $\bigcup_{m \leq c k} \hat{\delta}_{1}^{-m}(\lambda)$ instead of $\hat{\delta}_{1}(\lambda)$. In particular, the intervals $\delta_{n}$ have the form $\delta_{n} \approx 2^{-s k(n-1)} \delta_{1}$. The estimates (13.2)-(13.10) allow the induction to continue, and if we denote by $\mathscr{M}_{p}$ the set of $\lambda \in\left[\lambda_{p}, \lambda_{p+1}\right]$ obtained by using an inductive construction similar to that in Sect. 3 , we obtain that the induced map $T_{\lambda}: I_{\lambda} \rightarrow I_{\lambda}$ has a measure $\tilde{\mu}_{\lambda}$ absolutely continuous with respect to $d x$. Besides, for some constants $c, \alpha>0$ independent of $k$ and $p$ we have

$$
\frac{\operatorname{mes} \mathscr{M}_{p}}{\left|\lambda_{p}-\lambda_{p+1}\right|}>1-c \frac{k}{\left(2^{k}\right)^{\alpha}} \text {. }
$$

The measure $\tilde{\mu}_{\lambda}$ induces an $f_{\lambda}$-invariant measure on $[0,1]$ supported on $\left[f_{\lambda}^{2}\left(\frac{1}{2}\right)\right.$, $\left.f_{\lambda}\left(\frac{1}{2}\right)\right]$. Since the time of return to $I_{\lambda}$ is finite for all $x \in I_{\lambda}, \mu_{\lambda}$ is certainly finite.

Let $\Lambda_{1}=\bigcup_{p=p_{0}}^{\infty} \mathscr{M}_{p}$. We take $k \rightarrow \infty$ together with $p$, and obtain from (13.12) that $\lambda=4$ is a Lebesgue point (from one side) of $\Lambda_{1}$. This proves Theorem $\mathrm{B}$ and the Remark of the introduction for $f_{\lambda}(x)=\lambda x(1-x) 0<\lambda \leqq 4$.

Remark XIII/4. The measures $\mu_{\lambda}$ certainly are ergodic, because the $v_{\lambda}$ are. It follows from the recent results by Ledrappier [16] that the natural extensions of $\left(f_{\lambda}, \mu_{\lambda}\right)$ are Bernoulli.

Remark $X I I I / 5$. One may conjecture that the densities $\mu_{\lambda}$ converge in $L_{1}$ to $\varrho_{4}(x)$ $=(\pi \sqrt{x(1-x)})^{-1}$, when $\lambda \rightarrow 4$. Notice that the construction always gives measures supported on the maximal possible interval $\left[f_{\lambda}^{2}\left(\frac{1}{2}\right), f_{\lambda}\left(\frac{1}{2}\right)\right]$ and thus avoids $\lambda$ corresponding to measures supported by pairwise disjoint intervals permuted by $f_{\lambda}$.

c) Consider any $f(x):[0,1] \rightarrow[0,1], f(0)=f(1)=0, f^{\prime}(c)=0$, lying in a sufficiently small $C^{3}$-neighbourhood of $x(1-x)$. Then for a family $\lambda \cdot f(x)$ there exists some $\lambda_{0}$ close to 4 so that $\lambda_{0} f(c)=1$. Considering for $\lambda \in\left[\lambda_{0}-\varepsilon, \lambda_{0}\right]$ the corresponding induced map $T_{f \lambda}: I_{f \lambda} \supset$, we obtain that $T_{f \lambda}$ has on $I_{f \lambda}$ a structure similar to the one described above for $T_{\lambda}=T_{x(1-x) \lambda}$ and (13.7) still holds for $T_{f \lambda}$. This implies Theorem B for $f_{\lambda}=\lambda \cdot f(x)$.

Now, if for some $\lambda_{0} \neq 4, f_{\lambda_{0}}=\lambda_{0} x(1-x)$ or its iteration on some interval admits the induced map described above, the construction still goes and we obtain absolutely continuous measures invariant under $f$ or under some iteration of $f$ for a set of $\lambda \in\left[\lambda_{0}-\varepsilon, \lambda_{0}\right]$ of positive measure.

One can check this is so for a countable set $\left\{\lambda_{0 n}: f \lambda_{0 n}\left(\frac{1}{2}\right)\right.$ falls into a periodic unstable orbit $\}$ and for a set $\Phi=\left\{\lambda: f_{\lambda}\left(\frac{1}{2}\right) \in K_{\lambda}=\right.$ an invariant unstable Cantor set $\}$, card $\Phi=$ continuum (see [5-7]), thus all these $\lambda$ are Lebesgue density points of $\mathscr{M}_{1}$.

Remark $X I I I / 5$. As Misiurewicz pointed out, for a family $f_{\lambda}=\lambda f(x)$ with unimodal $f(x):[0,1] \rightarrow[0,1], f(0)=f(1)=0$, having negative Schwarzian derivative, and for $\lambda_{0}$ such that $f_{\lambda_{0}}(c)$ falls into an unstable periodic orbit or an invariant unstable Cantor set, the corresponding incuded map also satisfies (13.7). Thus the same 
construction implies that for a set of $\lambda$ of positive measure $f_{\lambda}$ admits an absolutely continuous invariant measure and $\lambda_{0}$ is a Lebesgue density point of this set.

Acknowledgements. I would like to thank Ya. G. Sinai and M. Misiurewicz for many helpful discussions, M. Misiurewicz and $Z$. Nitecki for attentive reading of the text and for making many useful remarks. I am very grateful to $Z$. Nitecki for his help in preparation of the manuscript for publication.

\section{References}

1. Ulam, S., von Neumann, J.: Bull. Am. Math. Soc. 53, 1120 (1947)

2. Bunimovič, L.A.: Mat. Zametki 8, 205-216 (1970)

3. Ruelle, D. : Commun. Math. Phys. 55, 47-51 (1977)

4. Bowen, R.: Commun. Math. Phys. 69, 1-17 (1979)

5. Jakobson, M.V.: Dokl. Akad. Nauk SSSR 243, 866-869 (1978); Sov. Math. Dokl. 19, 1452-1456 (1978)

6. Misiurewicz, M.: Absolutely continuous measures for certain maps of the interval. Publ. Math. IHES (to appear)

7. Szlenk, W.: Some dynamical properties of certain dfferentiable mappings of an interval, Part I. Bol. Soc. Mat. Mexico (to appear) Part II, Ergodic theory and dynamical systems, Vol. II, Katok, A. (ed.). Boston: Birkhäuser 1981

8. Ognev, A.: Metric properties of a class of maps of the interval. Mat. Zametki (1981) (to appear)

9. Ito, S., Tanaka, S., Nakada, H.: Tokyo J. Math. 2, 221-239, 241-259 (1979)

10. Collet, P., Eckmann, J.-P.: On the abundance of aperiodic behaviour for maps of the interval. Commun. Math. Phys. 73, 115-160 (1980)

11. Guckenheimer, J.: Commun. Math. Phys. 70, 133-160 (1979)

12. Adler, R.L.: F-expansions revisited. J: Lecture Notes in Mathematics, Vol. 318, pp. 1-5. Berlin, Heidelberg, New York: Springer 1973

13. Walters, P.: Trans. Am. Math. Soc. 236, 121-153 (1978)

14. Brolin, H.: Ark. Mat. 6, 103-144 (1966)

15. Kolmogorov, A.N., Fomin, S.V.: Elements of the theory of functions and of functional analysis. Moscow, 1960

16. Ledrappier, F.: Some properties of absolutely continuous measures on an interval. Paris, Preprint, 1980

Communicated by Ya. G. Sinai

Received May 5, 1980; in revised form February 25, 1981 